\title{
Influence of Dipole-Dipole Interaction on Electron Dynamics in a Cold Rydberg Gas
}

\author{
Tao Zhou
}

B.S., Nanjing University, 2009

M.A., University of Virginia, 2012

A Dissertation presented to the Graduate Faculty of the University of Virginia in Candidacy for the Degree of Doctor of Philosophy

Department of Physics

University of Virginia

April, 2016 
CCopyright by

Tao Zhou

All Rights Reserved

April 2016 


\begin{abstract}
Electron dynamics in dipole-dipole (DD) coupled Rydberg atoms show some unique properties that do not exist in individual atoms. The goal of the research in this dissertation is to reveal and control the electron dynamics influenced by DD interaction in cold Rydberg gases.

We have studied the decay of Rydberg excitations in a cold Rb gas and find no evidence for superradiance. The decay rates and population redistribution we observe are consistent with a model that considers only spontaneous emission from, and blackbody redistribution within, isolated atoms. Suppression of superradiant emission is likely due to variations in transition energies across the cold Rydberg atom sample. For initial s states, these variations are dominated by inhomogeneities in DD exchange interactions within the random ensemble. Such inhomogeneities will necessarily be present in any measurement involving a large number of atoms where the separation between atoms is not well defined. For initial $\mathrm{p}$ states, the suppression is likely due to a combination of DD exchange and electric field inhomogeneities.

We have also explored the evolution of Rydberg wavepackets in the presence of strong dipole-dipole interactions in a frozen gas. The distribution of atom separations results in an inhomogeneity in the strength of the exchange coupling between neighboring atoms, causing a dephasing of the macroscopic coherence.
\end{abstract}

We have also explored the mechanism of wavepacket coherence transfer from one Rydberg atom to a neighboring Rydberg atom via DD interactions utilizing the resonant DD coupling transition $25 \mathrm{~s} 33 \mathrm{~s} \leftrightarrow 24 \mathrm{p} 34 \mathrm{p}$. The phase-shift of the observed interference modulations in the $34 \mathrm{p}$ signal, relative to that in $25 \mathrm{~s}$, is a signature of wavepacket coherence transfer between atoms driven by electron correlations resulting from the controlled DD coupling between them. 


\section{Acknowledgments}

I would like to thank my advisor Professor Bob Jones for his guidance and help, not only in the detailed research performance but also in scientific ethics and methodology. He taught me how to operate most of the instruments as well as helped me fix failing apparatus. His rigorous, yet optimistic, working attitude always inspires me in my research. I don't remember how many times I have felt frustrated after getting nothing from a several days' experiment. At those times, I was always motivated and energetic from his guidance, constructive criticism and sincere encouragement, after even a short talk with him. The accomplishment of this dissertation involved a lot of his help, ranging from grammar checking, to language polishing, to error correction. I really feel grateful and fortunate to have worked with him for more than 5 years.

Thanks also goes to my colleagues who have helped me in the research. Brian Richards worked with me a lot to fix failing instruments. He also programmed the data collection software. Sha Li taught me how to build setups to generate the $\mathrm{THz}$ radiation and build dye lasers. Kisra Nayomal Egodapitiya helped me adjust lasers and amplifiers many times. I also got invaluable inspiration from daily talks with them.

I am grateful for the effort from Professor Tom Gallagher and Professor Xiaochao Zheng to keep me on track in my study in the annual research review.

I would also like to thank Professor Tom Gallagher, Professor Kevin Lehmann and Professor Cass Sackett for their insights and time serving on my Ph.D dissertation defense.

The Physics Department at UVA is a fantastic place for study and research. I would like to thank everyone in this department who provided me assistance since I began to work here.

Last but not least, I would like to thank my family. It has been a while since we met. But no matter how far we are from each other, my parents' and my wife's understanding and love is always the largest support of my study and my life. 


\section{Contents}

1 Introduction ........................................................................... 1

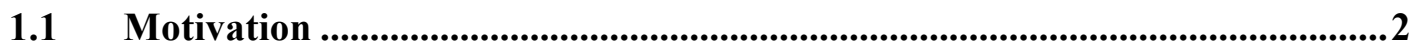

1.2 Rydberg Atoms and Dipole-Dipole Interactions.............................................3

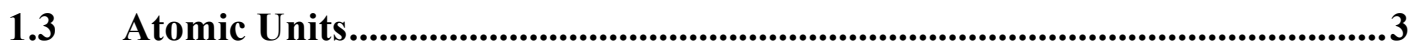

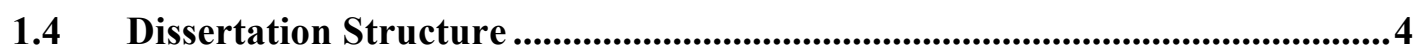

2 Experimental Setup .................................................. 8

2.1 Magneto-Optical Trap ...........................................................................................9

2.1.1 Principle of the Magneto-Optical Trap ......................................................

2.1.2 Saturated Absorption Spectroscopy …….............................................. 13

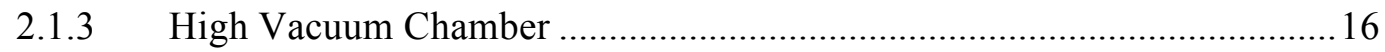

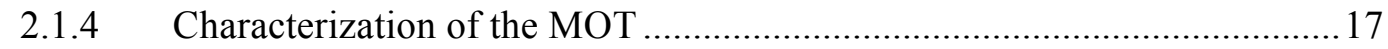

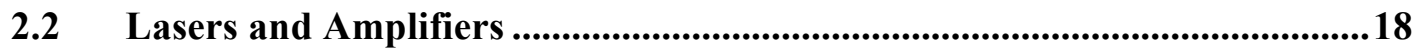

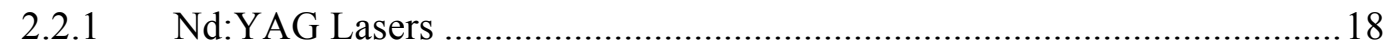

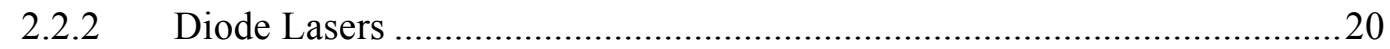

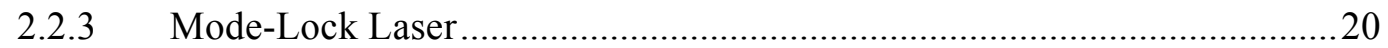

2.2.4 Chirped Pulse Amplification................................................................2

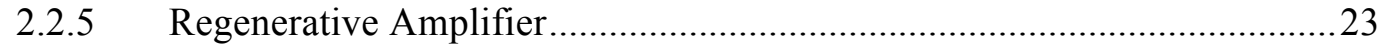

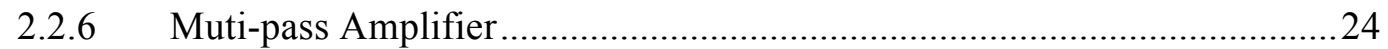

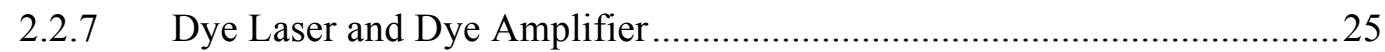

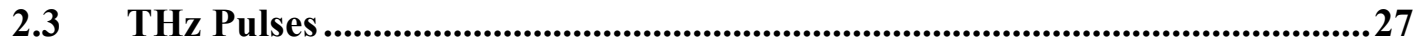

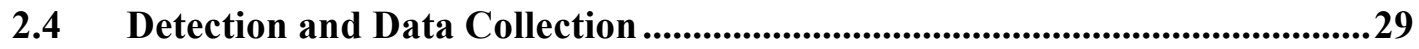

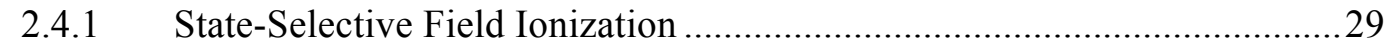

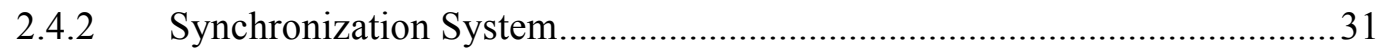

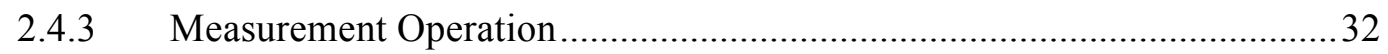


2.5 Maintenance and Daily Operation ....................................................................33

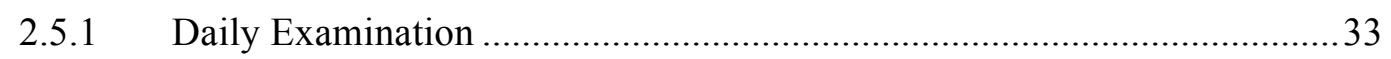

2.5.2 Operation of the Regenerative Amplifier .................................................... 34

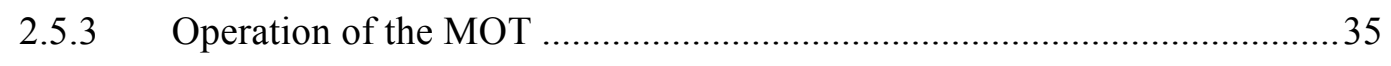

\section{Models in Simulation........................................................... 41}

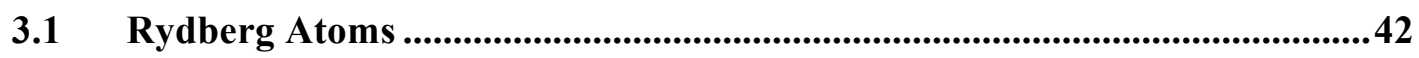

3.1.1 Modern Picture of Rydberg Atoms ..................................................... 43

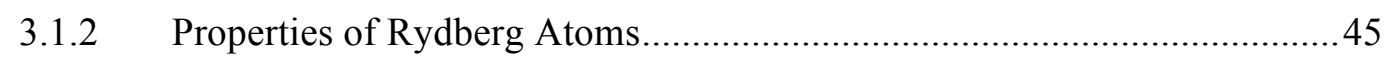

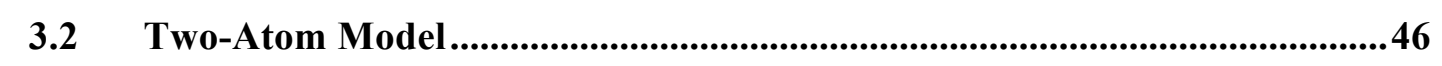

3.2.1 Introduction to the Two-Atom Model...................................................... 46

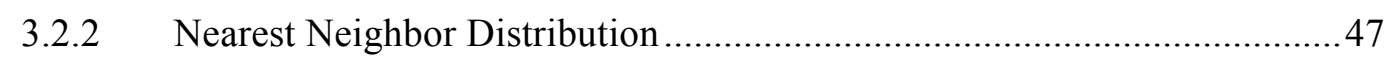

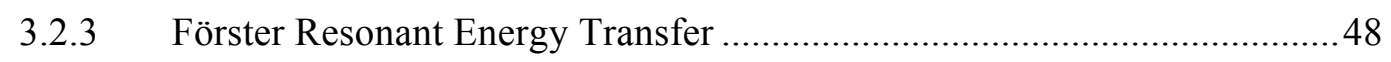

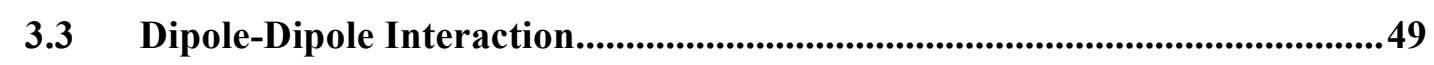

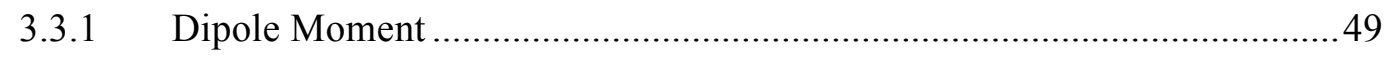

3.3.2 Dipole-Dipole Interaction in the Classical Picture ...................................50

3.3.3 Dipole-Dipole Interaction in the Quantum Picture .................................52

3.3.4 Dipole-Dipole Coupled System in an Electric Field..................................55

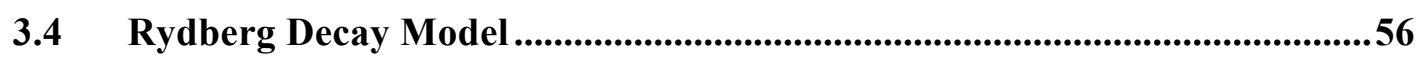

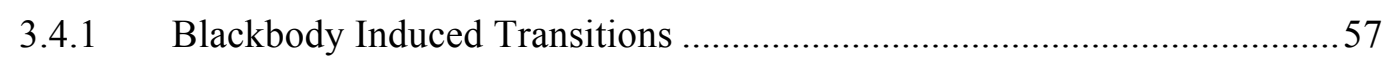

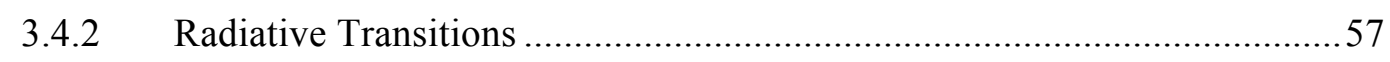

\section{Absence of Collective Decay in a Cold Rydberg Gas ..... 61}

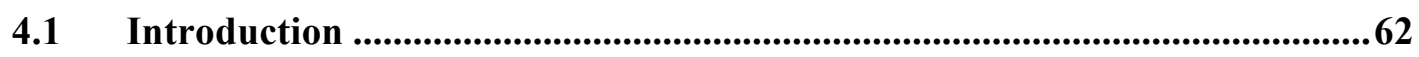

4.2 Experimental Procedure and Results ....................................................65

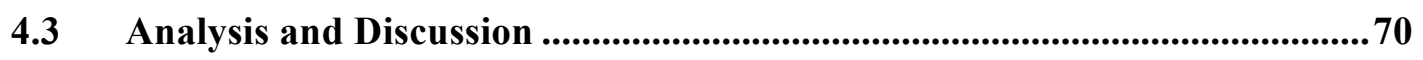

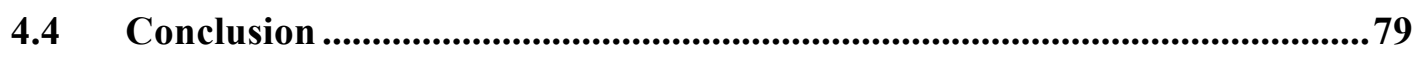




\section{Rydberg Wavepacket Evolution in A Frozen Gas of DD}

Coupled Atoms ..................................................................... 85

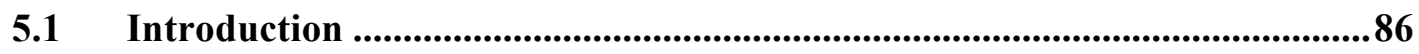

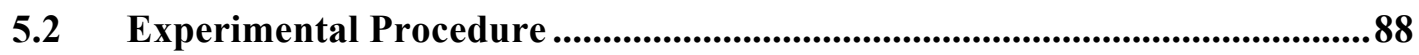

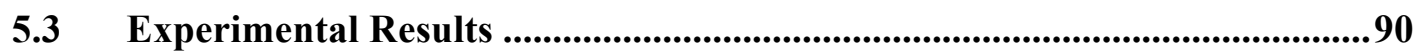

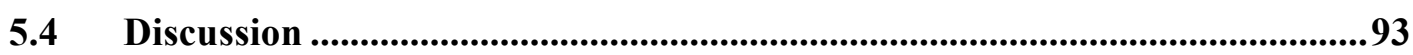

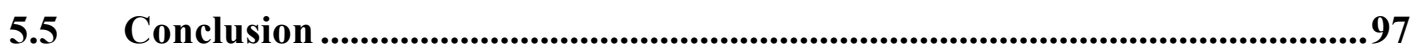

6 Wavepacket Coherence Transfer via DD interactions 102

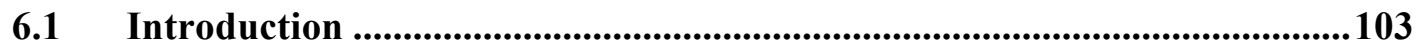

6.2 Experimental Procedure .............................................................................................107

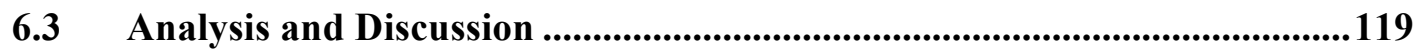

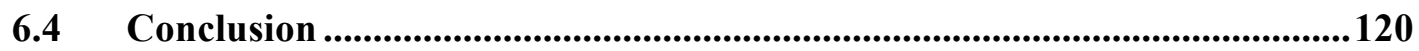

7 Summary and Future Directions ................................... 123 


\section{List of Figures}

Figure 2.1: Simplified one dimensional model for a MOT [2]. The dashed line shows the energy of the laser seen by a stationary atom.

Figure 2.2: Schematic for a MOT. It's involves a pair of anti-Helmholtz coils and six counter-propagating laser beams

Figure 2.3: Hyperfine energy structure of $85 \mathrm{Rb}$. The trap laser drives the transition, $5 \mathrm{~s} 1 / 2$ $F=3$ to $5 \mathrm{p} 3 / 2 F=4$, and the repump laser drives the transition, $5 \mathrm{~s} 1 / 2 F=2$ to $5 \mathrm{p} 3 / 2$ $F=3$.

Figure 2.4: The saturated absorption spectra for the $\mathrm{Rb} 5 \mathrm{~s} 1 / 2$ to $5 \mathrm{p} 1 / 2$ hyperfine transitions and their crossover peaks [3]. Small dark arrows identify the real resonances. The blue dotted arrow, pointing to the side of the crossover peak, indicates the point at which the trap laser is locked. The red dotted arrow indicates the position at which the repump laser is locked.

Figure 2.5: Schematic of Nd:YAG lasing [6]. It is a typical four-level lasing scheme.

Figure 2.6: Layout of Continuum Surelite Nd:YAG laser. It generates beams at the 1064 $\mathrm{nm}$ fundamental as well as the $2^{\text {nd }}$ and $3^{\text {rd }}$ harmonics [5].

Figure 2.7: Basic layout of the mode-lock laser [13]. The green line is the pump light from Millennia Vs laser and the red line is the mode-locked beam in the cavity which is centered at $780-800 \mathrm{~nm}$. The prism pair is used to compensate for group velocity dispersion (GVD). By tapping the $2^{\text {nd }}$ prism, we can produce an optical transient that develops into a stable, isolated fs pulse oscillating in the cavity.

Figure 2.8: Schematic of the chirped pulse amplification system. The seed light is first stretched using the stretcher. Then the stretched pulse gets amplified. Finally the pulse 
is again compressed to something close to its original duration, but with much greater (106-107 times) energy. 23

Figure 2.9: Schematic of the regenerative amplifier. The switch-in Pockels cell controls when a seed pulse is trapped in the resonator and the switch-out Pockels cell controls when the pulse is ejected from the cavity.

Figure 2.10: Schematic of the linear amplifier. The beam passes the Nd:YAG pumped gain medium three times and gets amplified. Pump light enters the Ti:Sapphire gain medium from two different directions to reduce the total beam flux on either surface of the crystal. 25

Figure 2.11: Schematic for a Hansch dye laser and its $2{ }^{\text {nd }}$ harmonic generation. The angle of the tuning grating determines the output frequency.

Figure 2.12: Schematic for the double cell dye amplifier used in the lab. 27

Figure 2.13: A typical THz pulse generated in the lab [9]. The duration is about 5 ps and the peak frequency is $0.2-0.4 \mathrm{THz}$. This pulse is generated using tilted-pulse-frontpumping optical rectification and the temporal profile of the pulse is characterized using the electro-optic sampling method [14]. The spectrum is then derived from the time-domain profile. 28

Figure 2.14: Schematic of the TPFP THz generation setup [9].... 29

Figure 2.15: Schematic of the tipping of atomic binding potential. The solid line is the $\sim 1 / r$ potential when there is no external field applied to the atom. The dashed line shows the potential when a field is applied to the atom. Solid strips represent energy levels. When the field is strong enough, electrons are able to escape from the trap. 30

Figure 2.16: Schematic of the synchronization system. White circles are inputs and dark circles are outputs. 32 
Figure 2.17: A typical ionization signal shown on an oscilloscope. The central peak representing the population of state $32 s$. The measurement program uses a user defined gate and integrates the area under the peak within the gate.

Figure 2.18: Front view of the chamber that houses the MOT. A, B, and C represent the three trap beams.

Figure 3.1: Classical view of Rydberg orbits of (a) $\mathrm{H}$ and (b) Na. In $\mathrm{H}$ the electron orbits around the proton. In $\mathrm{Na}$ it orbits around the +11 nuclear charge and ten inner shell electrons. In high $\ell$ states, $\mathrm{Na}$ behaves nearly identically to $\mathrm{H}$, but in low $\ell$ states the $\mathrm{Na}$ electron penetrates and polarizes the inner shell electrons of the $\mathrm{Na}+$ core [5]. 43

Figure 3.2: Schematic of atom pairs in a MOT. For each atom in the MOT, we only consider the effect of its nearest neighbor. One atom and its nearest neighbor is considered to be "a pair of atoms".

Figure 3.3: Schematic for typical FRET. Black circles represent the initial pair states and gray circles the final pair states. (a) is pp $\rightarrow$ ss', (b) ps $\rightarrow$ sp and (c) ps' $\rightarrow s^{\prime} p$ [14].

Figure 3.4: Schematic of the interaction between two classical dipoles. 51

Figure 3.5: Schematic for a DD system in an electric field. 55

Figure 3.6: Decay model for atoms starting from the $40 s$ state as an example. The red dash lines between two states indicate blackbody stimulated transitions between those two states. The blue dashed curves represent spontaneous decay. 58

Figure 4.1: (a), (c) Probabilities for finding atoms in $26 s+25 p$ (green, fastest decay), $32 s$ (red, intermediate decay), and 40s (blue, slowest decay) as a function of detection time $\tau$ for Rydberg densities of $\rho \sim 3 \times 109 \mathrm{~cm}-3$ (a) and $\rho \sim 1.5 \times 108 \mathrm{~cm}-3$ (c). Note that the sum of the $26 s$ and $25 p$ populations is shown since their corresponding features could 
not be adequately separated in the field-ionization signal. Vertical bars show the experimental data with uncertainties, and the solid curves are calculated as described in the text. Measurements and calculations for the $40 s$ decay extend to $500 \mu$ s where the remaining population is negligible. (b), (d) Probabilities for finding atoms in $26 p$ (green, fastest rise and decay), $32 p$ (red, intermediate rise and decay), and 40p (blue, slowest rise and decay) levels as a function of detection time $\tau$. The states are populated by blackbody redistribution from the initial $26 s, 32 s$, and $40 s$ levels, respectively. The data were measured simultaneously with those shown in (a) and (c). Vertical bars show the experimental data with uncertainties, and the solid curves are calculated as described in the text. The measured $p$-state probabilities are normalized to the calculations as described in the text. The calculations have no free parameters and consider only the effects of spontaneous emission and blackbody radiation on isolated atoms 68

Figure 4.2: Probabilities for finding atoms in $26 p$ (green, fastest decay), 32p (red, intermediate decay), and $40 p$ (blue, slowest decay) as functions of detection time $\tau$ for Rydberg densities of $\rho^{\sim 3} 3 \times 109 \mathrm{~cm}-3$ (a) and $\rho \sim 1.5 \times 109 \mathrm{~cm}-3$ (b). Vertical bars show the experimental data with uncertainties, and the solid curves are calculated as described in the text. Measurements and calculations for the $40 p$ decay extend to 500 $\mu$ s where the remaining population is negligible. The calculations have no free parameters and consider only the effects of spontaneous emission and blackbody radiation on isolated atoms

Figure 4.3: Measured 32p3/2 $|\mathrm{mj}|=1 / 2$ (bold line) and $|\mathrm{mj}|=3 / 2$ (thin line) excitation probabilities as a function of Rydberg laser frequency in zero applied field. The two data curves are obtained simultaneously in the same laser frequency scan. The small feature on the left (right) of the main $|\mathrm{mj}|=1 / 2(3 / 2)$ peak is the result of imperfect discrimination of the $|\mathrm{mj}|-1 / 2$ and $3 / 2$ components via SSFI. The additional peak on 
the right of the main feature in each trace is due to the trap-laser dressing of the $5 p 3 / 2$ and $5 s$ levels. Its frequency shift from the main peak reflects the Autler-Townes splitting of the $5 \mathrm{p} 3 / 2$ initial state. 76

Figure 4.4: Difference (i.e., splitting) in the transition energies for exciting 32p3/2 $|\mathrm{mj}|=$ $1 / 2,3 / 2$ from $5 \mathrm{p} 3 / 2$ as a function of applied electric field. Filled circles are measurements and the solid curve is the result of a numerical Stark map calculation assuming orthogonal "offset" and "residual" electric field components due to the MCP of 2.8 and $1.5 \mathrm{~V} / \mathrm{cm}$, respectively. The inset shows a magnified view of the portion of the main figure within the dashed window. 77

Figure 5.1: Measured population in the combined $32 s+31 p$ states as a function of the delay $\Delta t$ between two THz pulses. The left panels show data collected at low Rydberg density, $\rho \sim 3 \times 108 \mathrm{~cm}-3$, for (a) short $(\Delta t \simeq 0)$ and (b) long $(\Delta t \simeq 15 \mathrm{~ns})$ delays, respectively. The right panels show data collected at high Rydberg density, $\rho \sim 2 \times$ $109 \mathrm{~cm}-3$, for $\mathrm{c})$ short $(\Delta t \simeq 0)$ and $\mathrm{d})$ long $(\Delta t \simeq 15 \mathrm{~ns})$ delays, respectively. The decrease in oscillation amplitude at high density and long delays is apparent.

Figure 5.2: Fast Fourier transform (FFT) of the delay-dependent populations shown in Figure 5.1. 92

Figure 5.3: Measured (filled circles) and simulated (solid curve) decay ratio, $\eta$, as a function of Rydberg density.

Figure 5.4: Schematic energy level diagram for the eigenstates of a pair of two level atoms. The diagrams on the left and right sides of the figure depict the situation at large and small interatomic spacing, $R$, respectively 
Figure 6.1: Schematic energy level diagram of an atom pair. As described in previous chapters, DD interactions break the energy degeneracy and create an energy gap between the coupled pair states. 106

Figure 6.2: Calculated time dependent dipole moment along the $z$ axis for each atom in an atom pair in the presence of a resonant energy coupling $25 s 33 s \leftrightarrow 24 \mathrm{p} 1 / 234 \mathrm{p} 3 / 2$. Atom A is initially in a coherent superposition of $25 s+24 p$ (left figure) and atom B is initially in an eigenstate $33 s$ (right figure). The dipole moment on each atom has a fast oscillation (not resolved in the figure) with a period of $\sim 2.9$ ps modulated by a slow oscillation with a period of $\sim 0.8 \mu$ s, associated with the strength of the DD interaction. The distance between the two atoms is set to be $\sim 4 \mu \mathrm{m}$ the most probable nearest-neighbor separation in an ensemble at a density of $\sim 2 \times 109 \mathrm{~cm}-3$. The magnitude of the DD interaction is $\sim 7 \mathrm{MHz}$. The figures show that the wavepacket coherence travels back and forth between the atoms in one pair. 106

Figure 6.3: Magnified view of the dipole oscillation shown in Figure 6.2. Note the identical period of, but 900 phase difference between, the oscillations on the two atoms. ...107

Figure 6.4: Time line for experiment operation. The first laser pulse excites a portion of atoms from $5 p$ to $25 s$, generating atoms A. The first $\mathrm{THz}$ pulse creates a wavepacket composed of $25 \mathrm{~s}, 24 \mathrm{p}$, and $25 \mathrm{p}$ (not shown in the diagram for simplicity) on atoms A and this point could be considered as the $t=0$ point in Equation (6.3). Right after the first $\mathrm{THz}$ pulse, a second laser pulse excites another portion of atoms from $5 p$ to $33 s$, creating atoms B. After the creation of atoms B, the DD interaction enables population transfer between $25 \mathrm{~s}$ and $24 \mathrm{p}$, and 33s and 34p. The DD interaction lasts for $90 \mathrm{~ns}$, and then a second $\mathrm{THz}$ pulse, working as a probe, redistributes the population in each state. The field ionization ramp is applied as soon as practical after the $2^{\text {nd }} \mathrm{THz}$ pulse to make sure $\Delta \mathrm{T}$, which is the time interval between the total shut off of DD 
interactions and the second $\mathrm{THz}$ pulse, is set as close to 0 as possible. The ion signal collected by the MCP reveals the population in each state. A Michelson interferometer, with a variable-length delay-stage in one arm, is used to split each $790 \mathrm{~nm}$ pulse which is to generate $\mathrm{THz}$ radiation pulses, into a pump-probe pair with a delay $\sim 90 \mathrm{~ns} . .109$

Figure 6.5: Calculated average dipole moment on individual atoms for coupled via DD interactions. The plots are analogous to those shown in Figure 6.2, but a random ensemble. The density of the ensemble is $\sim 2 \times 109 \mathrm{~cm}-3$. Compared to Figure 6.3, macroscopic coherence is quickly lost because of the dephasing of the wavepackets on different atom pairs.

Figure 6.6: Measured population transfer to $34 p$ as a function of interaction time $T$ for a density $\sim 2 \times 109 \mathrm{~cm}-3$. The initial state is $(|25 s\rangle+|24 p\rangle)|33 s\rangle$. After $T \sim 200 \mathrm{~ns}$, the transfer is saturated, due to the dephasing of the DD mediated population transfer process

Figure 6.7: $34 p$ population over a short range of THz delays. Shown is the averaged result from 20 measurements. The main period of the oscillation is $\sim 2.9 \mathrm{~ns}$. The y-values do not accurately represent the modulation amplitude to DC background. In experiment, the oscillation amplitude is about $1 / 4$ of the DC background.

Figure 6.8: FFT of the delay-dependent $34 p$ population obtained with a short delay $\Delta \mathrm{T} \cong$ $10 \mathrm{~ns}$, between the $2^{\text {nd }} \mathrm{THz}$ pulse and the FI ramp. The three peaks shown in the plot (from left to right) are $9.9 \mathrm{~cm}-1(25 s->25 \mathrm{p} 3 / 2), 11.1 \mathrm{~cm}-1(25 s->24 \mathrm{p} 3 / 2)$, and 11.4 $\mathrm{cm}-1\left(25 s^{-}>24 \mathrm{p} 1 / 2\right.$ and $\left.33 s^{-}>34 \mathrm{p} 3 / 2\right)$, respectively. 114

Figure 6.9: Measured 25s population over a short range of THz delays. 116 
Figure 6.10: FFT of the delay-dependent $25 \mathrm{~s}$ population. The four peaks shown in the plot (from left to right) are $9.7 \mathrm{~cm}-1(25 s->25 \mathrm{p} 1 / 2), 9.9 \mathrm{~cm}-1(25 s->25 \mathrm{p} 3 / 2), 11.1 \mathrm{~cm}-1$ $(25 s->24 \mathrm{p} 3 / 2)$, and $11.4 \mathrm{~cm}-1(25 s->24 \mathrm{p} 1 / 2)$ respectively. 116

Figure 6.11: Phasor diagram illustrating the contributions to the detected $34 p$ signal modulations as well as the phase difference $\theta$ between the measured oscillations in $34 p$ and $25 s$. If the coherence transfer contribution is very small compared to the delayed detection associated with the $25 \mathrm{~s}$ population modulation, then the presence of coherence transfer has little effect on the magnitude of the $11.4 \mathrm{~cm}-1$ oscillation in the $34 p$ signal. However, the presence of the coherence transfer can be detected through the phase shift, $\theta$.

Figure 6.12: FFT of the time-dependent $34 p$ population at large $\Delta \mathrm{T}(>600 \mathrm{~ns})$. Peaks associated with various coherences are labeled.

Figure 6.13: Averaged $34 p$ population variation as a function of relative $\mathrm{THz}$ delays for short ( $\Delta \mathrm{T} \sim 10 \mathrm{~ns}$, red solid) and long ( $\Delta \mathrm{T} \sim 600 \mathrm{~ns}$, blue dashed) ionization delays. The signal variation at long delays $\Delta \mathrm{T}$ has been reduced by a factor of 3 to make a better comparison with the interference at small $\Delta \mathrm{T}$. The signal variation at large and small $\Delta \mathrm{T}$ are about $1 / 4$ of their DC backgrounds, repectively. 118

Figure 6.14: Calculated phase difference between the $34 p$ and $25 s$ population oscillations as a function of $\Delta \mathrm{T}$ for different amplitudes of the $2^{\text {nd }} \mathrm{THz}$ pulse. The density of the ensemble is $\sim 2 \times 109 \mathrm{~cm}-3$. V0 is the field amplitude of the $1^{\text {st }} \mathrm{THz}$ pulse, which is chosen to be $50 \mathrm{~V} / \mathrm{cm}$ to match the $25 \mathrm{~s} \rightarrow 24 \mathrm{p}$ population transfer observed in the experiment. 


\section{Introduction}

The electronic and nuclear motion degrees of freedom in Rydberg atoms are coupled by long-range dipole-dipole (DD) interactions [1]. This coupling is responsible for rich few- and many-body quantum dynamics, and its coherent manipulation can enable potential applications in the quantum control of few- and many-body systems, quantum information processing, quantum computing, etc. [2-10]. The research discussed in this dissertation focuses on the influence of DD interactions on electron dynamics within cold Rydberg atoms. This is experimentally challenging due to the large separation of time and distance scales associated with the electronic and nuclear motion, respectively. As summarized below, the results of three sub-projects are described in this dissertation. Details of the common experimental setups, computational approaches, and background knowledge are also provided. 


\subsection{Motivation}

Our group's primary research involves the exploration and manipulation of quantum dynamics in atomic and molecular systems. This dissertation describes a series of subprojects designed to further this effort. Our results provide new insight into fundamental problems in atomic physics. More than that, they may contribute to practical applications from designing many-body systems that simulate model condensed matter systems, to quantum information storage and processing, to the development of new radiation sources and detectors.

Recent work done by previous graduates Xiangdong Zhang [11] and Mary Kutteruf [12] provided much of the foundation and motivation for the work presented here. The project "Probing Time-Dependent Electron Interactions in Double Rydberg Wavepackets" done by Xiangdong Zhang helps us understand more about time-dependent electron-electron interactions within individual atoms. The project "Coherence in Rydberg Atoms: Measurement and Control" done by Mary Kutteruf explored the use of electric fields to control and measure coherence, both in electronic wavepackets and between coupled atoms in Rydberg ensembles. My project is an extension of their work but is the first to explicitly examine the impact of DD interactions between atoms on electron wavepacket dynamics within them. Another current graduate student Brian Richards is working on utilizing controlled DD interactions to manipulate the position correlation function of cold trapped atoms. Our projects are like puzzle chunks, working together to develop new capabilities and make the scientific picture more complete.

My project exploring the influence of DD interactions on Rydberg wavepackets has been divided into three sub-projects. The first one explores the role of DD interactions in suppressing collective decay ("superradiance") in an ensemble of cold Rydberg atoms (Chapter 4). The second one characterizes the role of DD interactions in dephasing the 
macroscopic coherence of an ensemble of Rydberg wavepackets (Chapter 5). The final one examines the transfer of wavepacket coherence between atoms via DD interactions (Chapter 6). These three sub-projects help us understand better how the DD interactions influence electron dynamics in an ensemble, and explore the use of controlled atom-atom coupling to induce coherent wavepacket motion within those atoms. The latter provides an effective demonstration of coherent control beyond unimolecular photo-reactions.

\subsection{Rydberg Atoms and Dipole-Dipole Interactions}

Rydberg atoms are atoms in which an electron is excited to a state with a high principal quantum number, $n \geq 10$. They are good systems for studying atom dynamics. The details about Rydberg atoms and their properties can be found in references such as [1], and are described in Chapter 3.

DD interactions can influence neighboring Rydberg atoms. Classically the interaction is the result of the electric forces between charges, electrons and the positively charged ion cores to which they are bound. When coupled by DD interactions, Rydberg atoms should not be considered as individuals but rather a system. Details of DD interactions, both from a classical view and a quantum physics perspective, can be found in Chapter 3 .

\subsection{Atomic Units}

Atomic units (au or a.u.) are commonly used in atomic physics research. For convenience, we define:

$$
\hbar=m_{e}=e=4 \pi \varepsilon_{0}=1
$$

where $\hbar$ is Planck's constant divided by $2 \pi, m_{e}$ is the mass of the electron, $-e$ is the electron charge and $\varepsilon_{0}$ is the permittivity of free space. The conversion factors between a.u. and SI units are shown in Table 1.1. 


\begin{tabular}{l|c|l}
\hline Quantity & Value in atomic units & Value in SI units \\
\hline Length & 1 & $5.2917721092(17) \times 10^{-11} \mathrm{~m}$ \\
\hline Energy & 1 & $4.35974417(75) \times 10^{-18} \mathrm{~J}$ \\
\hline Time & 1 & $2.418884326505(16) \times 10^{-17} \mathrm{~s}$ \\
\hline Velocity & 1 & $2.1876912633(73) \times 10^{6} \mathrm{~m} \cdot \mathrm{s}$ \\
\hline Force & 1 & $8.2387225(14) \times 10^{-8} \mathrm{~N}$ \\
\hline Temperature & 1 & $3.1577464(55) \times 10^{5} \mathrm{~K}$ \\
\hline Pressure & 1 & $2.9421912(19) \times 10^{13} \mathrm{~Pa}$ \\
\hline Electric field & 1 & $5.14220652(11) \times 10^{11} \mathrm{~V} \cdot \mathrm{m}^{-1}$ \\
\hline Electric potential & 1 & $2.721138505(60) \times 10^{1} \mathrm{~V}$ \\
\hline Electric dipole moment & 1 & $8.47835326(19) \times 10^{-30} \mathrm{C} \cdot \mathrm{m}$ \\
\hline Magnetic field & 1 & $2.35 \times 10^{5} \mathrm{~T}$ \\
\hline
\end{tabular}

Table 1.1: Atomic units to SI units conversion factors.

\subsection{Dissertation Structure}

Subsequent chapters describe the experimental approach, numerical simulations and several distinct projects. Each project contains both an experimental description and details on relevant simulations. Additional introductory material and common aspects of all experiments are found in chapters 2 and 3.

Chapter 2 provides the information about experimental setups. It introduces the apparatus commonly used in the experiments, as well as daily operation procedures. More details about some instruments can be found in their respective manuals and the dissertations from previous students who worked in this lab.

Chapter 3 introduces physics concepts commonly involved in the experiments, and their mathematical expression in simulations. It is not practical to include in this dissertation every single line of simulation code that was used during the research, but by 
following the models described in Chapter 3, one could reconstruct the simulations in a fairly straightforward way.

Chapter 4 describes a search for collective decay (i.e. "superradiance”) in cold Rydberg gases. We found no evidence for superradiance, despite the results reported by other groups. Our analysis suggests that, due to DD dephasing, our null result is the expected one for highly excited Rydberg atoms in the typical magneto-optical-trap geometry.

Chapter 5 describes an exploration of Rydberg wavepacket evolution in DD coupled atoms. Our results show, through experiment and simulation, that electronic wavepackets in DD coupled atoms do not evolve independently. As a result, variations in the coupling strength between pairs of atoms lead to macroscopic dephasing of the electronic wavepacket motion.

Chapter 6 describes a study of coherence transfer between wavepackets on different atoms via resonant DD interactions. We observe evidence for the development of wavepacket motion in one set of atoms resonantly driven by wavepacket evolution in neighboring atoms. We confirm the coherence transfer by measuring the relative phase between the initial and induced wavepacket oscillations, suggesting the creation of entangled atom pair states with a dynamically evolving Rydberg wavepacket on one, and only one, atom in each pair. Simulations support our interpretation.

Chapter 7 summarizes the work done in this dissertation and briefly discusses the possibilities for future experiments following those results. 


\section{Bibliography}

[1] Thomas F. Gallagher. Rydberg Atoms. Cambridge University Press (1994).

[2] M. D. Lukin, M. Fleischhauer, R. Cote, L. M. Duan, D. Jaksch, J. I. Cirac, and P. Zoller, Phys. Rev. Lett. 87, 037901 (2001).

[3] D. Tong, S. M. Farooqi, J. Stanojevic, S. Krishnan, Y. P. Zhang, R. Cote, E. E. Eyler, and P. L. Gould, Phys. Rev. Lett. 93, 063001 (2004).

[4] F. Robicheaux and J. V. Hernandez, Phys. Rev. A 72, 063403 (2005).

[5] T. Cubel Liebisch, A. Reinhard, P. R. Berman, and G. Raithel, Phys. Rev. Lett. 95, 253002 (2005).

[6] T. Vogt, M. Viteau, J. Zhao, A. Chotia, D. Comparat, and P. Pillet, Phys. Rev. Lett. 97, 083003 (2006).

[7] E. Urban, T. A. Johnson, T. Henage, L. Isenhower, D. D. Yavuz, T. G. Walker, and M. Saffman, Nat. Phys. 5, 110 (2009).

[8] Alpha Gaëtan, Yevhen Miroshnychenko, Tatjana Wilk, Amodsen Chotia, Matthieu Viteau, Daniel Comparat, Pierre Pillet, Antoine Browaeys, and Philippe Grangier, Nat. Phys. 5, 115 (2009).

[9] T. Wilk, A. Gaetan, C. Evellin, J. Wolters, Y. Miroshnychenko, P. Grangier, and A. Browaeys, Phys. Rev. Lett. 104, 010502 (2010).

[10] D. Jaksch, J.I. Cirac, P. Zoller, S.L. Rolston, R. Cote, and M.D. Lukin, Phys. Rev. Lett. 85, 2208 (2000).

[11] Xiangdong Zhang, Probing Time-Dependent Electron Interactions in Double Rydberg Wavepackets, PhD thesis, University of Virginia (2008).

[12] Mary Kutteruf, Coherence in Rydberg Atoms: Measurement and Control, $\mathrm{PhD}$ thesis, University of Virginia (2010). 
[13] A. Dalgarno, Rydberg States of Atoms and Molecules, eds. R. F. Stebbings and F.B. Dunning, Cambridge University Press (1983).

[14] H. A. Bethe and E.A. Salpeter, Quantum Mechanics of One and Two Electron Atoms, Academic Press (1957). 


\section{Experimental Setup}

This chapter describes the general experimental setup for the research discussed in this dissertation. It introduces the apparatus and procedure for state excitation, laser cooling, pulse amplification, $\mathrm{THz}$ pulse generation, data collection, etc. It also provides procedures for maintenance and daily operation. All experiments are performed on Newport RS 3000 optical tables to reduce mechanical vibrations in a temperature controlled room to reduce external thermal fluctuations. Other than specifically noted, the repetition rate of all experiments is $15 \mathrm{~Hz}$. Before beginning experiments in the lab, participants must have taken the laboratory safety training. 


\subsection{Magneto-Optical Trap}

Since its invention in 1987 [1], the Magneto-Optical Trap (MOT) has become a very important and useful tool for atomic physics research, and it is widely used to create ensembles of cold neutral atoms. The trap combines a magnetic field gradient with counterpropagating laser beams. The magnetic field gradient generates position dependent energy levels of atoms. Atoms not in the trap center preferentially absorb laser light that tends to push them back to the center.

Due to its ease of operation and relatively low cost, the MOT has been used to trap cold atoms for all the experiments described in this dissertation. We use ${ }^{85} \mathrm{Rb}$ as the atom source in all experiments. The MOT system includes a high vacuum chamber, pump and repump lasers, pressure gauges, an atom source, etc. A complete and detailed description of the system appears in Mary Kutturf's thesis [4], so only a brief version will be provided here.

\subsubsection{Principle of the Magneto-Optical Trap}

A simplified two-state model can help to understand the trapping process. As shown in Figure 2.1, suppose an atom has a ground state $J=0$ and an excited states $J=1$. Assume a weak inhomogeneous magnetic field that varies linearly with $z$ such that $B_{z}(z)=M z$ where $M$ is a constant. Due to the Zeeman effect $\Delta E=\mu m_{J} B=\mu m_{J} M z$, the field splits the degeneracy of the excited states, creating position-dependent energies for the atoms. Now assume a beam of $\sigma_{-}$circularly polarized light propagates in the $-\hat{z}$ direction and another beam with $\sigma_{+}$polarization propagates in the opposite direction. Both beams are red detuned from (i.e. on the low frequency side of) the zero-field resonance. 


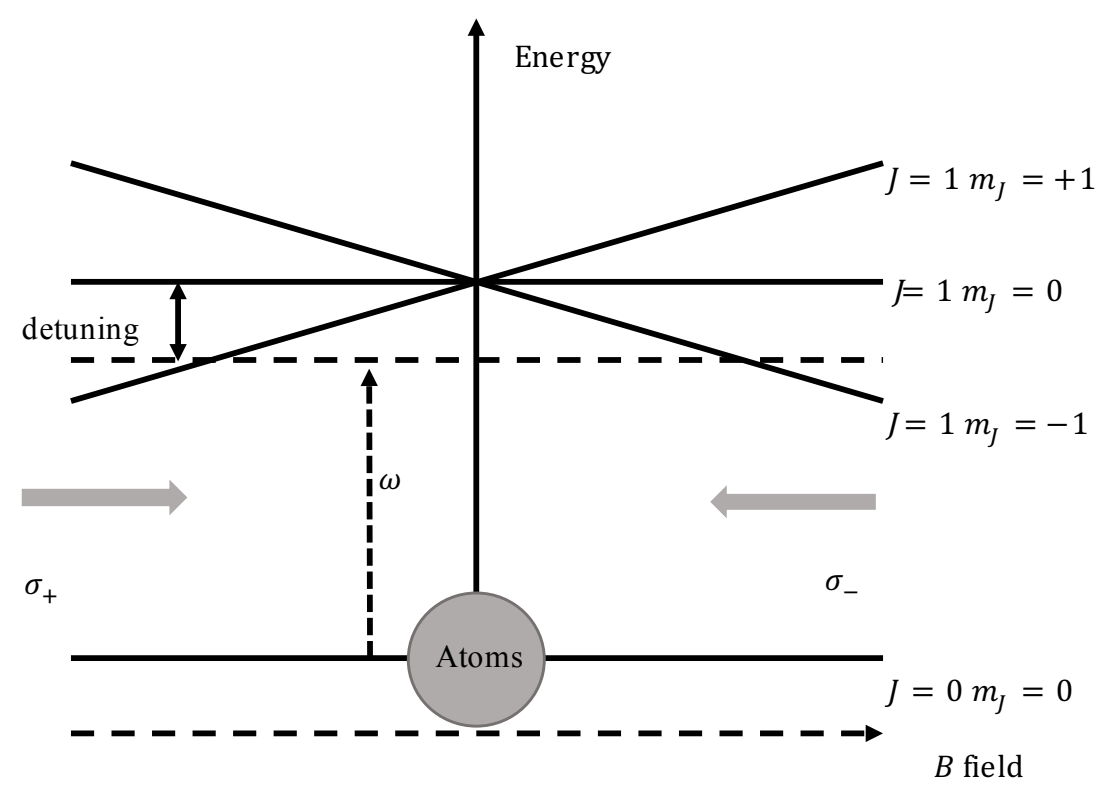

Figure 2.1: Simplified one dimensional model for a MOT [2]. The dashed line shows the energy of the laser seen by a stationary atom.

Initially, ground state atoms are located in random positions and moving in both directions with random velocities. Suppose an atom passes the center where $z=0$, with a velocity $-v$ and reaches a $-z$ position. It encounters the slightly red detuning $\sigma_{+}$beam. Due to the Doppler shift, the atom sees photons with a frequency $w+\delta$, where $w$ is the frequency of the $\sigma_{+}$beam and $\delta$ is the Doppler shift. When $w+\delta$ is close to the energy gap between the ground state and the excited $m_{J}=-1$ state, the atoms absorbs a $\sigma_{+}$photon. It later emits a photon through spontaneous decay, but the direction of the emitted photon is random. So the overall effect is that the photon gives the atom a push in $+z$ direction and this atom has been "slowed down" a little. This process repeats when the atom continues to move in the $-z$ direction and creates a damped force on the atom. More than that, for a ground atom with position $z<0$, because the energy gap between ground state and the excited $m_{J}=-1$ state is smaller than the gap between the ground state and the $m_{J}=+1$ state, it is more likely to absorb photons from $\sigma_{+}$beam and get "pushed" towards $z=0$. 
Similar rules apply to the atoms with position $z>0$. So the motion of an atom in the MOT is similar to a damped harmonic oscillator (for most cases, it is an overdamped harmonic oscillator). The atom is cooled and trapped by the MOT.

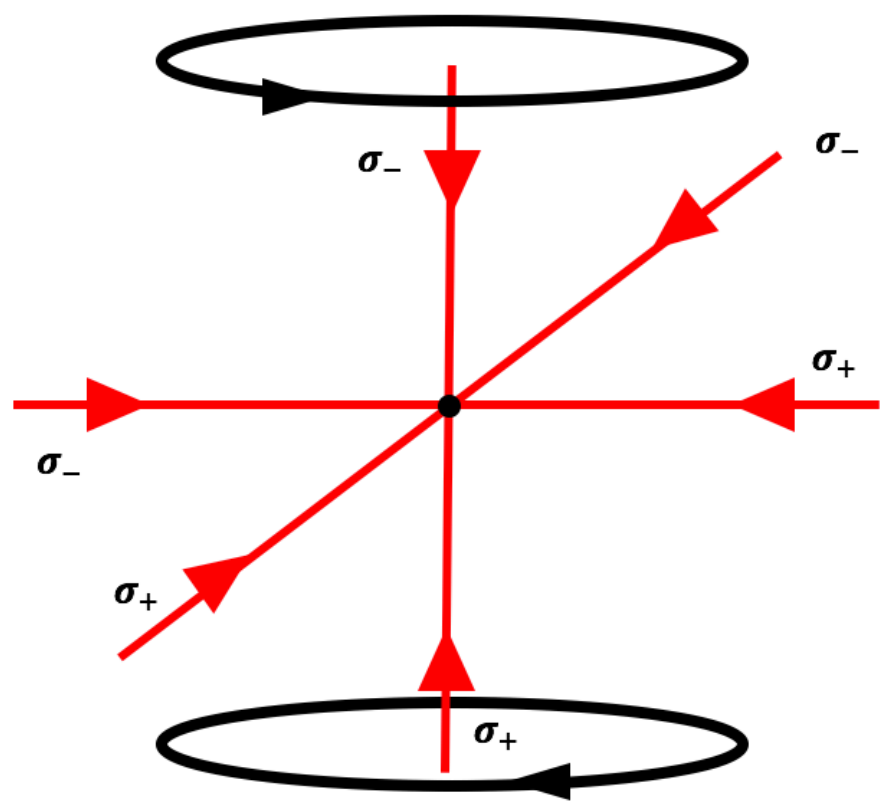

Figure 2.2: Schematic for a MOT. It's involves a pair of anti-Helmholtz coils and six counter-propagating laser beams.

As shown in Figure 2.2, a three dimensional Magneto-Optical Trap is an extension of the one dimensional model. The gradient magnetic field in a three dimensional MOT is provided by anti-Helmholtz coils. Other than the main pair of anti-Helmholtz coils shown in this schematic, there are three additional shim coil pairs which enable cancelation of Earth's magnetic field and fine tuning of the magnetic field inside the MOT. The six laser beams are derived from the same laser. We use three beam splitters to split the primary beam into three equal parts. These three beams are then reflected by mirrors to generate six counter-propagating beams in total. Before retro-reflection, the beam polarizations are rotated by waveplates placed in front of the mirrors to establish the proper $\sigma_{+}, \sigma_{-}$ orientations on each axis. 
The above discussion gives an idea about trapping two-level atoms. When dealing with ${ }^{85} \mathrm{Rb}$, there are more states involved, although the basic principal is the same. As shown in Figure 2.3, there are complicated hyperfine levels involved in the trapping of real atoms in a MOT. For ${ }^{85} \mathrm{Rb}$, the $5 s_{1 / 2}$ ground state is split into two hyperfine levels $F=2$ and $F=3$. In addition, the excited $5 p_{3 / 2}$ state is split into four hyperfine levels $F=1,2,3,4$. Ideally, the trap operates on the transfer between two of these levels, from $5 s_{1 / 2} F=3$ to $5 p_{3 / 2} F$ $=4$. But because of the small energy difference between the $5 p_{3 / 2} F=3$ and $5 p_{3 / 2} F=4$ state, the trap laser transfers a portion of atoms to $5 p_{3 / 2} F=3$. Atoms in $5 p_{3 / 2} F=3$ will quickly decay back to $5 s_{1 / 2} F=2$ and escape from the MOT. To avoid such a loss, a weak "repump" laser is introduced into the system. The repump laser transfers atoms in $5 s_{1 / 2} F$ $=2$ back to $5 p_{3 / 2} F=3$. These atoms can later decay back to $5 s_{1 / 2} F=3$, where they continue to cycle via the trap laser. 


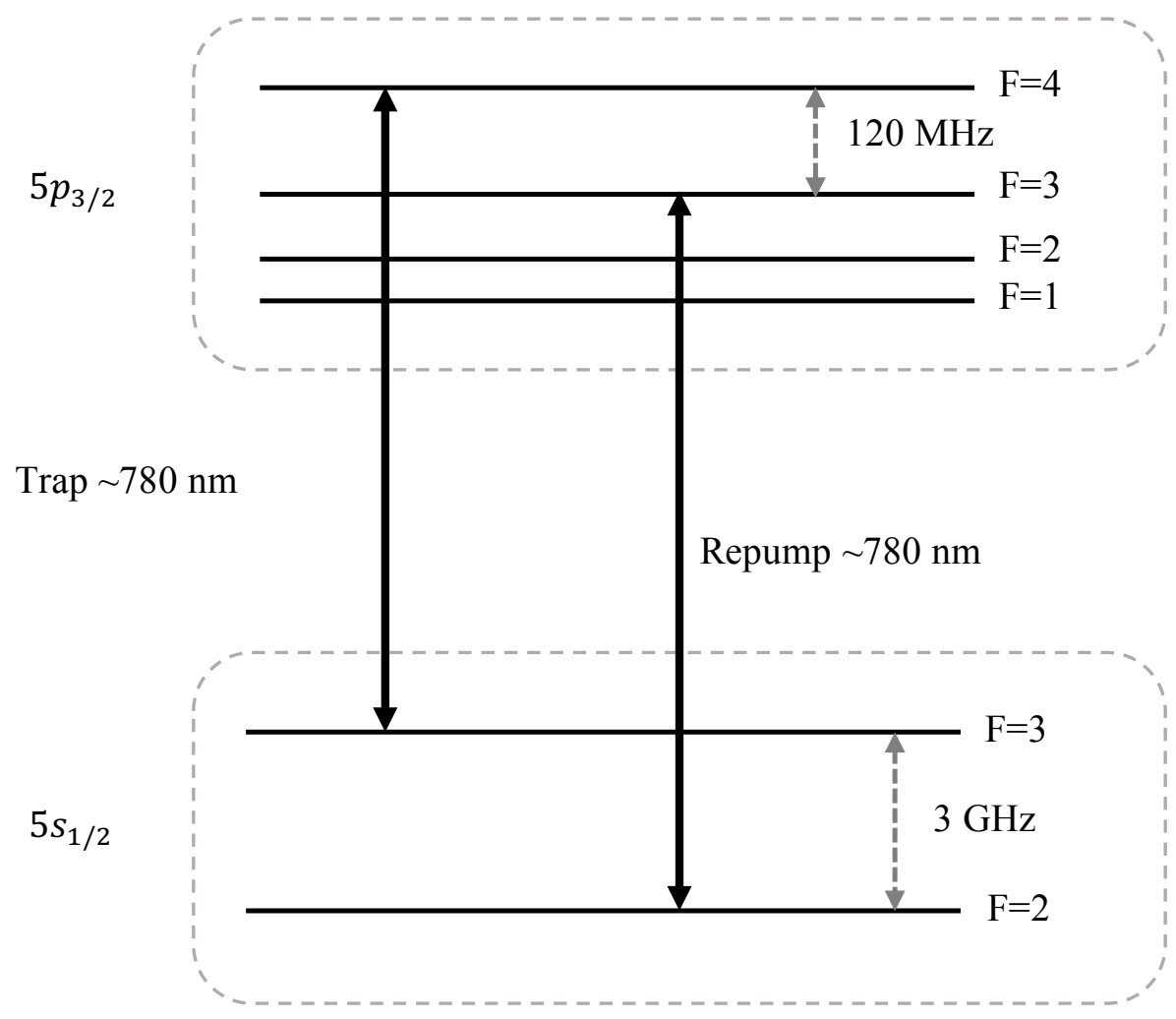

Figure 2.3: Hyperfine energy structure of ${ }^{85} \mathrm{Rb}$. The trap laser drives the transition, $5 s_{1 / 2} F=3$ to $5 p_{3 / 2} F=4$, and the repump laser drives the transition, $5 s_{1 / 2} F=2$ to $5 p_{3 / 2} F=3$.

\subsubsection{Saturated Absorption Spectroscopy}

To establish and maintain well-defined frequencies from the trap and repump lasers, the lasers are frequency locked using feedback from Saturated Absorption Spectroscopy or SAS. Our implementation of SAS is outlined below:

1. Split off a small fraction from the main beam of the laser, and direct it through a cell containing $\mathrm{Rb}$ vapor at room temperature. This beam is called the "pump" beam and it is sufficiently intense to saturate the $\mathrm{Rb}$ absorption along its path.

2. Retro-reflect the pump beam as the "probe" beam. 
3. Detect the probe intensity using a photo detector. If the beam frequency is slightly detuned from resonance, then due to the Doppler effect, different atoms in the cell moving at different velocities will absorb light from the pump and probe beams, respectively. If the laser frequency is tuned to resonance, only zero-velocity atoms can absorb photons from the pump beam. Since the pump beam saturates the absorption, there are no additional zero-velocity atoms available to absorb photons from the probe, and it passes through the cell unattenuated. Thus there is an intensity increase of the probe beam when the frequency of the beam is scanned over the resonance frequency.

In the experiment, the actual setup is a little more complicated than the above description. After passing through the cell, the beam is further split into two beams. One comes back as the probe and the other, the reference is incident on a photo-detector directly. The detected signal from the probe is then subtracted from the reference, enabling a differential measurement. In this way, we can remove any fluctuations in the laser intensity and stabilize the absorption spectrum.

The SAS signal vs trap and repump laser frequencies are shown in Figure 2.4. These signals are generated by sweeping the grating piezo voltage of the external cavity diode lasers with a triangle or sine wave. They help us lock the laser frequencies, which would otherwise drift away from resonance in a very short time.

The peaks other than the marked transition peak shown in Figure 2.4 are called “crossover" peaks. They are generated by non-zero velocity atoms when the laser frequency matches the mean frequency of each pair of true hyperfine transition peaks. We use servoloops to lock both the trap and repump lasers to the side of convenient resonances. When locked, the saturated absorption signal is compared to a constant offset voltage and the error 
signal is fed back to an amplifier which drives the piezo. This provides negative feedback to lock the lasers.

For the trap laser, the setpoint is usually placed on the side of a crossover peak, between it and the $F=3$ to $F^{\prime}=4$ resonance peak. The frequency gap between this setpoint and the $F=3$ to $F^{\prime}=4$ resonance peak is $\sim 58 \mathrm{MHz}$. In the lab, before the SAS beam has been sent to the absorption cell, it is blue detuned by $36 \mathrm{MHz}$ using an Acousto-optic modulator (AOM). So, when the trap laser has been locked, it is red detuned by $\sim 22 \mathrm{MHz}$ from the real $F=3$ to $F^{\prime}=4$ resonance. This red detuning value provides optimal cooling and trapping as determined from daily operation.

We find that the repump laser has a much higher tolerance for imperfect locking than the trap laser. This is expected because the repump is not directly responsible for cooling or trapping and influences only the small number of atoms which decay to the $F=2$ ground state. The red dotted arrow in Figure 2.4 gives the approximate setpoint frequency. The setpoint changed somewhat and there is no significant change in the MOT characteristics. 


\section{Saturated Absorption Spectra}

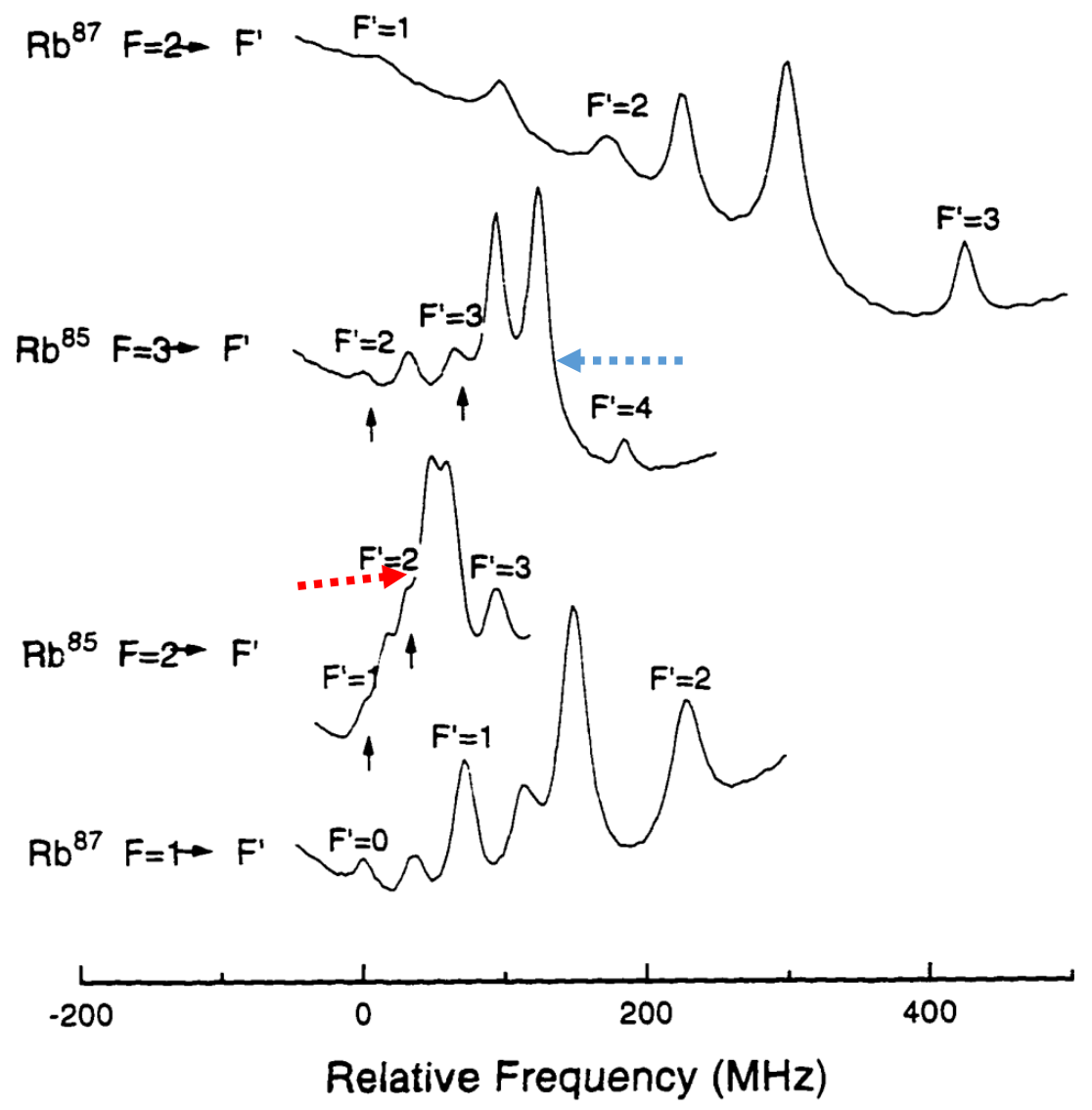

Figure 2.4: The saturated absorption spectra for the $\mathrm{Rb} 5 s_{1 / 2}$ to $5 p_{1 / 2}$ hyperfine transitions and their crossover peaks [3]. Small dark arrows identify the real resonances. The blue dotted arrow, pointing to the side of the crossover peak, indicates the point at which the trap laser is locked. The red dotted arrow indicates the position at which the repump laser is locked.

\subsubsection{High Vacuum Chamber}

At high pressures, collisions with high speed background atoms reduce the number of cold $\mathrm{Rb}$ atoms that can be collected and held in the trap. So a high vacuum environment is necessary for the MOT. In our lab, the MOT is positioned inside a high vacuum chamber, which is evacuated using an ion pump. 
The pressure in the HV chamber can be measured by two types of gauges: thermocouple and Bayard-Alpert ionization gauges, both of which are monitored by a Varian senTorr gauge. The thermocouple gauge measures pressure from $10^{-3}$ to $10^{2}$ torr. It is used during rough pumping process. The ion gauge can measure as low as $2 \times 10^{-11}$ torr. Turning it on creates a lot of ions and electrons which can affect the MOT so we don't use it during experiment. We usually refer to the approximate ion pump pressure reading for daily operation. The typical operating pressure according to the ion pump is between $10^{-9}$ torr and $10^{-8}$ torr.

\subsubsection{Characterization of the MOT}

The temperature of the atoms trapped in the MOT is $\sim 70 \mu \mathrm{K}$. Details on how the MOT's temperature is measured are provided by Mary Kutteruf in [4].

To measure the density of the MOT, we use an optical imaging method. A CCD camera detects the fluorescence from the MOT. The spatial extent and brightness of the detected signal are compared to a density calibrated signal, originally prepared by Mary Kutteruf [4]

and more recently by B. Richards. The atom density range is typically from $10^{9} \mathrm{~cm}^{-3}$ to $10^{10} \mathrm{~cm}^{-3}$ in our lab. The value of the magnetic field gradient along the coils axis is approximately 15 Gauss $/ \mathrm{cm}$, twice that in the radial direction. And the pressure reading from the ion pump is on the order of $10^{-9}$ torr. The current through the $\mathrm{Rb}$ getter source is varied to obtain the desired MOT density and atom number. 


\subsection{Lasers and Amplifiers}

\subsubsection{Nd:YAG Lasers}

Nd:YAG lasers are solid state lasers. The lasing medium is neodymium-doped yttrium aluminum garnet (Nd: $\mathrm{Y}_{3} \mathrm{Al}_{5} \mathrm{O}_{12}$ or $\mathrm{Nd}$ : $\mathrm{YAG}$ ). The medium is pumped by flash lamps and absorbs mostly in the bands between 730-760 nm and 790-820 nm [5]. It then emits light primarily centered at $1064 \mathrm{~nm}$. For our pulsed Nd:YAG lasers, a Q-switch closes the optical cavity at the optimal time to extract maximal energy in a 5-10 ns pulse from the cavity following the pulsed flashlamp excitation [refer to Figure 2.6]. The infrared output is not very useful either for directly pumping dye lasers or exciting atoms in our experiments, but it can be used to generate beams of other frequencies. For the experiments described in later chapters, Potassium Dihydrogen Phosphate (KDP) crystals are used to generate $2^{\text {nd }}$ or $3^{\text {rd }}$ harmonics of the source frequency. The $2^{\text {nd }}$ and $3^{\text {rd }}$ harmonic beams have wavelengths of $532 \mathrm{~nm}$ and $355 \mathrm{~nm}$, respectively. We use the green light at $532 \mathrm{~nm}$ from two different $\mathrm{Nd}$ :YAG lasers to pump regenerative and multi-pass Ti:Sapphire amplifiers for $\mathrm{THz}$ generation and a dye laser for Rydberg excitation. The ultraviolet light at $355 \mathrm{~nm}$ is used to pump several dye amplifiers for Rydberg excitation. 


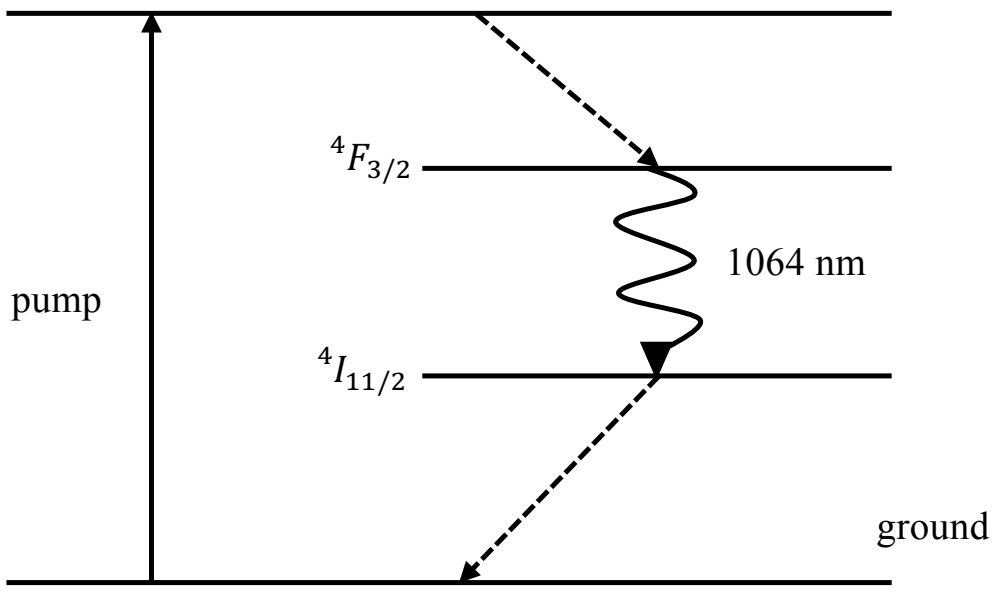

Figure 2.5: Schematic of Nd:YAG lasing [6]. It is a typical four-level lasing scheme.

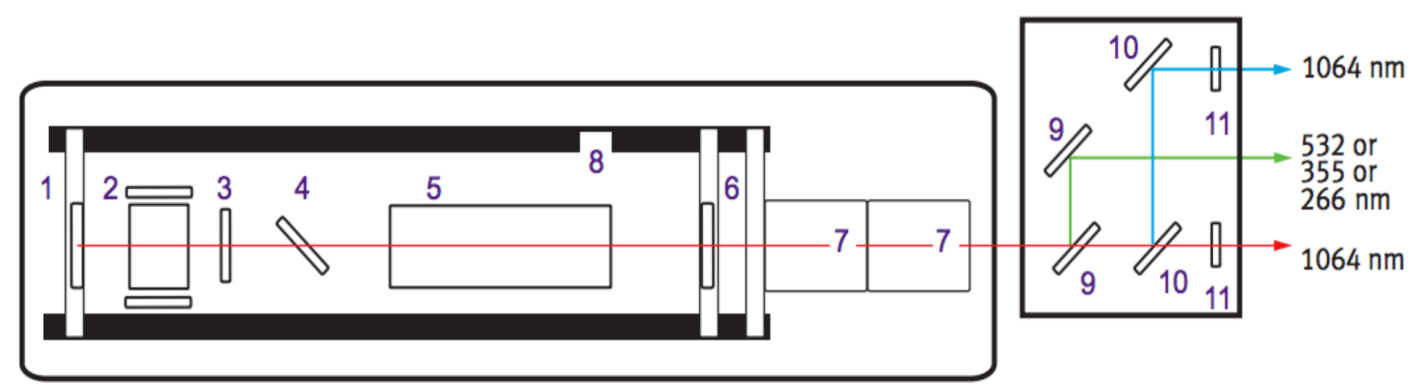

1 Rear Mirror, 2 Pockels Cell, $3 \lambda / 4$ Plate, 4 Dielectric Polarizer, 5 Oscillator Rod, 6 Gaussian Output Coupler, 7 Optional Harmonic Generators, 8 Graphite Resonator Structure, 9 Dichroic Separation, 101064 nm Mirrors, 11 Beam Block

Figure 2.6: Layout of Continuum Surelite Nd:YAG laser. It generates beams at the $1064 \mathrm{~nm}$ fundamental as well as the $2^{\text {nd }}$ and $3^{\text {rd }}$ harmonics [5].

One Nd:YAG laser combined with KDP can produce $2^{\text {nd }}$ and $3^{\text {rd }}$ harmonic pulses at the same time. But some measurements require $2^{\text {nd }}$ and $3^{\text {rd }}$ harmonic pulses at substantially different times. So two Nd:YAG lasers are used for the experiments. One is a SpectraPhysics GCR-100 Series. Its function is to generate $532 \mathrm{~nm}$ green laser light for pumping the regenerative amplifier and the multi-pass amplifier, both of which will be discussed later. The other Nd:YAG laser is a Continuum Surelite. It is used to generate ultraviolet 
$355 \mathrm{~nm}$ laser light, to pump dye lasers and dye amplifiers for the experiments. Both lasers produce pulses with durations of 5-10 ns and fire at a $15 \mathrm{~Hz}$ repetition rate.

\subsubsection{Diode Lasers}

Diode lasers are lasers use a $p-n$ junction or a $p-i-n$ structure to generate optical gain from electronic current. Semiconductor lasers are usually compact, efficient and inexpensive, so diode lasers are commonly used whenever possible. Another advantage of diode lasers is that their output frequency is tunable. The frequency of an external cavity in a diode laser can be controlled by angle tuning of a small grating in the diode laser head using a piezo. The following diode lasers are used in the experiments.

- Vortex tunable continuous wave $(\mathrm{CW})$ diode lasers from New Focus. Typical output frequency is $780 \mathrm{~nm}$ and output power $40 \mathrm{~mW}$. They are used as trap and repump lasers for the MOT.

- TA-SHG pro high power frequency-doubled tunable diode laser system from Toptica Photonics. Typical output frequency is $\sim 490 \mathrm{~nm}$ and output power $150 \mathrm{~mW}$. It is used for Rydberg excitation.

\subsubsection{Mode-Lock Laser}

The experiments also utilize pulses from a KMLabs model MTS mini Ti:Sapphire mode-locked laser. It uses passive Kerr-lens mode-locking. Its diagram is shown in Figure 2.7. The cavity is designed to have lower loss for pulsed rather than $\mathrm{CW}$ operation. The shorter the duration of the pulse in the cavity, the higher its intensity, the greater the effect of Kerr-lensing in the gain medium, and the lower the loss. The routine operation to initiate mode-locking is to displace one of the two prisms used for group velocity dispersion (GVD) compensation. The disturbance develops into a single short pulse oscillating in the cavity 
with durations as short as $15 \mathrm{fs}$ at a repetition rate of $\sim 90 \mathrm{MHz}$. The output pulse spectrum is monitored using a spectrometer. If the output is not mode-locked, it is a CW beam and the spectrum is a line with a narrow bandwidth. For well mode-locked pulses, the spectrum is very stable and has a large $(\sim 50 \mathrm{~nm})$ bandwidth. The temporal narrowness of the output pulses is more than sufficient for our experiment but the pulse energy is much too small. To generate short pulses with large enough power, we use pulses from the mode-lock laser to "seed" an amplifier. The amplification process is discussed in 2.2.4.

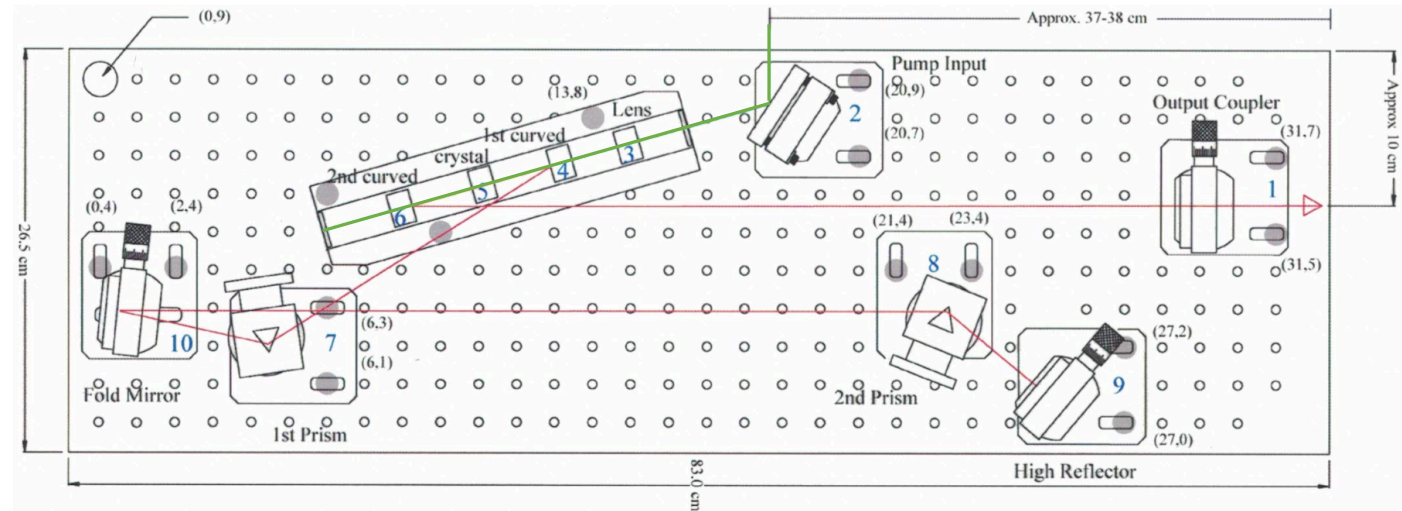

Figure 2.7: Basic layout of the mode-lock laser [13]. The green line is the pump light from Millennia Vs laser and the red line is the mode-locked beam in the cavity which is centered at $780-800 \mathrm{~nm}$. The prism pair is used to compensate for group velocity dispersion (GVD). By tapping the $2^{\text {nd }}$ prism, we can produce an optical transient that develops into a stable, isolated fs pulse oscillating in the cavity.

\subsubsection{Chirped Pulse Amplification}

As mentioned above, the output from the mode-locked laser has a very short duration but its amplitude is not large enough for the experiments. So pulses from the mode-lock laser, or the so called "seed light", has to be amplified. This is achieved through a popular technology called "Chirped Pulse Amplification". The basic idea is this:

1. Temporally stretch the 20 fs pulses to a duration of $\sim 100$ ps to reduce the peak intensity. As shown in Figure 2.8, the combination of reflecting mirrors, a grating and a lens in the stretcher acts as a pair of gratings and disperses the seed light's 
spectrum. In the stretcher, the low-frequency components of the laser pulse travel a shorter path than the high-frequency components. So after the stretcher, the pulse is temporally "chirped" and the duration is thousands of times longer than the original.

2. Amplify the stretched pulses using a regenerative amplifier and a multi-pass amplifier. Since the pulse duration is long, the peak intensity is relatively small so that it does not damage the optics.

3. Compress the amplified stretched pulses to high intensity short pulses using a compressor. The compressor acts as the inverse of the stretcher, and it also utilizes a grating. In the experiments, the compressor is adjusted to optimize the non-linear Thz generation for experiments. 


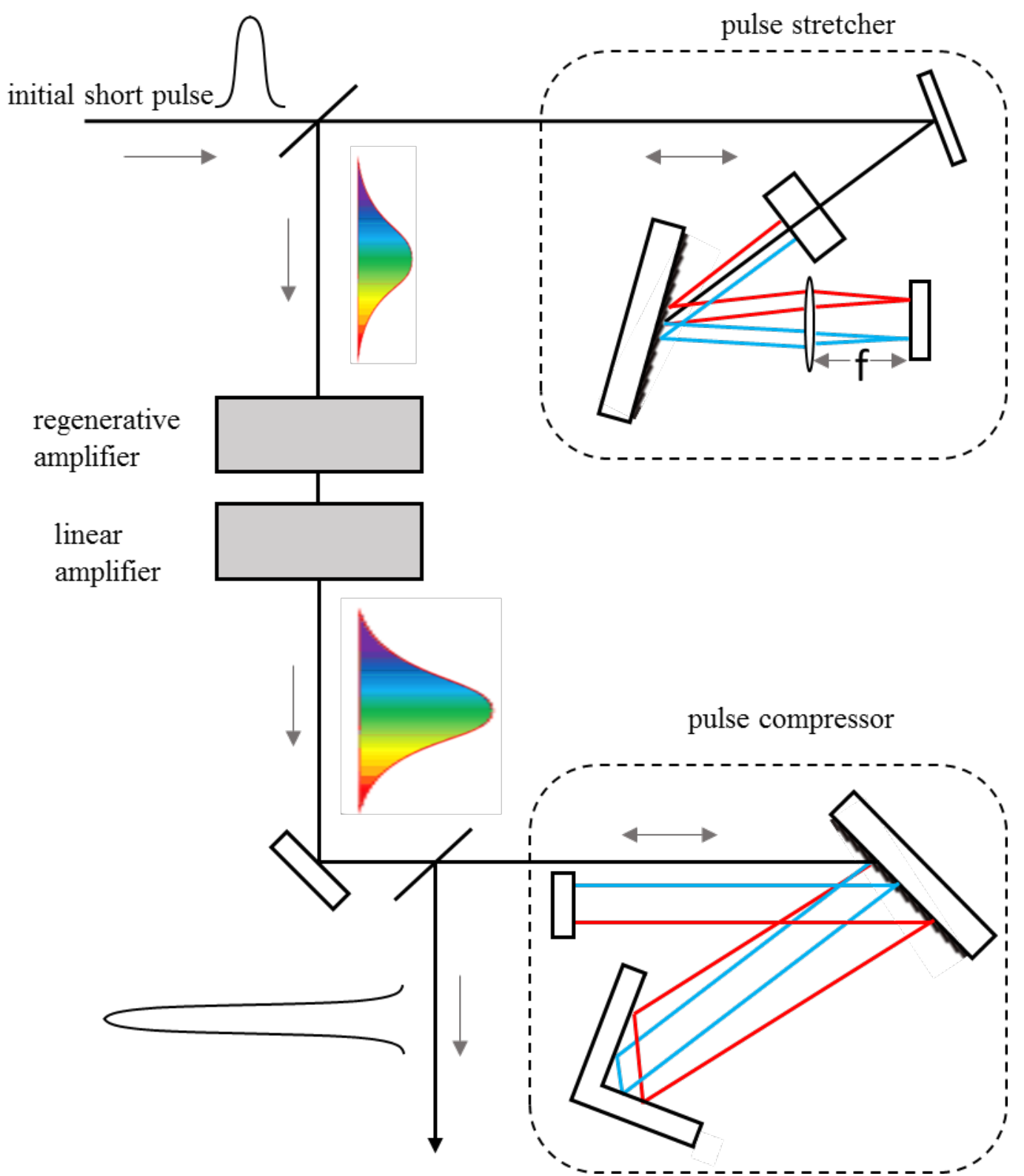

Figure 2.8: Schematic of the chirped pulse amplification system. The seed light is first stretched using the stretcher. Then the stretched pulse gets amplified. Finally the pulse is again compressed to something close to its original duration, but with much greater $\left(10^{6}-10^{7}\right.$ times $)$ energy.

\subsubsection{Regenerative Amplifier}

The first amplifier in the chirped pulse amplification is a regenerative amplifier. It uses Ti:Sapphire as the gain medium. The schematic is shown in Figure 2.9. A fast pulse "pickup" Pockels cell cooperating with a polarizer, picks a single pulse from the mode-locked pulse train before sending it into the regenerative amplifier. The input pulse has a vertical 
polarization to the paper surface and is reflected by the first polarizer to the "switch-in" Pockels cell. The switch-in Pockels cell has a high voltage applied on it. It works as a quarter waveplate and rotates the pulse's polarization from vertical to horizontal before it comes back to the first polarizer. As soon as the pulse changes the polarization to horizontal, the switch-in Pockels cell is triggered off and does not affect the pulse's polarization during the pulse's journey in the cavity. Now the switch-in Pockels cell also prevents any small amplitude leakage pulses from the pick-up Pockels cell from entering the cavity. The switched-in pulse, with horizontal polarization, goes through the first polarizer and passes through the gain medium where it is amplified. It then goes through the switch-out Pockels cell, which is off, and is then reflected back to the cavity. After multiple runs (usually 20 runs) in the cavity to gain maximum intensity, the switch-out Pockels cell is triggered on, and the pulse is switched out with a vertical polarization.

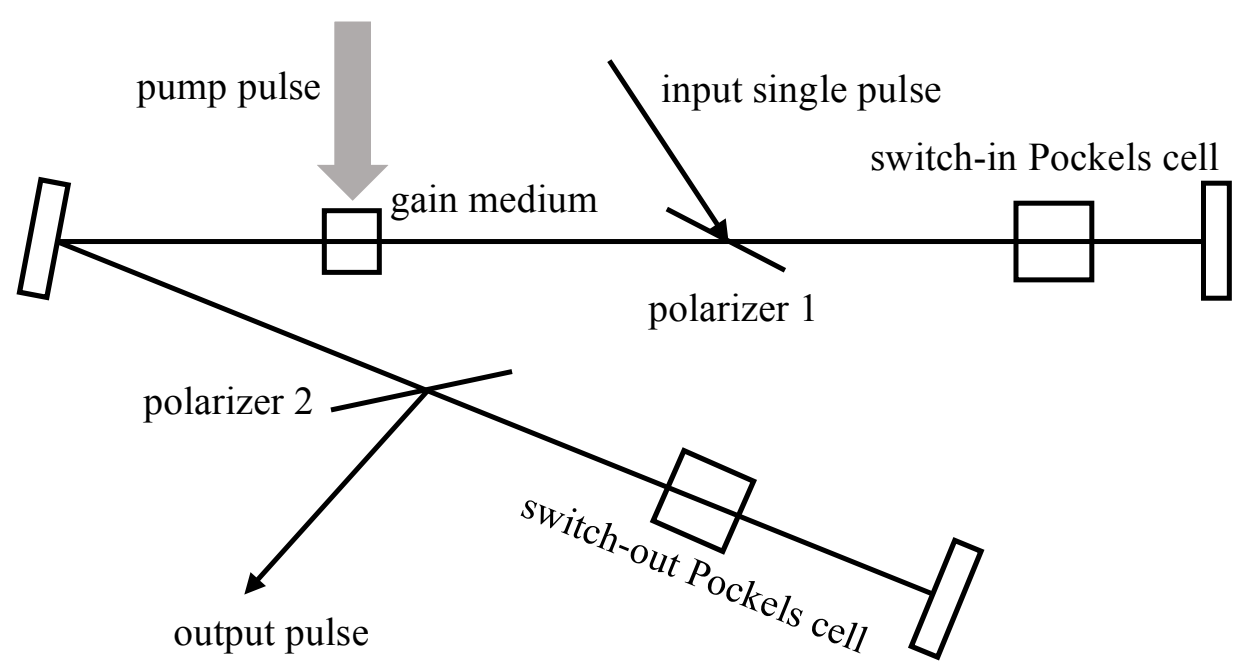

Figure 2.9: Schematic of the regenerative amplifier. The switch-in Pockels cell controls when a seed pulse is trapped in the resonator and the switch-out Pockels cell controls when the pulse is ejected from the cavity.

\subsubsection{Muti-pass Amplifier}

An additional multi-pass or "linear" amplifier is used when the pulse energy from the regenerative amplifier $(\approx 3 \mathrm{~mJ})$ is not large enough for the experiments. The pulse obtains 
additional amplification by making three passes through another Nd:YAG laser pumped Ti:Sapphire crystal. The highest output from the linear amplifier in our lab is over $40 \mathrm{~mJ}$ (prior to the compressor). It is shown schematically in Figure 2.10.

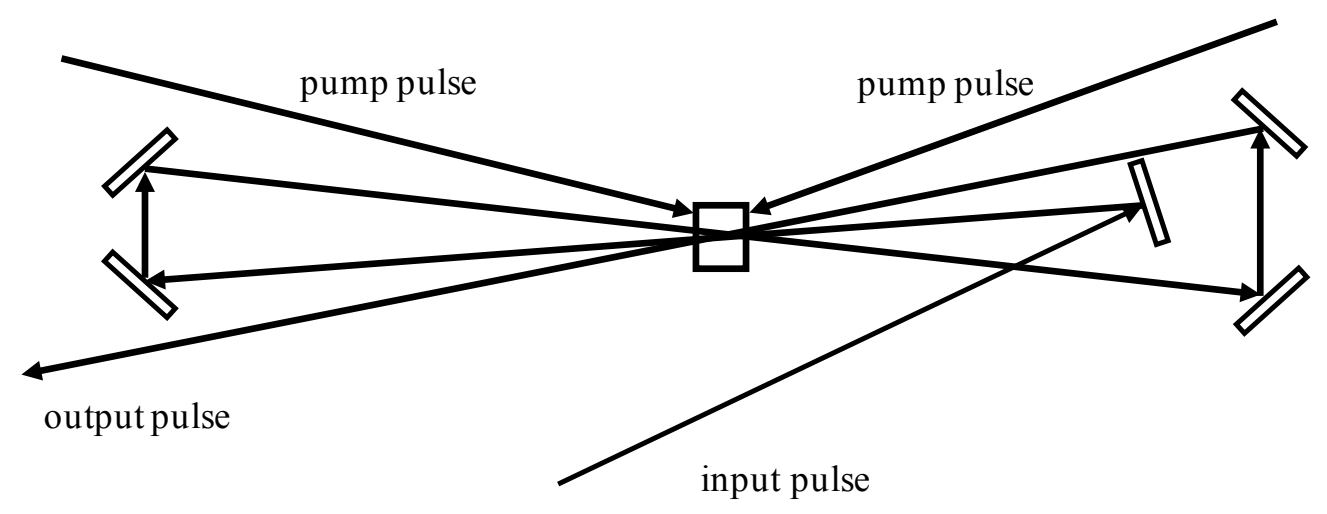

Figure 2.10: Schematic of the linear amplifier. The beam passes the Nd:YAG pumped gain medium three times and gets amplified. Pump light enters the Ti:Sapphire gain medium from two different directions to reduce the total beam flux on either surface of the crystal.

\subsubsection{Dye Laser and Dye Amplifier}

In our experiments, a Hansch-style dye laser [7] has been used for Rydberg excitations. This dye laser is used to generate $25 s$ Rydberg atoms. The proper laser dye is LDS 925, which is dissolved in methanol solvent, with a concentration of $250 \mathrm{mg} / \mathrm{L}$. This solution is pumped by the $2^{\text {nd }}$ harmonic from Continuum Surelite. The pump light has been focused about a millimeter into the dye cell by a cylindrical lens, creating a line of gain medium across the face of the cell. The dye cell works as a fluorescence generator, as well as an amplifier. The telescope expands and collimates the beam to illuminate more grating lines for narrower line width. The grating is rotatable, which determines the frequency of the light diffracted back to the cavity. 


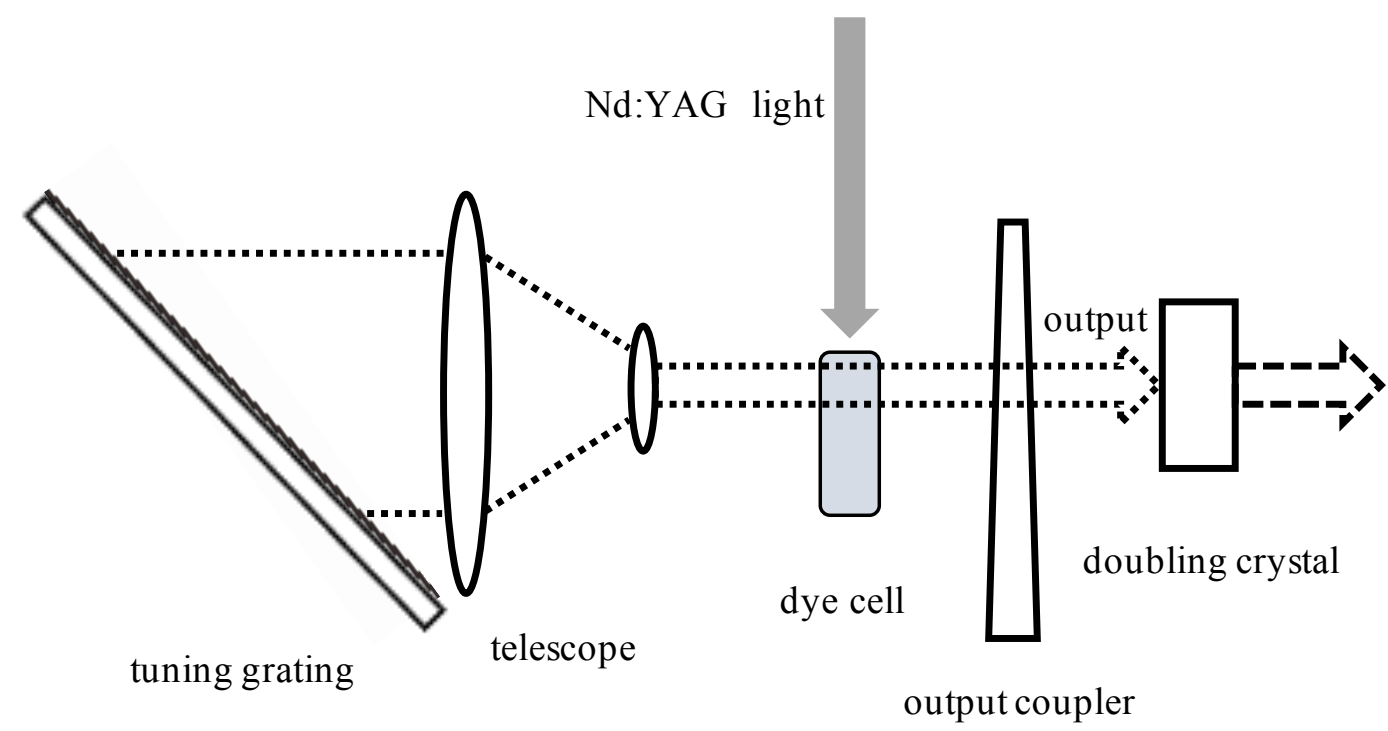

Figure 2.11: Schematic for a Hansch dye laser and its $2{ }^{\text {nd }}$ harmonic generation. The angle of the tuning grating determines the output frequency.

The infrared $(\sim 970 \mathrm{~nm})$ output of this laser is then frequency doubled to generate blue laser pulses at $486 \mathrm{~nm}$. The spectrum of the output beam is the convolution of the input spectrum with itself. With a $486 \mathrm{~nm}$ dye laser, on some shots there is no light of the proper transition frequency. Compared to that, spikes in the original dye laser spectrum are suppressed and broadened, and gaps between these spikes are partially filled in. When sent through a saturated amplifier, the gaps between spikes are further filled in ensuring that there is always some light of the Rydberg excitation frequency. An etalon ( $1 \mathrm{~mm}$ thickness) is put after the doubling crystal and changing its angle fine tunes the output frequency, before the beam is sent to the saturated amplifier. The amplified beam is then sent to the chamber, and drives $\mathrm{Rb}$ atoms from $5 p$ state to $25 s$ state.

A flowing dye cell without cavities can serve as an amplifier as shown in Figure 2.12. The double amplifier shown is used to amplify laser pulses from dye or diode lasers. Before performing measurements, we ensure that the Rydberg transition has been saturated by the amplified beams. If there is no significant reduction of the Rydberg population on a state 
(e.g, $25 s$ ) when an $80 \%$ transmission filter is inserted in the beam path, we are confident that the transition is saturated.

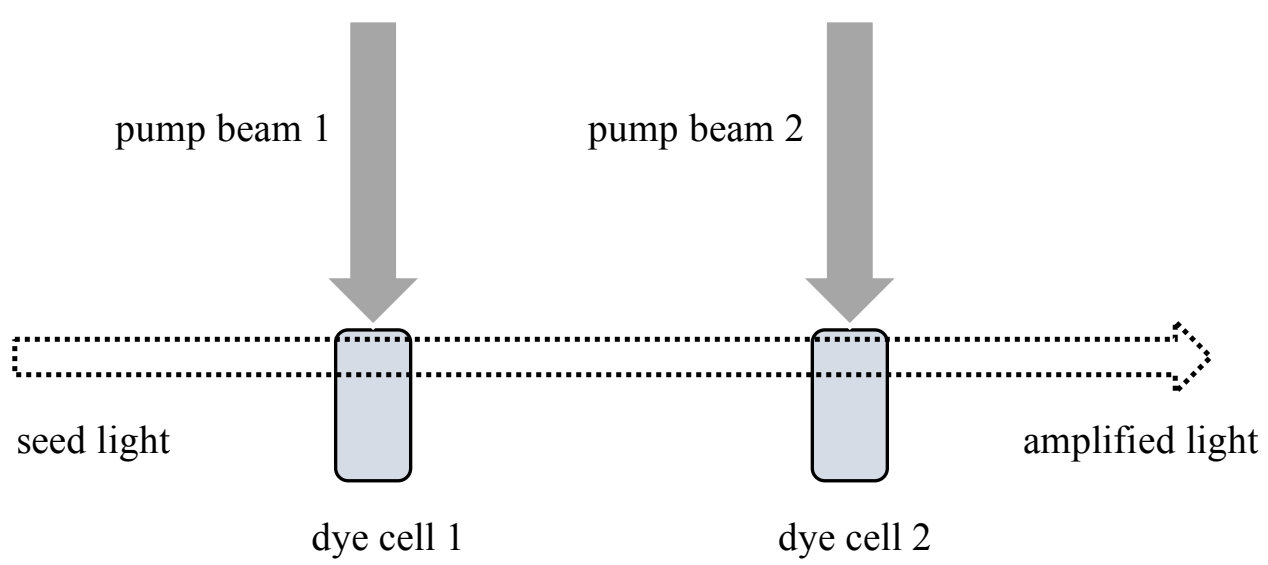

Figure 2.12: Schematic for the double cell dye amplifier used in the lab.

\section{$2.3 \mathrm{THz}$ Pulses}

The duration of the THz pulses generated in our lab is on the order of a few ps (a typical $\mathrm{THz}$ pulse generated in the lab is shown in Figure 2.13). The THz pulses can coherently redistribute Rydberg population on time scales that are negligible compared to the relatively long time required for other interactions (such as Dipole-Dipole interactions). So we use THz pulses to induce and probe coherent population distribution in the experiments described in chapters 5 and 6 . The THz setup for experiments in this dissertation follows that developed by Sha Li for her research. For a detailed discussion of THz generation and characterization, one can refer to her dissertation [9]. A brief introduction of $\mathrm{THz}$ generation will be provided in this chapter. 


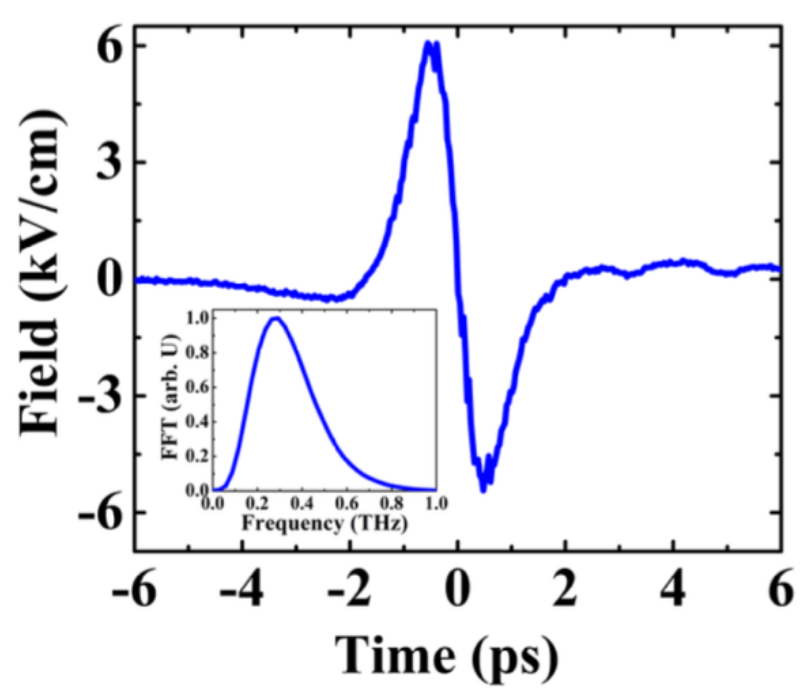

Figure 2.13: A typical THz pulse generated in the lab [9]. The duration is about $5 \mathrm{ps}$ and the peak frequency is $0.2-0.4 \mathrm{THz}$. This pulse is generated using tilted-pulsefront-pumping optical rectification and the temporal profile of the pulse is characterized using the electro-optic sampling method [14]. The spectrum is then derived from the time-domain profile.

The THz pulses used in the experiments are generated via tilted-pulse-front-pumping (TPFP) optical rectification of femtosecond laser pulses in a $\mathrm{LiNbO}_{3}$ crystal [8]. It utilizes the so called difference frequency generation (DFG) phenomenon and is actually a cascaded intrapulse DFG [10]. When an ultrafast laser pulse (broad bandwidth) passes through a nonlinear crystal with non-vanishing $\chi_{2}$, it creates a polarization that follows the intensity envelope of the pump field. The induced polarization is a source of electromagnetic radiation. The frequency bandwidth of our femtosecond pulses lies in $\mathrm{THz}$ regime (e.g. a sinusoidal pulse with Gaussian envelope, $\Delta \tau_{F W H M}=100 \mathrm{fs}$ has a bandwidth of $\Delta f_{F W H M}=4.41 \mathrm{THz}$ ), thus optical rectification of ultrafast femtosecond laser pulses can be used to generate $\mathrm{THz}$ radiation.

Efficient nonlinear conversion requires phase matching. Hebling et al. proposed a tilted-pulse-front-pumping scheme for phase matching [11]. In the TPFP scheme, the intensity front of the near-infrared (NIR) pump pulse is tilted by an angle of $\gamma$ inside the crystal via grating diffraction [8], and the crystal is cut at this angle to obtain a beam exiting 
normal to the crystal surface. As shown in Figure 2.14, when $v_{N I R}^{g r} \times \cos \gamma=v_{T H z}$, where $v_{N I R}^{g r}$ is the group velocity of near-infrared light beam in the crystal, the propagation direction of the generated $\mathrm{THz}$ radiation is perpendicular to the intensity front of the pump, and normal to the exit face of the prism shaped crystal.

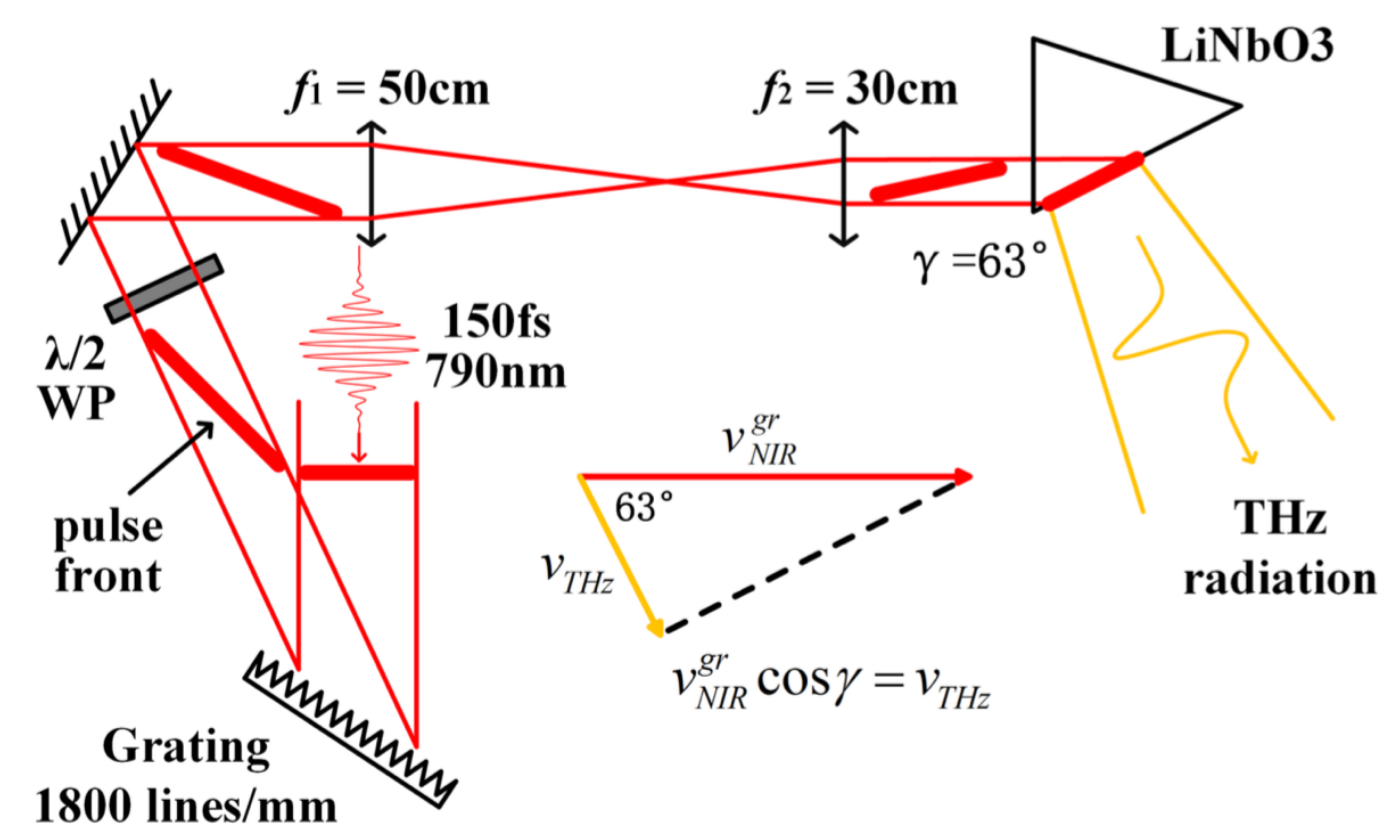

Figure 2.14: Schematic of the TPFP THz generation setup [9].

\subsection{Detection and Data Collection}

\subsubsection{State-Selective Field Ionization}

As an efficient state distribution detection technology, State-Selective Field Ionization (SSFI) has been used widely to measure the state distribution of atoms [12].

In alkali atoms, the outermost electron is bound in a potential trap that at large distances $r$, has the form, $V(r) \sim 1 / r$. When a static field is applied to the atom, the potential tips to one side as shown in Figure 2.15. This lowers the barrier that traps the electron. When the barrier is low enough, the electron is able to escape from the potential trap. In a rising field 
ramp, higher energy electrons tend to be ionized sooner than low energy electrons, so that the probability that the atom was initially in a state with a particular principal, and (perhaps) angular momentum quantum number, is proportional to the ionization signal at a particular time.

In the MOT chamber, there are four metal rods. Two of them are connected to a high voltage pulse supply and the other two are connected to ground or low static voltage. Pulsed voltages applied to these 4 rods create strong electric fields with $1 \mu$ s (slow ionization field) or $500 \mathrm{~ns}$ (fast ionization field which rises to half maximum in only $100 \mathrm{~ns}$ ) rise time to reach maximum. Atoms in the field will be ionized and the ions will fly in the ionization field toward a detector composed of micro-channel plates (MCP). Atoms in different states are ionized at different times, so the population in different states can be distinguished in the time dependent current from the MCP detector.

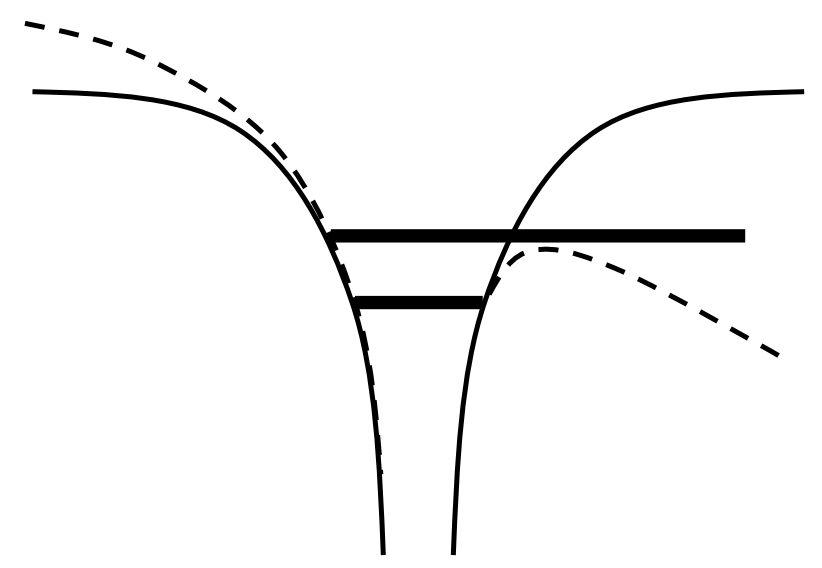

Figure 2.15: Schematic of the tipping of atomic binding potential. The solid line is the $\sim 1 / r$ potential when there is no external field applied to the atom. The dashed line shows the potential when a field is applied to the atom. Solid strips represent energy levels. When the field is strong enough, electrons are able to escape from the trap. 


\subsubsection{Synchronization System}

Before making any measurements, the lasers and electric field pulses must be synchronized. The synchronization is controlled by a timing system in the lab. It introduces a combination of clocks, delay generators and synchronization boxes. The system is flexible enough to readily adapt to changes in the experimental approach. Figure 2.16 shows the synchronization system working in the experiment described in Chapter 6. Small changes are needed for other experiments. The "master" clock is a divider which divides the commercial $60 \mathrm{~Hz}$ electrical supply by 4 and provides a $15 \mathrm{~Hz}$ clock to trigger a multichannel digital delay/pulse generator, Model DG535 from Stanford Research Systems. One channel of this delay generator triggers a second DG535 which controls the firing of the lamps inside the Surelite Nd:YAG laser. Another channel triggers the lamp of GCR-100 Nd:YAG laser. The GCR-100 provides a "ready" signal at the optimal time for firing its Qswitch. This Q-switch request signal is delayed by up to one period of the mode-locked pulse train from the Ti:Sapphire oscillator in the SM-1 synchronization box. The SM-1 output is synchronized with the mode-locked pulse train and triggers the GCR-100 Qswitch as well as two additional digital delay generators (a DG535 and a DG645). The DG535 controls the Pockels cells in the regenerative amplifier. The DG645 controls the Qswitch of the Surelite Nd:YAG, and triggers the ionization field, oscilloscopes, etc. The delays are easy to change on these delay generators so this system can handle different timing for different experiments. 


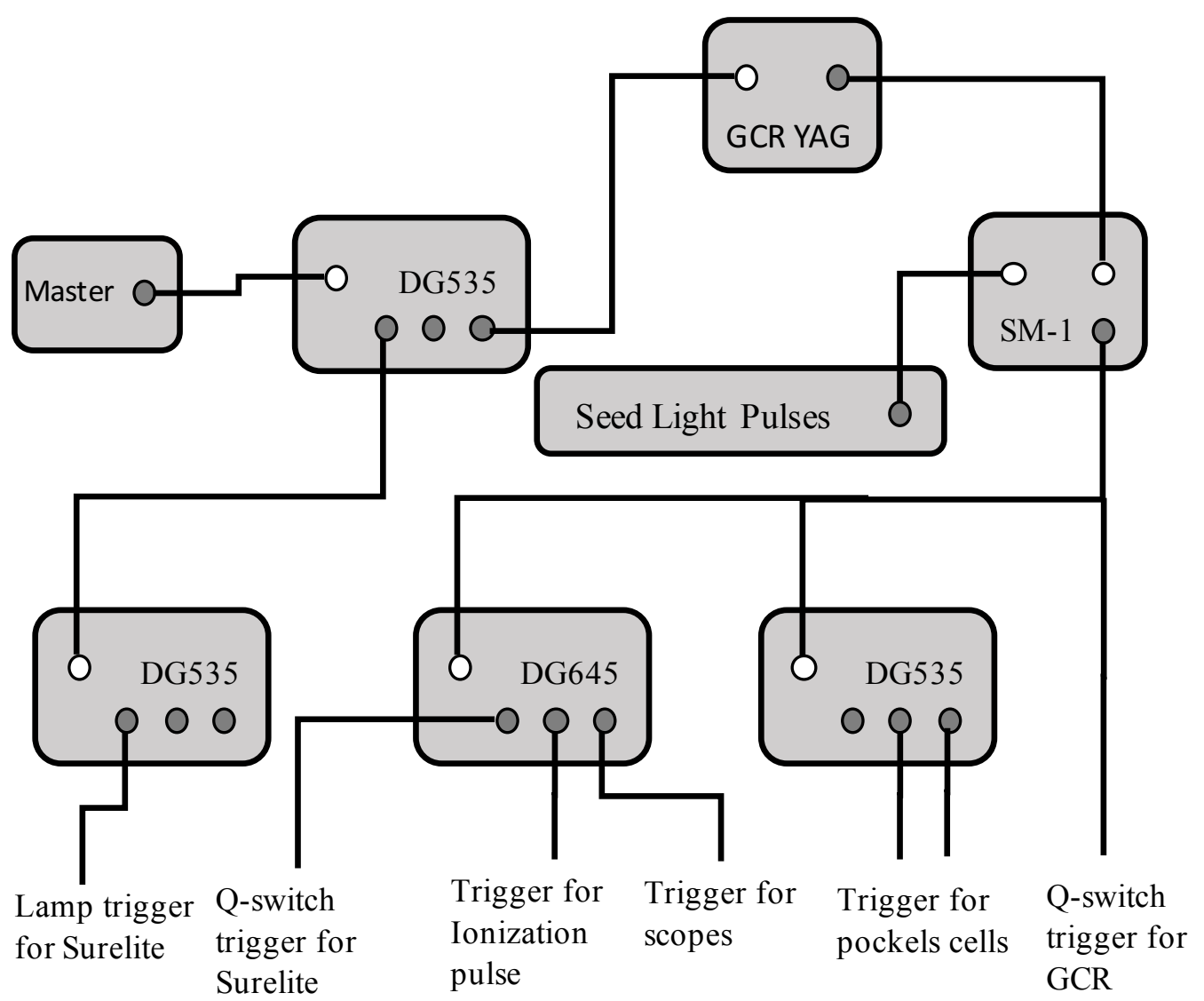

Figure 2.16: Schematic of the synchronization system. White circles are inputs and dark circles are outputs.

\subsubsection{Measurement Operation}

The time-dependent ionization signal from the MCP detector is collected using oscilloscopes, and the oscilloscopes transfer the data to computers via data collection programs written in Labview. A typical electric signal representing a state population is a peak with some temporal width. Usually, the larger a state's population is, the higher the peak is. But the height alone is not an accurate measure of the population. Instead, the area of peak is proportional to the state population. As shown in Figure 2.17, the peak within the temporal gate or window is the ionization signal associated with a particular state. Using the Labview code, we can record, on each laser shot, the area under the peak in the user defined gate. After subtracting this signal area by the background area recorded when there 
is no ionization, we obtain the Rydberg population. As the population changes, the integrated value in the gate changes accordingly.

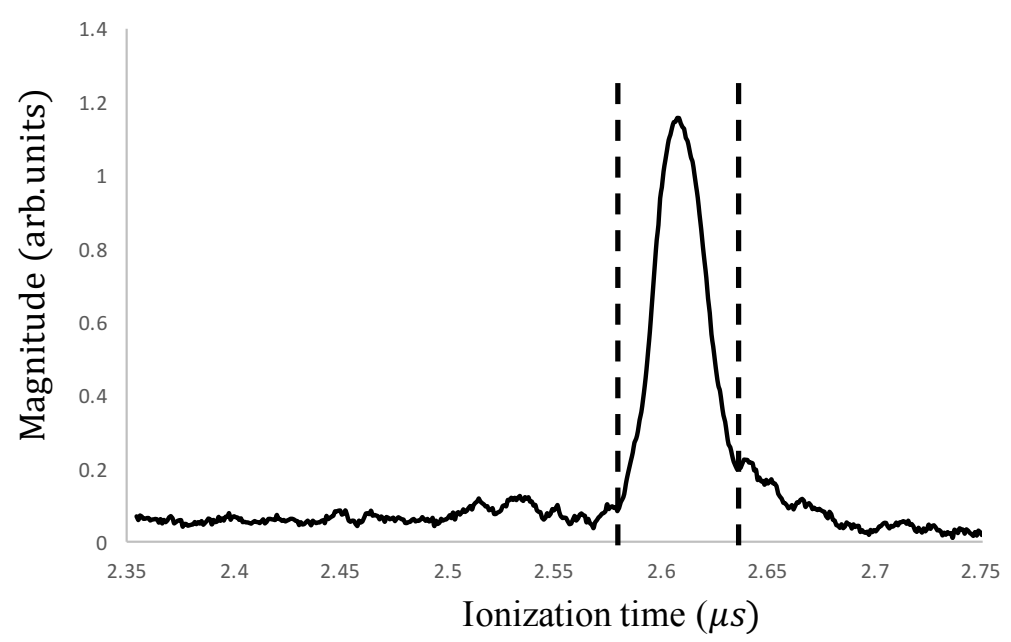

Figure 2.17: A typical ionization signal shown on an oscilloscope. The central peak representing the population of state $32 s$. The measurement program uses a user defined gate and integrates the area under the peak within the gate.

\subsection{Maintenance and Daily Operation}

Before doing experiments, participants must have finished the laboratory safety training.

Before turning on lasers, the laboratory main interlock switch has to be flipped on. It enables the lasers in the lab. When the switch is on, a red light bulb outside the lab is illuminated to indicate "laser on" status.

\subsubsection{Daily Examination}

1. Check the MOT chamber pressure. The reading from the ion pump should be no more than the order of $10^{-8}$ torr. A pressure reading higher than this indicates a problem, e.g. a vacuum leak or failing pump.

2. Check the pressure of the nitrogen tank which is used to keep the GCR-100 laser head clean and dry. The flow meter should read above zero and the output pressure on the 
tank should be around 5 psi. From previous experience, the nitrogen tank needs to be replaced every two to three weeks.

3. Check the room temperature. The readings should be from $72{ }^{0} \mathrm{~F}$ to $74{ }^{0} \mathrm{~F}$. On some days, the temperature may be out of this range, leading to laser and beam misalignment.

4. Check the temperature of the cooling water from external sources. The supply water should have a temperature around $60{ }^{0} \mathrm{~F}$.

5. Check the cooling water level within each laser or chiller before turning on the laser. The water level should be in the proper range marked on the reservoir.

6. Check the fume hoods to make sure they are working properly.

\subsubsection{Operation of the Regenerative Amplifier}

Turn on the seed light pump laser power switch. When the temperature is stabilized, turn on the laser. The pump should be in mode "power" and the setup for power is " 3.75 W" shown on the display screen.

1. Let the pump laser for the regenerative amplifier warm for at least half an hour. Then initiate the mode-locking. If the mode-lock is not very stable, usually it's because the laser alignment is off and it needs adjustment.

2. Turn on the regenerative pump GCR-100 Nd:YAG laser. Ensure beam blocks and/or the power meter head are in place, preventing light from entering either amplifier. Slowly increase the power of the pumping lamp until it reaches the maximum. It usually takes several seconds or minutes for the simmer light to turn on. If it takes too long, it's probably because there are too many ions in the cooling system and the charge of lamps is not working properly. Reflush the cooling system using deionized water if that happens and try again. 
3. Let the Nd:YAG laser warm for at least one hour to obtain thermal balance.

4. Change the output Pockels cell's timing to the long timing setpoint, which is 4 us longer than the short timing set point (which should be almost the same every day). This is to enable self lasing of the amplifier. Switch on all the Pockels cells in the setup. Increase the Nd:YAG pump light to be a little higher than the threshold. (The threshold may vary a little bit every day. The recent value should be marked down in the log book.)

5. There should be a bright spot showing in the TV monitor, which means the regenerative amplifier is now lasing itself. If there is no bright spot, increase the pump light a little higher but not larger than the normal pump level. Adjust the coupling mirrors to make sure the threshold is minimized.

6. Block the pump light. Change the output Pockels cell's timing to the short timing setting. Increase the pump light to the ordinary operation level (which is also written down in the log book). Unblock the pump light. Now on the scope, there should be a stably increasing pulse train, as observed on the photodiode detecting the light leakage through the regenerative amplifier cavity mirror.

At this point, the regenerative amplifier is ready. Fine tuning includes decreasing the threshold and making the pulse train more stable. The lamps in the Nd:YAG laser must be replaced every 700 hours, or so, under current repetition frequency. The normal output and the last replacement date are marked underneath the laser head on the optical table.

\subsubsection{Operation of the MOT}

The MOT is very sensitive to external perturbations. So during experiments, try not to make large noises or vibrations.

1. First turn on the cooling water valves. Check the flow meter to make sure cold water is flowing through the MOT coils' cooling tubes. If there is no flow or the flow is too 
slow, the power supply for the main anti-Helmholtz coils should not be turned on. Check the paddle wheel flow meter to make sure water is flowing. The normal input pressure is 14 psi. Make sure there is no leakage of water from the cooling tubes.

2. Turn on the diode lasers for the trap and repump beams.

3. Turn on the AOM driver, voltage ramp for the diode grating, scopes, TV monitors and coil power supplies. Increase the output of the power supply of the main coils to $10 \mathrm{~V}$. The resistance of the main coils is $1 \mathrm{ohm}$, so the output current of the power supply should be around 10A.

4. Turn on the $\mathrm{Rb}$ getter current and slowly increase it to the operating value. A normal operating current is from 1.9 A to $2.5 \mathrm{~A}$. When this value has to be as large as $3.5 \mathrm{~A}$ to generate an observable MOT on the TV monitor, it means the getter charge has been mostly used up. Under ordinary usage, this process could take about 4 to 5 years. Once the getter has been used up, it should be replaced by a new one.

5. Let the trap and repump lasers warm for at least one hour to achieve thermal balance. Then adjust the piezo voltage of the lasers to find the right absorption signal. If the absorption signal is not similar to the proper pattern, use a spectrometer to check the output frequency. For the trap laser, the output frequency range should cover the value 384232.6 GHz and for the repump laser $384231.2 \mathrm{GHz}$. If either diode laser fails to reach the required value, it is possible that the piezo in the laser head is damaged and needs to be replaced. Lock lasers.

At this point, there should be a bright spot shown on the TV monitors. It is the scattered infrared light from the cold atoms. A good MOT on the screen is a bright stable spot with a clear circular shape on both TV monitors. If the spot is not stable or the shape is not round, the first step is to adjust the current of the shim coils. If the shim coils do not do the job, 
usually it is because the alignment of the trap beams needs to be adjusted. Follow these troubleshooting procedures (refer to Figure 2.18):

1. Check the two windows for beams A and B respectively at the bottom of the chamber. There should be a mask on each window. These masks make the trap beam's spatial modes well defined.

2. There are four additional masks, two are big and two are small, which help to align the trap beams. Attach the two big masks to the front and the back windows, and adjust mirrors to make sure the trap beam $\mathrm{C}$ can go through the centers of the masks and be retro-reflected through them. Attach the two smaller ones to the top windows for beams A and B respectively. Make sure the beams' centers are the same as the masks' centers. Adjust mirrors so that beams A and B are retro-reflected. The retro-reflected beams should not totally overlap the incoming beams as feedback from the returning beams causes laser instability.

3. Take off the masks. Turn on the electronic switch (based on an IGBT transistor) which reduces the magnetic field to zero in $4 \mathrm{~ms}$ at a $15 \mathrm{~Hz}$ repetition rate. If the fluoresce signal is not a round spot but rather has an irregular shape with one or more "tails", indicating the directions the atoms in the MOT tend to escape, then adjust the shim coils and the mirrors to get rid of these "tails".

4. Turn off the magnetic field switch. Now the MOT signal monitored by the camera should be a bright spot. If it is still not very steady or has blurry edges, fine tune the current to the shim coils to improve it.

5. Adjust the repump beam to find the best performance of the MOT. The repump alignment does not affect the MOT much, so this step is not often required. 


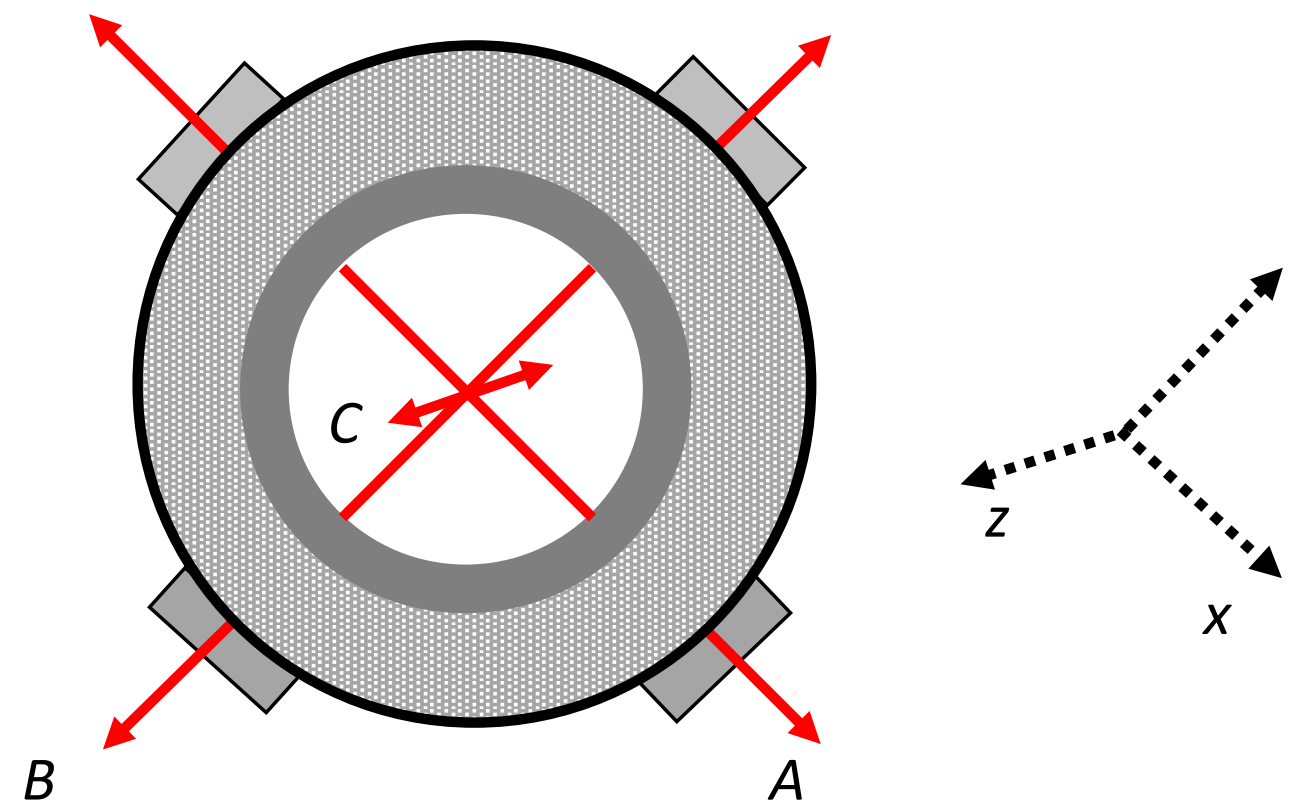

Figure 2.18: Front view of the chamber that houses the MOT. A, B, and C represent the three trap beams. 


\section{Bibliography}

[1] E. L. Raab, M. Prentiss, Alex Cable, Steven Chu, and D. E. Pritchard. Trapping of Neutral Sodium Atoms with Radiation Pressure. Phys. Rev. Lett. 59, 2631 (1987).

[2] Harold J. Metchalf and Peter van der Straten. Laser Cooling and Trapping. Springer (1999).

[3] W. Anderson, PhD thesis, University of Virginia (1996).

[4] Mary Kutteruf, Coherence in Rydberg Atoms: Measurement and Control, $\mathrm{PhD}$ thesis, University of Virginia (2010).

[5] Continuum, Mountain View, CA. Operation and Maintenance Manual for SureLite Lasers (1997).

[6] Spectra-Physics, Mountain View, CA. Pulsed ND:YAG Lasers User's Manual GCR100 Series GCR-200 Series (1993).

[7] T.W. Hansch. Repetitively Pulsed Tunable Dye Laser for High Resolution Spectroscopy. Applied Optics, 11:895 (1972).

[8] János Hebling, Ka-Lo Yeh, Matthias C. Hoffmann, Balázs Bartal, and Keith A. Nelson, Generation of High-Power Terahertz Pulses by Tilted-Pulse-Front Excitation and Their Application Possibilities, J. Opt. Soc. Am. B, 25, B6 (2008).

[9] Sha Li, THz Induced Field Ionization and Field Emission, PhD thesis, University of Virginia (2016).

[10] Mukesh Jewariya, Masaya Nagai, and Koichiro Tanaka. Enhancement of Tera-Hertz Wave Generation by Cascaded $\chi^{2}$ Processes in $\mathrm{LiNbO}_{3}$. J. Opt. Soc. Am. B, 26, 9 (2009). 
[11] János Hebling, Gábor Almási, Ida Z. Kozma, and Jürgen Kuhl. Velocity Matching by Pulse Front Tilting for Large-Area Thz-pulse Generation. Opt. Express, 10, 21 (2002).

[12] Thomas F. Gallagher. Rydberg Atoms. Cambridge University Press (1994).

[13] Kapteyn-Murnane Laboratory Inc, Model MTS Mini Ti:Sapphire Laser Kit Instruction Manual (2003).

[14] Q. Wu, X. - C. Zhang, Free-space Electro-Optic Sampling of Terahertz Beams, Appl. Phys. Lett. 67, 3523 (1995). 


\section{Models in Simulation}

This chapter introduces general knowledge about the computational models used in the research. The topics include Rydberg atoms, a two-atom model, a dipole-dipole interaction model, blackbody radiation, etc. The mathematical equations presented provide a background for the simulations implemented in chapters 4,5 and 6 . Unless specifically noted, atomic units are used throughout this dissertation. 


\subsection{Rydberg Atoms}

Back in 1885, Balmer found the wavelengths of the visible series of atomic $\mathrm{H}$ are given by [1]:

$$
\lambda=\frac{b n^{2}}{n^{2}-4}
$$

where $b=3464.6 \AA$. We now know equation (3.1) is the formula for the wavelengths of the Balmer series of transitions between the $\mathrm{n}=2$ states and higher lying levels.

After quantitatively describing the wavelengths from $\mathrm{H}$, people started to work on other atoms to unravel the mystery of atomic spectroscopy. Living and Dewar found that the observed spectral lines of $\mathrm{Na}$ could be grouped into different series [2].Hartley found the significance of describing Balmer's formula in terms of the wavenumber or frequency of the observed lines instead of the wavelength during his reach on spectra of $\mathrm{Mg}, \mathrm{Zn}$, and $\mathrm{Cd}$ [3]:

$$
v=\frac{1}{4 b}\left(\frac{1}{4}-\frac{1}{n^{2}}\right) .
$$

This equation makes it more clear that the lines Balmer discovered reflect the energy differences between the $n=2$ and higher lying levels.

Following those pioneering measurements, Rydberg began to classify the spectra of other atoms, notably alkali atoms, into sharp, principal, and diffuse series of lines [4]. For example, he found the frequencies associated with transitions between the $s$ and $p$ series, are given by:

$$
\pm v=\frac{\mathrm{Ry}}{\left(\mathrm{m}-\delta_{\mathrm{s}}\right)^{2}}-\frac{\mathrm{Ry}}{\left(\mathrm{n}-\delta_{\mathrm{p}}\right)^{2}}
$$

where the + sign and constant $n$ describe sharp transitions involving series of $s$ states, and the minus sign and a constant $m$ describe transition involving the principal series of $p$ states. If $\delta_{s}=\delta_{p}=0$ and $m=2$ we recover Balmer's formula for the $\mathrm{H}$ transition from $n=2$. 
Due to his significant contribution, people now refer to atoms in states of high principal quantum number as "Rydberg Atoms".

\subsubsection{Modern Picture of Rydberg Atoms}

If we consider Rydberg states of $\mathrm{H}$ and $\mathrm{Na}$, as shown in Figure 3.1, they are quite similar. The only difference is that $\mathrm{Na}$ atom has a core which is composed of 11 positive charges and 10 electrons. In classical terms, at most of times, the high energy Rydberg electron is far from the core, where the difference between $\mathrm{Na}, \mathrm{H}$ and all Rydberg atoms is trivial. But when the Rydberg electron comes near the core, it can both polarize and penetrate the $\mathrm{Na}^{+}$core, altering the wavefunctions and energies of $\mathrm{Na}$ Rydberg states relative to their hydrongenic counterparts.
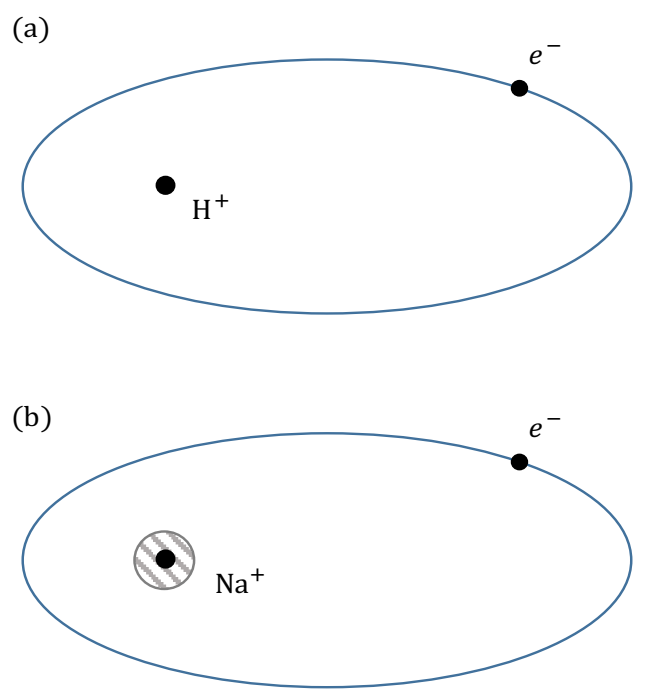

Figure 3.1: Classical view of Rydberg orbits of (a) $\mathrm{H}$ and (b) Na. In $\mathrm{H}$ the electron orbits around the proton. In $\mathrm{Na}$ it orbits around the +11 nuclear charge and ten inner shell electrons. In high $\ell$ states, $\mathrm{Na}$ behaves nearly identically to $\mathrm{H}$, but in low $\ell$ states the $\mathrm{Na}$ electron penetrates and polarizes the inner shell electrons of the $\mathrm{Na}^{+}$core [5].

We know how to calculate wavefunctions of $\mathrm{H}$ [6]. This process can be easily extended to generate wavefunctions for single valence electron atoms with spherical ionic cores. Such an approach is called Quantum Defect Theory [7]. Quantum Defect Theory (QDT) 
assumes that the $\mathrm{Na}^{+}$core is spherically symmetric and frozen in place. So the effective potential, $V_{N a}$ seen by the valence electron is spherically symmetric and only depends on $r$. This potential is different from the coulomb $-1 / r$ potential only at small $r$. Typically, the effect is to increase the electron's kinetic energy and decrease the wavelength of the radial oscillations in the wavefunction relative to $\mathrm{H}$. In general, the bound state radial wavefunctions are given by:

$$
\rho(r)=f(W, \ell, r) \cos \tau-g(W, \ell, r) \sin \tau
$$

where $f(W, \ell, r)$ and $g(W, \ell, r)$ are commonly termed the regular and irregular coulomb functions and $\tau$ is the phase shift which is related to the quantum defect, $\delta_{\ell}$. For a give principal quantum number, the Rydberg atom has allowed eigen energies:

$$
W_{n}=-\frac{1}{2\left(n-\delta_{\ell}\right)^{2}}
$$

where $n$ is an integer. Equation (3.5) can be used to calculate the energies of Rydberg atoms if the quantum defects $\delta_{\ell}$ are known. Table 3.1 gives the $0^{\text {th }}$ order approximation of the quantum defects for $\mathrm{Rb}$.

\begin{tabular}{c|c|c|c|c|c}
\hline & $s_{1 / 2}$ & $p_{1 / 2}$ & $p_{3 / 2}$ & $d_{3 / 2,5 / 2}$ & $f_{5 / 2,7 / 2}$ \\
\hline$\delta_{\ell}$ & 3.13109 & 2.65456 & 2.64145 & 1.347157 & 0.016312 \\
\hline
\end{tabular}

Table 3.1:Quantum defects for low- $\ell$ states of $\mathrm{Rb}$ [5].

From Table 3.1 we see that, for larger $\ell$, the quantum defect is smaller. This is to be expected as the centrifugal barrier reduces the probability of finding the electron near the nucleus as $\ell$ increases. 


\subsubsection{Properties of Rydberg Atoms}

Table 3.2 summarizes the dependence on principal quantum number $n$ of some properties of Rydberg atoms [12].

\begin{tabular}{l|c}
\hline property & $n$ dependences \\
\hline Binding energy & $n^{-2}$ \\
Energy between adjacent $n$ states & $n^{-3}$ \\
Orbital radius & $n^{2}$ \\
Orbital period & $n^{3}$ \\
Geometric cross section & $n^{4}$ \\
Dipole moment $<n s|\mathrm{e} r| n p>$ & $n^{2}$ \\
Polarizability & $n^{7}$ \\
Radiative lifetime & $n^{3}$ \\
Fine-structure interval & $n^{-3}$ \\
\hline
\end{tabular}

Table 3.2: Properties of Rydberg Atoms.

Because Rydberg wavefunctions extend far from the ion core, they are easily affected by external fields, including the fields created by other atoms. For example, as introduced in later sections, the magnitude of the dipole-dipole interaction between Rydberg atoms is proportional to the product of two transition dipole moments and, therefore, scales as $n^{4}$ for atoms in states of similar $n$. In addition, the very long lifetime of Rydberg atoms makes it possible to perform complex control operations on the the atoms prior to their decay. These superior properties make them ideal objects for researching coherent dipole-dipole interactions and the control of electron dynamics. 


\subsection{Two-Atom Model}

\subsubsection{Introduction to the Two-Atom Model}

When talking about the interactions (which will be introduced in detail in 3.3) between atoms, a simplified two-atom DD model is often used. In this model, we suppose one atom is only affected by its nearest neighbor. Such an assumption is not completely accurate of course, because nearest neighbor interactions do not preclude the influence from other atoms. However, due to the inverse cube dependence of the DD coupling on atom separation, nearest neighbor interactions are typically several times larger than the nextnearest neighbor couplings. Moreover, compared to a many-atom picture, the two-atom model provides a concise way of thinking about and charactering interactions between atoms in an ensemble [5]. In addition, at sufficiently low densities where the atom separation is much larger than extent of the Rydberg wavefunctions, the principal atomatom interaction is of the DD form [9]. As discussed later, in the experiments described here, the interaction between nearest neighbor atoms dominates over interactions with more distant neighbors. Therefore, the following discussion focuses on the coupling between nearest neighbor pairs. 


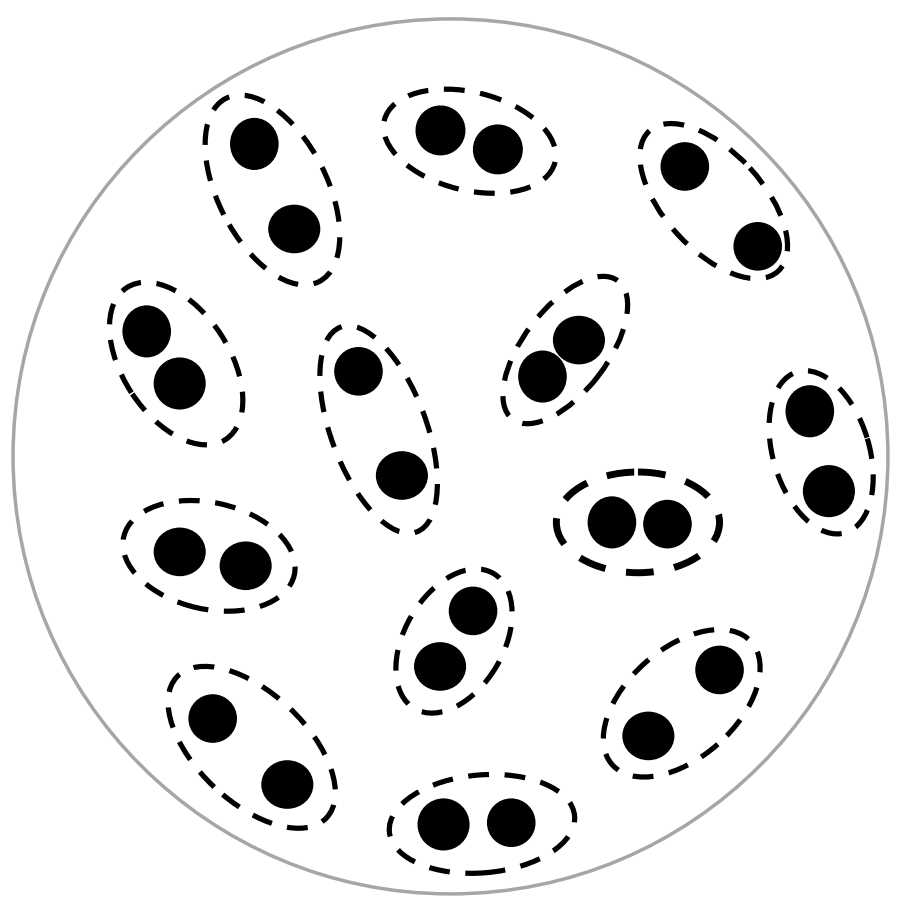

Figure 3.2: Schematic of atom pairs in a MOT. For each atom in the MOT, we only consider the effect of its nearest neighbor. One atom and its nearest neighbor is considered to be "a pair of atoms".

For each pair of atoms, we can write their electronic state, in a non-interacting basis, as the product of their individual states. For example, for two atoms, one in an $n s$ state and the other in an $n p$ state, respectively, we can write the state of the pair as $n s n p$. We call this state a "pair state". Such a convention is followed in all sections of this dissertation.

\subsubsection{Nearest Neighbor Distribution}

We need to know the distance between atoms to determine the DD coupling between them. We use the so called "nearest neighbor distribution" to express the probability that an atom and its nearest neighbor in a random ensemble have a particular separation. The nearest neighbor distribution function $H(r)$ for point particles in a D-dimensional system is [12][13]: 


$$
H(r)=\rho \frac{\mathrm{d} v_{D}(r)}{\mathrm{d} r} \exp \left[-\rho v_{D}(r)\right]
$$

where $\rho$ is the density of the atoms and $v_{D}(r)$ is the volume of the D-dimensional sphere. For a 3-D system, we get the distribution function:

$$
H(r)=4 \pi \rho r^{2} \exp \left[-\frac{4}{3} \pi \rho r^{3}\right]
$$

From Equation (3.7), we find that the most possible nearest neighbor distance between atoms in a random ensemble is:

$$
R \simeq \sqrt[3]{\frac{1}{2 \pi \rho}}
$$

For a MOT with density $\rho \approx 10^{9} \mathrm{~cm}^{-3}$ and a temperature $\mathrm{T} \sim 100 \mu \mathrm{K}$, the most possible distance between one $\mathrm{Rb}$ atom and its nearest neighbor is about $5 \mu \mathrm{m}$ and the velocity of the atoms is on the order of $10 \mathrm{~cm} / \mathrm{s}$. In $1 \mu \mathrm{s}$, each atom can move about $0.1 \mu \mathrm{m}$ on average, which is much smaller than the distance between two atoms. So we consider the atoms as "frozen" or static in the MOT for experiments involving DD integration times $<1 \mu \mathrm{s}$.

\subsubsection{Förster Resonant Energy Transfer}

Förster Resonant Energy Transfer (FRET) is a mechanism describing energy transfer between two atoms or molecules. It happens when two neighboring atoms are dipole-dipole coupled to higher and lower states with equal energy spacing [10][14][20]. This mechanism can be described very easily using the two-atom picture. As shown in Figure 3.3, one atom acts as an energy donor and the other one, an accepter. The atoms exchange energy as the donor is de-excited to a lower state and the accepter is excited to a higher state via the DD interaction. 
(a)

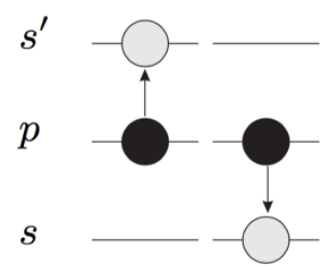

(b)

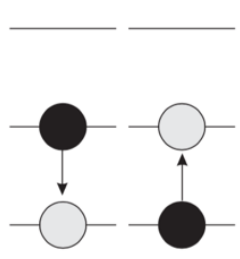

(c)

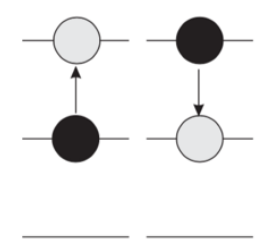

Figure 3.3: Schematic for typical FRET. Black circles represent the initial pair states and gray circles the final pair states. (a) is $p p \rightarrow s s^{\prime}$, (b) $p s \rightarrow s p$ and (c) $p s^{\prime} \rightarrow s^{\prime} p$ [14].

\subsection{Dipole-Dipole Interaction}

In the above discussion, the DD interaction has been mentioned several times. For Rydberg atoms in the two-atom approximation, it is straightforward to explicitly derive the form of the interaction.

\subsubsection{Dipole Moment}

As noted previously, a classical Rydberg electron spends most of the time far from the positive core that binds it. Although the atom as a whole is neutral, classically it has a time dependent dipole moment:

$$
\vec{\mu}=q \vec{r}
$$

where $q$ is the electron charge and $\vec{r}$ is the vector from the negative charge to the positive charge. This classical physics perspective can help us understand the dipole-dipole interaction better.

In quantum physics, in the absence of external fields, an atom in an electronic eigenstate has no permanent dipole moment. However, an external electric field, or neighboring atoms can induce a dipole moment. A state which is a linear combination of angular moment 
eigenstates of different parity, can exhibit a permanent or time dependent dipole moment. This dipole moment depends on the "transition dipole moment" between different field free states:

$$
\overrightarrow{\mu_{12}}=\langle 1|\vec{r}| 2\rangle
$$

for $q=e=1$. From Equation (3.10) we see that if state 1 and state 2 are the same angular momentum eigenstates, then the transition dipole moment is 0 . Thus the transition dipole moment appears as an off-diagonal matrix element in the system Hamiltonian.

The preceding discussion on atomic dipole moments has been divided into classical and quantum physics views. Similar to that, our DD interaction model will also be explored from both views.

\subsubsection{Dipole-Dipole Interaction in the Classical Picture}

The classic dipole-dipole interaction picture provides the basis for the interaction between quantum atoms, and helps us to understand the quantum picture.

If there is only one dipole, the potential of this dipole is merely provided by the attractive force between the positive charge and negative charge. When two dipoles are in close proximity, the potential energy of such a system also induces interactions between them. Figure 3.4 illustrates the interaction between two dipoles. The total potential energy of this system does not only contain the inner coulomb potential of each individual dipole but also the potential caused by atom-atom interaction. The atom-atom interaction potential energy can be written as:

$$
V=\frac{1}{R}-\frac{1}{\left|\overrightarrow{r_{1}}-\vec{R}\right|}-\frac{1}{\left|\overrightarrow{r_{2}}+\vec{R}\right|}+\frac{1}{\left|\overrightarrow{r_{1}}-\overrightarrow{r_{2}}-\vec{R}\right|}
$$

From reference [15], assuming $r_{1}, r_{2} \ll R$, Equation (3.11) can be simplified to: 


$$
V \approx V_{D D}=\frac{\overrightarrow{r_{1}} \cdot \overrightarrow{r_{2}}-3\left(\overrightarrow{r_{1}} \cdot \hat{R}\right)\left(\overrightarrow{r_{2}} \cdot \hat{R}\right)}{R^{3}}
$$

where $V_{D D}$ is the dipole-dipole interaction.

Expressed in terms of dipole moments, Equation (3.12) can also be written as:

$$
V_{D D}=\frac{\overrightarrow{\mu_{1}} \cdot \overrightarrow{\mu_{2}}-3\left(\overrightarrow{\mu_{1}} \cdot \hat{R}\right)\left(\overrightarrow{\mu_{2}} \cdot \hat{R}\right)}{R^{3}}
$$

where $\overrightarrow{\mu_{1}}$ is the dipole moment of atom 1 and $\overrightarrow{\mu_{2}}$ is the dipole moment of atom 2 . From this expression, we see that the interaction energy can be positive or negative depending on the relative orientation of the dipole moments and the line connecting them.

From Equation (3.13) we can also see the dipole-dipole interaction is strongly affected by the distance between the dipoles or Rydberg atoms. This is one reason why the nearest neighbor interaction typically dominates in an ensemble of frozen atoms.

Setting $\hat{R}=\hat{z}$, Equation (3.12) can be written as:

$$
V_{D D}=\frac{x_{1} x_{2}+y_{1} y_{2}-2 z_{1} z_{2}}{R^{3}}
$$

where $x_{i}, y_{i}$, and $z_{i}$ specify the position of the Rydberg electron in the ith atom relative to its nucleus.

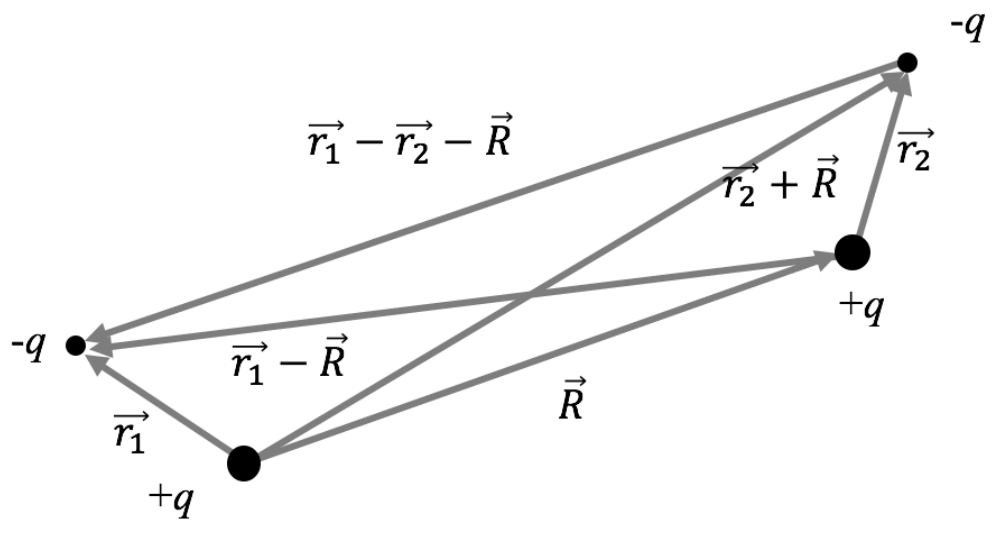

Figure 3.4: Schematic of the interaction between two classical dipoles. 


\subsubsection{Dipole-Dipole Interaction in the Quantum Picture}

The quantum DD model derives from the classical picture. As an example, we consider a resonant interaction such as that shown in Figure 3.3a. Suppose an atom pair is initially in state $p p$ (ignoring $m_{\ell}$ ), and we want to explore the evolution of the wavefunction in such a system. We define:

$$
\psi_{A}=p p, \text { and } \psi_{B}=s s^{\prime} .
$$

The total Hamiltonian of the system is $H=H_{0}+V$, where $V$ is the potential associated with the classical dipole-dipole interaction. The solution of the time-dependent Schrodinger equation, $H \Psi=i \partial \Psi / \partial t$ yields the pair wavefunction which, in general, is a linear combination of $\psi_{A}$ and $\psi_{B}$ :

$$
\Psi=C_{A}(t) \psi_{A}+C_{B}(t) \psi_{B}
$$

where all of the time dependence is contained in the coefficients $C_{A}(t)$ and $C_{B}(t)$. The Schrodinger equation can be written as:

$$
\left(\begin{array}{cc}
E_{A}+V_{A A} & V_{A B} \\
V_{B A} & E_{B}+V_{B B}
\end{array}\right)\left(\begin{array}{c}
C_{A} \\
C_{B}
\end{array}\right)=i\left(\begin{array}{c}
\dot{C}_{A} \\
\dot{C}_{B}
\end{array}\right)
$$

where $V_{p q}=\left\langle\psi_{p}|V| \psi_{q}\right\rangle$, with $p$ and $q$ representing pair states. $V_{A A}=V_{B B}=0$. By solving Equation (3.17) we can get two eigen energy values:

where $\varepsilon$ is expressed as:

$$
\left\{\begin{array}{l}
E_{1}=\frac{\left.\left(E_{A}+E_{B}\right)+\sqrt{\left(E_{A}+E_{B}\right)^{2}-4\left(E_{A} E_{B}-\varepsilon^{2}\right.}\right)}{2} \\
E_{2}=\frac{\left.\left(E_{A}+E_{B}\right)-\sqrt{\left(E_{A}+E_{B}\right)^{2}-4\left(E_{A} E_{B}-\varepsilon^{2}\right.}\right)}{2}
\end{array}\right.
$$

$$
\varepsilon=\left\langle p p|V| s s^{\prime}\right\rangle \propto \frac{\left\langle p\left|\overrightarrow{r_{1}}\right| s\right\rangle \cdot\left\langle p\left|\overrightarrow{r_{2}}\right| s^{\prime}\right\rangle}{R^{3}}=\frac{\overrightarrow{\mu_{p s}} \cdot \overrightarrow{\mu_{p s^{\prime}}}}{R^{3}} .
$$


This expression utilizes the concept of transition dipole moment mentioned in section 3.3.1.

When in resonance, $E_{A}=E_{B}=E$, Equation (3.18) evolves to:

$$
\left\{\begin{array}{l}
E_{1}=E+\varepsilon \\
E_{2}=E-\varepsilon
\end{array}\right.
$$

We can also obtain the two eigenstates from Equation (3.17) at resonance:

$$
\left\{\begin{array}{l}
\psi_{1}=\left(\psi_{A}+\psi_{B}\right) / \sqrt{2} \\
\psi_{2}=\left(\psi_{A}-\psi_{B}\right) / \sqrt{2}
\end{array} .\right.
$$

So the complete solution of the Equation (3.17) is:

$$
\Psi=C_{1} \psi_{1} e^{-i E_{1} t}+C_{2} \psi_{2} e^{-i E_{2} t}
$$

in which $C_{1}$ and $C_{2}$ are constants determined by the initial condition.

The energy shifts that occur in the presence of resonant interactions act to decouple nearest neighbor pairs from surrounding atoms. As a result, the dynamics are dominated by the nearest neighbor coupling [9][11].

The above discussion gives the approach for dealing with DD interactions in a simple two-state system. To calculate the matrix elements for such a system and more complicated systems, we need to go deeper and consider the relative orientation of the atomic dipole relative to the inter nuclear axis. By separating the radial part and the angular parts of the interaction matrix element, the calculation is implemented as follows.

From Equation (3.14), using the relations between the Cartesian coordinates and the spherical harmonics $\left(Y_{l m}\right)$,

$$
x=\sqrt{\frac{2 \pi}{3}} r\left(-Y_{11}+Y_{1-1}\right)
$$




$$
\begin{aligned}
& y=i \sqrt{\frac{2 \pi}{3}} r\left(Y_{11}+Y_{1-1}\right) \\
& z=\sqrt{\frac{4 \pi}{3}} r Y_{10}
\end{aligned}
$$

the DD interaction can be written in terms of spherical harmonics as:

$$
V_{D D}=-\frac{r_{1} r_{2}}{R^{3}} \cdot \frac{4 \pi}{3} \cdot\left({ }^{1} Y_{11}{ }^{2} Y_{1-1}+{ }^{1} Y_{1-1}{ }^{2} Y_{11}+2{ }^{1} Y_{10}{ }^{2} Y_{10}\right)
$$

where ${ }^{i} Y_{l m}$ is a spherical harmonic operator acting on the Rydberg electron of the $i$ th atom, and $r_{j}$ is the purely radial part of the transition matrix element in atom $j$.

To compute the matrix elements, Edmond's $C$-tensors are introduced [16]. The relation between the spherical harmonic tensors and the $C$-tensors is:

$$
C_{q}^{k}=\sqrt{\frac{4 \pi}{2 k+1}} Y_{k q}
$$

So the dipole-dipole interaction in form of $C$-tensors is:

$$
V_{D D}=-\frac{r_{1} r_{2}}{R^{3}}\left(C_{1}^{1} C_{-1}^{1}+C_{-1}^{1} C_{1}^{1}+2 C_{0}^{1} C_{0}^{1}\right)
$$

In Equation (3.26), a pair of $C$ 's implies that the first $C$ acts on the first atom and the second $C$ on the second atom.

Equation (3.26) is used in the simulations described in forthcoming chapters to calculate the DD matrix element. As an example, the DD matrix element between $p_{\frac{11}{2} 2} p_{\frac{1}{2} \frac{1}{2}}$ and $s_{\frac{1}{2}} \frac{1}{2} S_{\frac{1}{2}}^{\prime} \frac{1}{2}$ is expressed as:

$$
\left\langle p_{\frac{1}{2} \frac{1}{2}} p_{\frac{1}{2} \frac{1}{2}}\left|V_{D D}\right| s_{\frac{1}{2} \frac{1}{2} \frac{1}{2} s_{\frac{1}{2}}^{\prime}}^{\prime}\right\rangle=-\frac{r_{1} r_{2}}{R^{3}}\left\langle p_{\frac{11}{22}}\left|C_{0}^{1}\right| s_{\frac{1}{2} \frac{1}{2}}\right\rangle\left\langle p_{\frac{11}{22}}\left|C_{0}^{1}\right| s_{\frac{1}{2} \frac{1}{2}}^{\prime}\right\rangle
$$

where the state of each atom is expressed as $\ell_{j m_{j}}$. The radial part of the matrix elements are calculated using a numerical Numerov integration algorithm [19]. 


\subsubsection{Dipole-Dipole Coupled System in an Electric Field}

In some of the experiments, we utilize $\mathrm{THz}$ pulses to coherently redistribute population among Rydberg states. The THz pulse can be treated as a time-dependent electric field. To simulate the redistribution process, we need to model the effect of the $\mathrm{THz}$ field in the presence of the DD coupling.

The model is based on the picture shown in Figure 3.5. We define the vector pointing from one atom core to the other core as the $z$-axis. The electric field has an angle $\theta$ relative to the $z$ axis. We define the plane containing the electric field vector and the $z$ axis as the $x z$ plane.

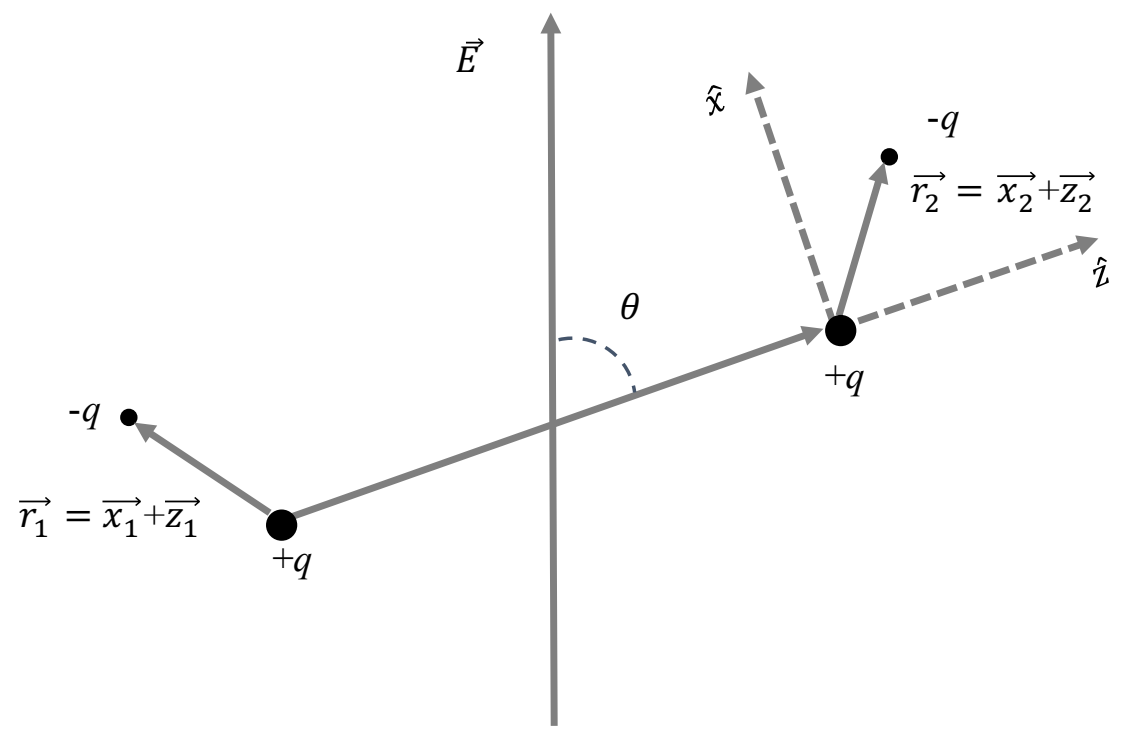

Figure 3.5: Schematic for a DD system in an electric field.

As shown in Figure 3.5, for the electron in atom 1, the interaction with the electric field has the form:

$$
H_{1}=-q \vec{E} \cdot \overrightarrow{r_{1}}=-q E_{0}\left(\sin \theta \overrightarrow{x_{1}}+\cos \theta \overrightarrow{z_{1}}\right)
$$

where $\mathrm{E}_{0}$ is the amplitude of the $\mathrm{THz}$ field.

From Equation (3.23) and (3.25), the above equation can be re-expressed as: 


$$
H_{1}=-q \vec{E} \cdot \overrightarrow{r_{1}}=-q E_{0} r_{1}\left(\frac{\sin \theta}{\sqrt{2}}\left(C_{1}^{1}+C_{-1}^{1}\right)+\cos \theta C_{0}^{1}\right)
$$

For a randomly distributed ensemble composed of many pairs of atoms, each pair has its own defined $z$ axis and it is chosen as the quantization axis for that pair, thus $\theta$ is different for different pairs.

A similar expression applies to atom 2. So the Hamiltonian associated with the applied electric field in a DD system is:

$$
H_{E}=H_{1}+H_{2}
$$

We already know the form of DD interaction. Using perturbation theory or numerical integration, we can calculate the evolution of wavepackets in a random ensemble under the influence of dipole-dipole interaction, as well as the state redistribution when the $\mathrm{THz}$ pulses are applied to the atoms.

\subsection{Rydberg Decay Model}

Rydberg atoms are strongly affected by blackbody radiation, even at room temperature. The strong influence of thermal radiation is due to two reasons [5]. First, the energy spacing between adjacent Rydberg levels is small $\left(\Delta E \propto n^{-3}\right)$, so that $\Delta E$ can be $<\mathrm{KT}$ at $300 \mathrm{~K}$. Second, the dipole matrix elements for transitions between adjacent Rydberg states are enormous, scaling as $n^{2}$, providing a large coupling between atoms and the thermal radiation. Because of this strong coupling, population initially in one state can rapidly diffuse to other energetically nearby states due to blackbody radiation. 


\subsubsection{Blackbody Induced Transitions}

The spontaneous decay rate of a state $n \ell$ to a lower lying state $n^{\prime} \ell^{\prime}$ is given by the Einstein $A$ coefficient $A_{n^{\prime} \ell^{\prime}, n \ell}$ [17]. The thermal radiation induced stimulated emission rate is:

$$
K_{n^{\prime} \ell^{\prime}, n \ell}=\bar{n} A_{n^{\prime} \ell^{\prime}, n \ell}
$$

where $\bar{n}$ is the photon occupation number [18] of the thermal radiation,

In terms of the average oscillator strength, Equation (3.31) can be reexpressed as:

$$
K_{n^{\prime} \ell^{\prime}, n \ell}=2 \bar{n} \alpha^{3} w_{n^{\prime} \ell^{\prime}, n \ell}^{2}\left|\bar{f}_{n^{\prime} \ell^{\prime}, n \ell}\right|
$$

where $\alpha$ is the fine structure constant, $\mathrm{w}_{n^{\prime} \ell^{\prime}, n \ell}$ is the energy difference $W_{n^{\prime} \ell^{\prime}}-W_{n \ell}$,

$$
\bar{f}_{n^{\prime} \ell^{\prime}, n \ell}=\frac{2}{3} w_{n^{\prime} \ell^{\prime}, n \ell} \ell_{\max }^{2 \ell+1}\left|\left\langle n^{\prime} \ell^{\prime}|r| n \ell\right\rangle\right|^{2},
$$

and $\ell_{\max }$ is the larger of $\ell$ and $\ell^{\prime}$ [5].

Equation (3.32) and Equation (3.33) are used in Chapter 4 to calculate the blackbody radiation induced transition rate between nearby Rydberg levels.

\subsubsection{Radiative Transitions}

When considering the decay of the population from a single Rydberg state, as described in Chapter 4, radiation models similar to the schematic in Figure 3.6 are used. There is spontaneous decay into lower lying states. There are blackbody induced transitions between the starting state and its higher and lower energy neighbors. There is also blackbody radiation between one neighbor and the neighbor's neighbors, and so on. 


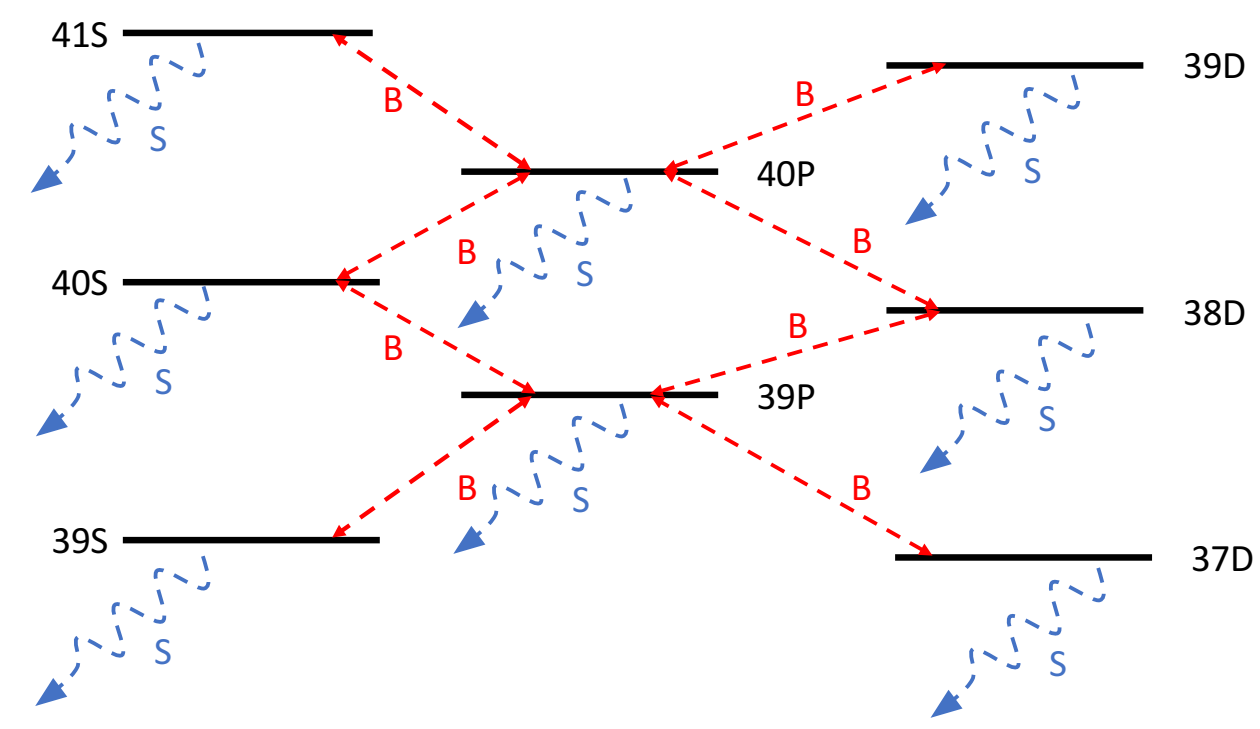

Figure 3.6: Decay model for atoms starting from the $40 s$ state as an example. The red dash lines between two states indicate blackbody stimulated transitions between those two states. The blue dashed curves represent spontaneous decay.

In reality, there are more states involved in the decay and transition process and the situation is much more complicated. But for calculation convenience, a limited number of nearby states are chosen. To make sure such a simplification is reasonable, results based on different numbers of states are compared to find an essential set of nearby states for which the calculations agree well with those performed with additional states. 


\section{Bibliography}

[1] H. E. White, Introduction to Atomic Spectra, McGraw-Hill, New York (1934).

[2] G. D. Living and J. Dewa, Proc, Roy, Soc. Lond, 29, 398 (1879).

[3] W. N. Hartley, J. Chem. Soc. 43, 390 (1883).

[4] J. R. Rydberg, Phil. Mag. $5^{\text {th }}$ Ser. 29, 331 (1890).

[5] Thomas F. Gallagher. Rydberg Atoms. Cambridge University Press (1994).

[6] R. Shankar, Principles of Quantum Mechanics, $2^{\text {nd }}$ Edition, Plenum Press (1994).

[7] M. J. Seaton, Rep. Prog. Phys. 46, 167 (1983).

[8] Thomas F. Gallagher. Rep. Prog. Phys. 51, 143 (1988).

[9] B. Sun and F. Robicheaux, Spectral Linewidth Broadening from Pair Fluctuations in A Frozen Rydberg Gas, Phys. Rev. A. 78, 040701 (2008).

[10] I. Mourachko, D. Comparat, F. de Tomasi, A. Fioretti, P. Nosbaum, V. M. Akulin, and P. Pillet, Many-Body Effects in a Frozen Rydberg Gas, Phys. Rev. Lett. 80, 253 (1998).

[11] K. C. Younge, A. Reinhard, T. Pohl, P. R. Berman, and G. Raithel, Mesoscopic Rydberg Ensembles: Beyond the Pairwise-interaction Approximation, Phys. Rev. A. 79,043420 (2009).

[12] S Torquato, B Lu and J Rubinstein, Nearest-neighbour Distribution Function for Systems of Interacting Particles, J. Phys. A: Math. Gen. 23 (1990)

[13] Hertz P, Math. Ann. 67, 387 (1909).

[14] S. Westermann, T. Amthor, A.L. de Oliveira, J. Deiglmayr, M. Reetz-Lamour, and M. Weidemuller, Dynamics of resonant energy transfer in a cold Rydberg gas, Eur. Phys. J. D 40, 37-43 (2006). 
[15] F. Robicheaux, J. V. Hernández, T. Topçu,1 and L. D. Noordam, Simulation of Coherent Interactions between Rydberg Atoms, Phys. Rev. A. 70, 042703 (2004).

[16] A. R. Edmonds, Angular Momentum in Quantum Mechanics, Princeton University Press, Princeton (1960).

[17] H. A. Bethe and E. A. Salpeter, Quantum Mechanics of One-and-Two-Electron Atoms, Academic Press, New York (1957).

[18] R. Loudon, The quantum Theory of Light, Oxford University Press, London (1973).

[19] M. L. Zimmerman, M. G. Littman, M. M. Kash, and D. Kleppner, Rydberg States of Atoms and Molecules, Phys. Rev. A 20, 2251 (1979).

[20] W.R. Anderson, J.R. Veale, and T.F. Gallagher, Phys. Rev. Lett. 80, 249 (1998). 


\title{
4 Absence of Collective Decay in a Cold Rydberg Gas
}

\begin{abstract}
We have studied the decay of Rydberg excitations in a cold Rb gas. A $10 \mathrm{~ns}$, pulseddye-amplified diode laser excites $\mathrm{Rb}$ atoms at $70 \mu \mathrm{K}$ in a magneto-optical trap to $n s$ or $n p$ Rydberg states with principal quantum numbers $26 \leq n \leq 40$. Time-delayed stateselective field ionization is used to directly monitor the population in the initial and neighboring Rydberg levels. The measured time dependence of the Rydberg population is well described by numerical simulations which consider only spontaneous emission and population transfer by blackbody radiation. No evidence for collective decay is found at atom densities up to $3 \times 10^{9} \mathrm{~cm}^{-3}$. This result is in contrast to a previous study [Wang et al., Phys. Rev. A 75, 033802 (2007)], in which superradiant decay was theoretically predicted and experimentally inferred for atom density and laser focal volume conditions very similar to those considered here. Suppression of collective emission is likely due to variations in transition energies within the atom sample, dominated by inhomogeneities in dipole-dipole exchange interactions for initial $s$ states, or by a combination of dipole-dipole and electric field inhomogeneities for the initial $p$ states.
\end{abstract}




\subsection{Introduction}

Atoms within cold Rydberg ensembles are coupled by strong long-range dipole-dipole (DD) interactions [1], making them interesting systems for exploring few- and many-body quantum dynamics in general and applications in quantum information in particular [2-35] (The properties of Rydberg Atoms are described in Chapter 3). Of course, unlike in groundstate systems, finite Rydberg lifetimes limit the types of measurements and number of coherent manipulations that can be performed in a given experiment. Fortunately, isolated Rydberg atoms exhibit low spontaneous decay rates [1], potentially enabling processing over micro- to millisecond time scales. At first glance this stability against radiative decay might seem surprising given the large transition matrix elements between adjacent Rydberg states, which scale as $n^{2}$. However, spontaneous decay to nearby levels via low-frequency emission is strongly suppressed by the $\omega^{3}$ dependence of the Einstein $A$ coefficient. As a result, the predominant decay path for isolated Rydberg atoms in low-angular-momentum states is to the ground or low-lying excited levels, resulting in an $n^{-3}$ scaling of the spontaneous emission rate.

That said, neighboring Rydberg levels can play a dominant role in the decay of a large number $N$ of atoms which either are simultaneously excited in a volume with dimensions smaller than the wavelength $\lambda$ of the emitted light, or are sequentially excited throughout a cylindrical volume with length $L \gg \lambda$ [36,37]. In his seminal paper [38], Dicke predicted that a dense collection of $N$ radiators, either in very close proximity or in a properly phased extended distribution, could develop spontaneous correlations and collectively emit radiation at rates greatly exceeding ("superradiance"), or much smaller than ("subradiance"), those of individuals in the sample. For the two-level systems considered by Dicke, correlations between a large number of emitting atoms can initiate collective superradiant emission at a per atom rate up to N/4 times larger than that between the same 
CHAPTER 4. DECAY IN A COLD RYDBERG GAS

two levels in an isolated atom [38]. Collective emission remains a subject of considerable interest in many different contexts, including Rydberg atoms [39-51].

The presence of blackbody radiation, the existence of multiple photo decay channels in a Rydberg ladder, and strong DD interactions between atoms, all explicitly neglected in Dicke's original paper (and in many subsequent treatments) $[38,52,53]$, make it much more difficult to observe, characterize, and quantitatively predict collective decay phenomena in Rydberg gases. In particular, DD interactions can suppress superradiance. In a thermal gas, this suppression results from DD-mediated collisions that homogeneously dephase the individual emitters in the ensemble at a rate greater than the superradiance rate [50]. In a frozen gas, DD exchange interactions couple pairs, or larger groups, of atoms leading to a variation in transition energies across the ensemble. Such inhomogeneities squelch the correlations that underlie superradiance, along with the collective emission [47]. That said, a clear signature of superradiance between Rydberg states, a fluorescence cascade from a Rydberg ladder proceeding at a rate much greater than spontaneous emission of isolated atoms, was first observed following pulsed-laser excitation of an elongated volume $(L \gg \lambda)$ in a thermal cell [36]. More recently, direct evidence for superradiance was found in the millimeter-wave emission from a large cylindrical volume of Ca Rydberg atoms in a supersonic expansion, also with $L \gg \lambda[49,50]$. As pointed out in the latter work, the rates for superradiant decay and DD dephasing within a given decay channel are essentially identical, up to a multiplicative geometric factor $L / \lambda$ in the superradiance rate formula. Accordingly, it was suggested that collective decay should not play a major role in Rydberg depopulation unless $L \gg \lambda[50]$.

Still, despite competing DD effects, under certain conditions superradiance should play some role in cold ensembles where the dimensions of the excited volume are less than or comparable to $\lambda$. Indeed, evidence of reduced Rydberg lifetimes has been reported in several such experiments $[39,48,51,54,55]$. For example, Feng et al. observed a density- 
CHAPTER 4. DECAY IN A COLD RYDBERG GAS

dependent lifetime suppression of Cs Rydberg atoms in a magneto-optical trap (MOT) [54]. They attribute the suppression to a combination of neutral Rydberg atom collisions and superradiance. However, the evidence for superradiance appears tenuous as their calculations with and without superradiant contributions both fall within their measurement uncertainty (see their Fig. 2). In addition, if one applies their values for Rydberg collision velocity and cross section, the collisional depopulation rates are over three orders of magnitude too small to account for their observations. Han and Maeda attributed population transfer from initial to neighboring Rb Rydberg states to superradiance, but provided no evidence ruling out other possible population transfer mechanisms [56]. In other measurements, using fluorescence detection, Day et al. found Rydberg depopulation rates that were roughly twice that expected from single-atom spontaneous emission over a range of $n$ states and at low densities, $\rho \sim 1 \times 10^{7} \mathrm{~cm}^{-3}$ [48]. The small variation of the lifetime suppression with principal quantum number coupled with trap loss measurements argued against collisional depopulation and blackbody ionization. Instead, the enhanced Rydberg decay rate was found to be qualitatively consistent with a simplified collective decay model. In other experiments, the inclusion of superradiant decay channels was found to improve the quality of model fits to electromagnetically induced transparency measurements in cold Rydberg gases $[51,55]$.

Certainly, a substantial decrease in Rydberg lifetimes due to collective emission would have a significant impact on most cold Rydberg atom experiments. Perhaps more important, in the context of the exploration and control of few- or many-body Rydberg dynamics, are the influences of the spontaneous quantum correlations that are predicted to develop with the emission of the first photon from the sample and evolve as the Rydberg population descends through a ladder of Dicke states [38,53]. Interestingly, Wang et al. presented a sophisticated theoretical treatment of photo decay in a multilevel Rydberg system which makes definite predictions as to whether superradiance should occur for a given initial 
principal quantum number, atom density, and experimental volume [39]. The theory apparently reproduced the rapid decay, at a rate approximately 40 times greater than predicted from spontaneous emission alone, of an initial population of $43 p$ atoms in a MOT

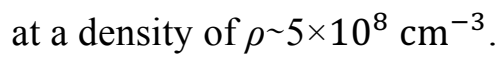

We have used pulsed-laser excitation of Rb Rydberg atoms in a MOT under conditions ostensibly similar to those used in Ref. [39] in an attempt to test the predictions of their Rydberg superradiance theory. We employ state-selective field ionization (SSFI) to measure the population in the initial and neighboring Rydberg states as a function of delay after the laser excitation. We find no evidence for the predicted collective decay over a range of principal quantum numbers $26 \leq n \leq 40$, and atom densities $\rho \sim 3 \times 10^{9} \mathrm{~cm}^{-3}$, despite the fact that, for these states, our highest density is more than two orders of magnitude above the predicted superradiance threshold [39]. Instead, our measurements are consistent with noncorrelated spontaneous decay combined with population redistribution via blackbody radiation.

\subsection{Experimental Procedure and Results}

General information about the setup could be found in Chapter 2. This section provides some particular information about the experiment approach.

In the experiments, ${ }^{85} R b$ atoms at $70 \mu \mathrm{K}$ are held in a MOT. The full width at half maximum (FWHM) diameter of the atom cloud is $0.4 \mathrm{~mm}$. The MOT is positioned at the center of four parallel rods which facilitate the application of static and pulsed electric fields in the y direction for exciting and detecting Rydberg atoms in the MOT. A 10 ns pulsed, tunable, dye-amplified, $\sim 480 \mathrm{~nm}$ diode laser propagating in the $\mathrm{x}$ direction is focused into the center of the MOT, creating a cylindrically shaped volume of cold Rydberg atoms with a FWHM diameter of $\sim 0.1 \mathrm{~mm}$ and a length of $0.4 \mathrm{~mm}$. The MOT and Rydberg lasers are 
CHAPTER 4. DECAY IN A COLD RYDBERG GAS

non collinear, preventing the excitation of Rydberg atoms throughout any extended volume from the lower-density background of thermal $\mathrm{Rb}$ atoms in the chamber. The $\sim 100 \mathrm{MHz}$ bandwidth of the Rydberg excitation laser ensures that there is no excitation suppression via dipole blockade [2,3]. At a variable time $\tau$ after the laser excitation, a ramped voltage is applied to two of the rods, ionizing any Rydberg atoms in the interaction region and pushing the resulting ions toward a micro channel plate (MCP) detector. Ions originating from different Rydberg states arrive at the detector at different times. The integrated signals in different time bins are proportional to the populations in different Rydberg states and are recorded for each laser shot as a function of the ionization time $\tau$. The experiment proceeds at the $15 \mathrm{~Hz}$ dye-laser repetition rate.

The diode laser is tuned to selectively excite atoms from the upper $5 p$ trap level to $n s$ and $n p$ Rydberg states with $26 \leq n \leq 40$. Excitation of $n p$ states is facilitated by the application of a weak static electric field (from $30 \mathrm{~V} / \mathrm{cm}$ at $n=26,16 \mathrm{~V} / \mathrm{cm}$ at $n=32$, to 7 $\mathrm{V} / \mathrm{cm}$ at $n=40$ ). By saturating the Rydberg excitation using high laser fluence, we ensure that approximately $50 \%$ of the $5 p$ atoms within the interaction volume are excited to Rydberg states, enabling us to determine the Rydberg atom density [57]. Subsidiary experiments on resonant energy transfer between Rydberg atoms are consistent with the Rydberg density determination [57,58]. The MOT fluorescence is monitored throughout the lifetime measurements, ensuring that the number of atoms in the MOT is constant to within a few percent as $\tau$ is scanned. Care is taken to minimize the amplified spontaneous emission from the dye-amplified laser pulse, eliminating direct photoionization of $5 p$ atoms. For the $s$-state measurements, a small, $\sim 1.5 \mathrm{~V} / \mathrm{cm}$, residual electric field persists in the interaction region due to imperfect shielding of the high voltage biased MCP (a larger field is present for initial $p$ states). This field is sufficient to eject any ions or electrons from the interaction region, eliminating extended interactions between charged particles and neutral Rydberg atoms, and preventing the spontaneous evolution of the Rydberg gas into a plasma 
CHAPTER 4. DECAY IN A COLD RYDBERG GAS

$[59,60]$. Neither the small static field employed for the $p$-state measurements nor the smaller residual field present during the $s$-state measurements substantially alters the rates for spontaneous emission or population transfer induced by blackbody radiation. The potential influence of the field inhomogeneity on superradiant decay is considered in the Analysis and Discussion section below.

Figure 4.1 and Figure 4.2 show our principal experimental results. In Figure 4.1 (a) and (c), the probabilities for finding atoms in the $26 s+25 p, 32 s$, and $40 s$ states are plotted as functions of detection time $\tau$ for the maximum densities explored, $\rho \sim 3 \times 10^{9} \mathrm{~cm}^{-3}$ and $\rho$ $\sim 1.5 \times 10^{9} \mathrm{~cm}^{-3}$, respectively. Note that for the lowest initial $n$ state the sum of the $26 \mathrm{~s}$ and $25 p$ populations is shown since their corresponding features could not be adequately separated in the time-resolved field-ionization signal. Within experimental uncertainties, the decays for the three initial $s$ states are identical at the two densities shown. Additional measurements were made at Rydberg densities as low as $\rho \sim 2 \times 10^{8} \mathrm{~cm}^{-3}$ (for $32 \mathrm{~s}$ initial states) and $\rho \sim 5 \times 10^{8} \mathrm{~cm}^{-3}$ (for $32 p$ initial states), but no statistically significant differences were observed in the decays.

For spontaneous decay of isolated atoms at absolute zero, one would expect lifetimes of 28 and $58 \mu$ s for the $32 s$ and $40 s$ atoms, respectively [61]. The measured lifetimes for the $32 s$ and $40 s$ states are substantially smaller, 19 and $38 \mu$ s, respectively, due to population redistribution by blackbody radiation from the $300 \mathrm{~K}$ environment surrounding the MOT. Indeed, redistributed population is detected in neighboring Rydberg levels. In particular, Figure 4.1 (b) and (d) show the delay-dependent population in the $p$ states $(26 p, 32 p$, and $40 p$ ) that lie immediately above the respective initial s states. Although we would expect to find some atoms in the adjacent, lower-lying $p$ states as well, small features reflecting that population in the time-of-ionization signal lie within the initial-state peak (for the case of $26 s$ ) or are masked by the tail of the larger, initial-state peak which precedes it. 

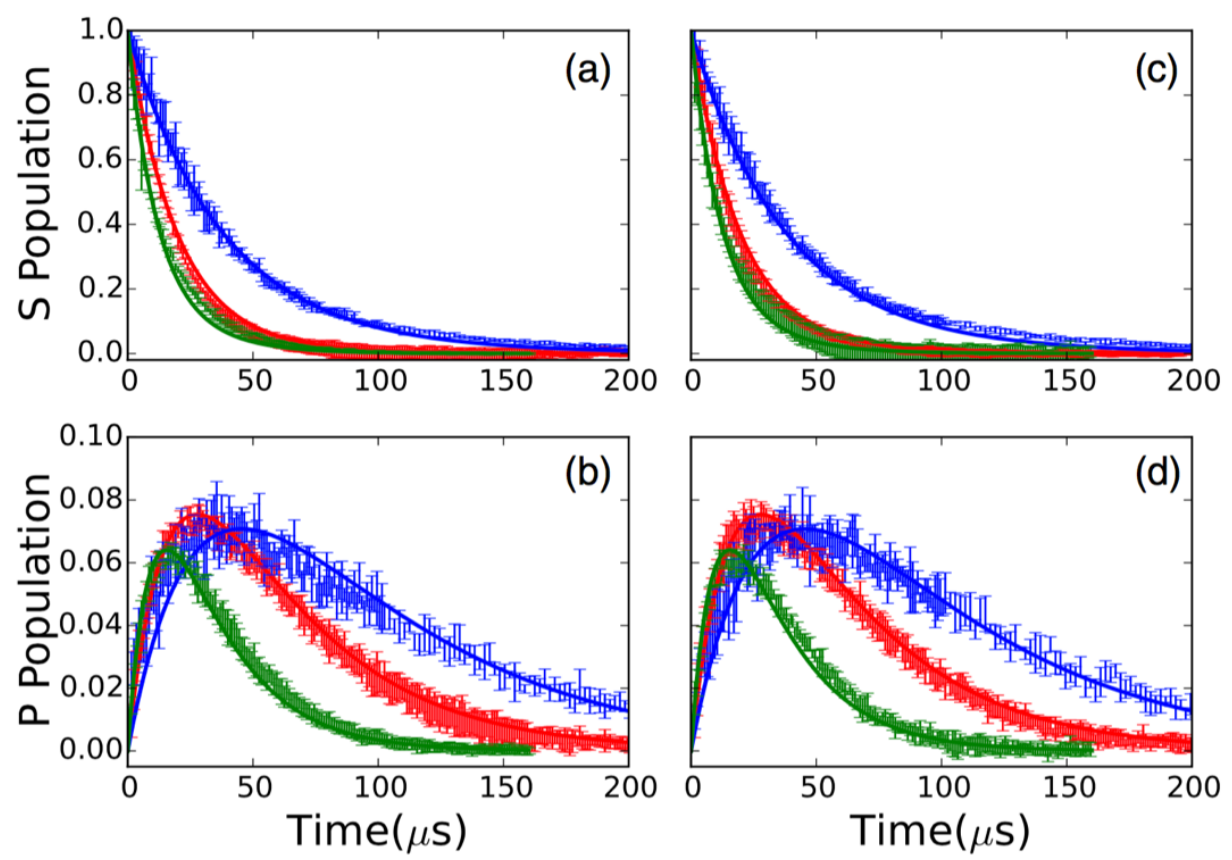

Figure 4.1: (a), (c) Probabilities for finding atoms in $26 s+25 p$ (green, fastest decay), $32 s$ (red, intermediate decay), and $40 s$ (blue, slowest decay) as a function of detection time $\tau$ for Rydberg densities of $\rho \sim 3 \times 10^{9} \mathrm{~cm}^{-3}$ (a) and $\rho \sim 1.5 \times 10^{8} \mathrm{~cm}^{-3}$ (c). Note that the sum of the $26 \mathrm{~s}$ and $25 \mathrm{p}$ populations is shown since their corresponding features could not be adequately separated in the field-ionization signal. Vertical bars show the experimental data with uncertainties, and the solid curves are calculated as described in the text. Measurements and calculations for the $40 s$ decay extend to $500 \mu$ s where the remaining population is negligible. (b), (d) Probabilities for finding atoms in $26 p$ (green, fastest rise and decay), $32 p$ (red, intermediate rise and decay), and 40p (blue, slowest rise and decay) levels as a function of detection time $\tau$. The states are populated by blackbody redistribution from the initial $26 s, 32 s$, and $40 s$ levels, respectively. The data were measured simultaneously with those shown in (a) and (c). Vertical bars show the experimental data with uncertainties, and the solid curves are calculated as described in the text. The measured $p$-state probabilities are normalized to the calculations as described in the text. The calculations have no free parameters and consider only the effects of spontaneous emission and blackbody radiation on isolated atoms.

The measured lifetime for the combined $26 s+25 p$ states is $14 \mu \mathrm{s}$, the same as that expected from spontaneous decay of the 26s level alone [61]. Simulations (described in detail below) indicate that this apparent agreement is not due to the absence of blackbody transfer out of $26 \mathrm{~s}$. Rather, the small longer-lived $25 \mathrm{p}$ component of the signal masks much of the change in the $26 s$ decay, with a predicted effective lifetime of $13 \mu$ s for the $26 s+25 p$ combination, similar to what we observe. We note that due to the slow rate of the ionizing field, there is a distribution of ionization times and, therefore, of detection efficiencies for 
atoms in different states. As a result, each of the measured $p$-state populations in Figure 4.1 (b) and (d) has been multiplied by a normalization factor to obtain the best agreement with the calculated decay curves that are shown in the figures and described in the next section.

Figure 4.2 shows analogous data for the decay of initially excited $26 p, 32 p$, and $40 p$ states. Again, due to blackbody redistribution, the lifetimes associated with these decays $(18,31$, and $51 \mu \mathrm{s})$, are considerably smaller than expected from spontaneous emission alone $(37,75$, and $155 \mu \mathrm{s})[61]$. However, in this case, no substantial population is detected in the neighboring $s$ or $d$ levels. The analysis described in the next section indicates that the populations in these states are not detectable within our signal-to-noise ratio, remaining at or below the few percent level due to the relatively rapid spontaneous emission rate out of the s states, and smaller $p \rightarrow s$ and $p \rightarrow d$ blackbody transition rates.
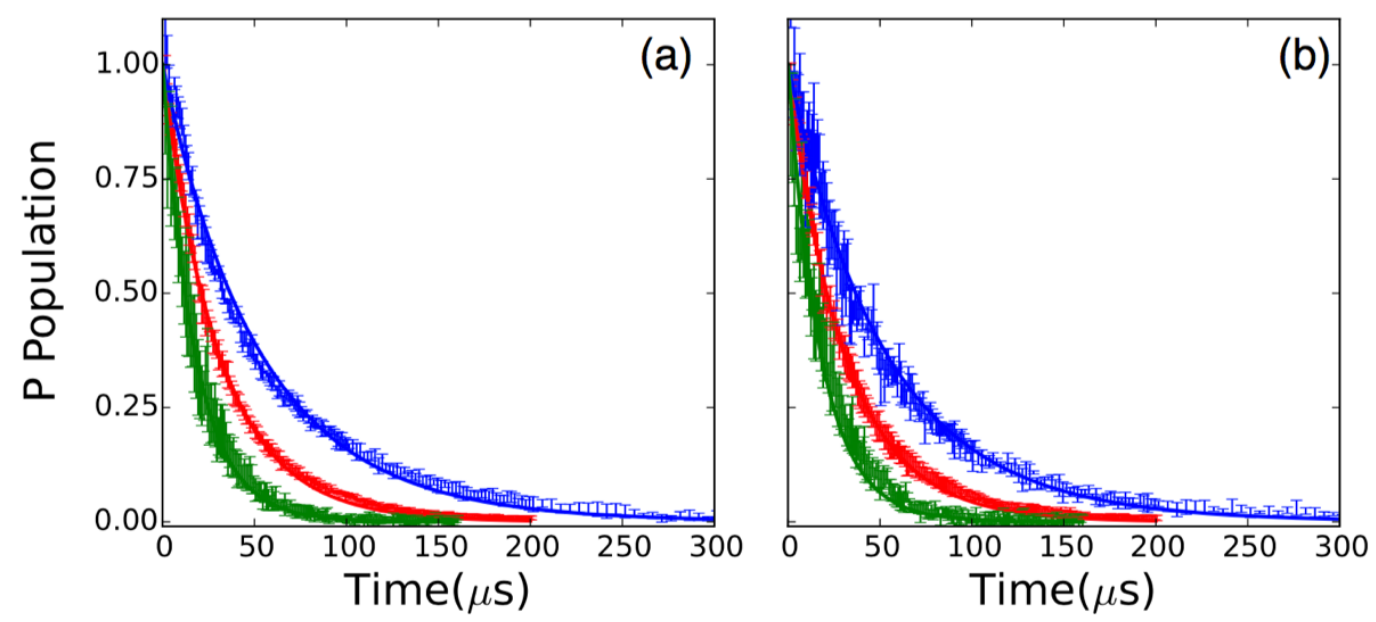

Figure 4.2: Probabilities for finding atoms in $26 p$ (green, fastest decay), $32 p$ (red, intermediate decay), and $40 p$ (blue, slowest decay) as functions of detection time $\tau$ for Rydberg densities of $\rho \sim 3 \times 10^{9} \mathrm{~cm}^{-3}$ (a) and $\rho \sim 1.5 \times 10^{9} \mathrm{~cm}^{-3}$ (b). Vertical bars show the experimental data with uncertainties, and the solid curves are calculated as described in the text. Measurements and calculations for the $40 p$ decay extend to 500 $\mu$ s where the remaining population is negligible. The calculations have no free parameters and consider only the effects of spontaneous emission and blackbody radiation on isolated atoms. 


\subsection{Analysis and Discussion}

To determine if collective processes play any significant role in the decays we observe, we compare the measurements to the results of a simple rate equation model which has an example schematic shown in Figure 3.6. The model includes population transfer via stimulated emission and absorption of blackbody radiation between an essential set of $s, p$, and $d$ Rydberg states neighboring the initial level, as well as spontaneous emission out of those essential states to (undetected) lower-lying levels. We calculate the blackbody transition rates between the essential states [1] as well as the known total spontaneous emission rates of the $s, p$, and $d$ Rydberg levels.

For example, for an initially excited $40 s$ state, the rate equation describing the timedependent population in the initial $40 \mathrm{~s}$ level is:

$$
\begin{aligned}
\frac{d N_{40 s}}{d t}= & \left(-A_{40 s}-B_{40 s \rightarrow 40 p}-B_{40 s \rightarrow 39 p}\right) N_{40 s} \\
& +B_{39 p \rightarrow 40 s} N_{39 p}+B_{40 p \rightarrow 40 s} N_{40 p}
\end{aligned}
$$

where $N_{n \ell}$ is the population in state $n \ell, A_{40 s}$ is the $40 s$ spontaneous decay rate, and $B_{n \ell \rightarrow n^{\prime} \ell^{\prime}}$ is the blackbody transition rate from $n \ell$ to $n^{\prime} \ell^{\prime}$ :

$$
B_{n^{\prime} \ell^{\prime}, n \ell}=2 \bar{n} \alpha^{3} w_{n^{\prime} \ell^{\prime}, n \ell}^{2}\left|\bar{f}_{n^{\prime} \ell^{\prime}, n \ell}\right|
$$

which is mentioned in Chapter 3. We use the spontaneous emission rates calculated by Gounnad [61].

The populations in the secondary states $39 p$ and $40 p$ are computed using similar rate equations that include the total spontaneous decay rate out of those levels as well as blackbody transitions to and from pairs of $s$ and $d$ levels that lie immediately above and below each $p$ state. We truncate the system of equations with rate equations that include spontaneous decay from the tertiary $s$ and $d$ levels and their blackbody couplings with the secondary states. Analogous systems of equations are used to compute the Rydberg 
CHAPTER 4. DECAY IN A COLD RYDBERG GAS

population decay following initial $p$-state excitation. We note that, for initial or intermediate $p$ states in particular, blackbody radiation redistributes a small, but nonnegligible, fraction of the initial population beyond the nearest-neighbor $s$ and $d$ states. Therefore, an approximate expression [1]

$$
B_{n \ell}=\frac{4 \alpha^{3} k T}{3 n^{2}}
$$

for the total blackbody decay rate from each $p$ level is used to more accurately account for the net transfer out of these states.

The results of our calculation, which ignore any collective decay phenomena, are shown with the data in Figure 4.1. Overall, the agreement is reasonable. Aside from the previously noted renormalization of the experimental $p$-state population, no parameter adjustments have been made to obtain the level of agreement shown. The data provide no evidence of a significant reduction in the Rydberg lifetimes due to superradiance. This is true over a range of principal quantum numbers and atom densities where superradiant emission has been predicted to be the dominant decay path [39].

It is well established that superradiance is suppressed by inhomogeneities in transition energies across a sample of emitters [47], and we suspect that this is the case in our, and many other, cold atom experiments. In our experiments, three different effects contribute to such inhomogeneities. The first, and dominant mechanism for some of our measurements, is the DD exchange interaction. Consider a pair of identical atoms with two levels $s$ and $p$ and interatomic separation $R$. Spontaneous emission from the initial upper pair state $s S$ results in the population of the bright configuration of the lower-energy pair state $(s p+$ $p s) / \sqrt{2}$. However, due to the DD coupling between the atoms, $V_{D D} \propto|\langle s|r| p\rangle|^{2} / R^{3}$, the energy of this state is not the same as that for two atoms at infinite separation [1]. Accordingly, in a large ensemble of randomly spaced atoms, every possible configuration of $N_{s} s$ atoms and $N_{p} p$ atoms has a different energy, depending on the separation (and 
relative orientation) between the $p$ atoms and their neighboring $s$ atoms. As a result, any Dicke state, the bright linear combination of all possible configurations of $N_{s} s$ atoms and $N_{p} p$ atoms [38], is nonstationary. The phases of the constituent $N$-atom product states evolve at different rates, as determined by their DD energy shifts relative to their energies at infinite separation. The emission from these nonstationary Dicke states dephases at a rate comparable to the typical dipole-dipole energy shift $V_{D D}$ for pairs of atoms in the ensemble. Superradiance cannot occur unless the system transitions down each step in the Dicke ladder more rapidly than this dephasing. A similar argument has been made by Gross and Haroche [62]. In the frequency domain, atoms with different transition energies at different locations in the ensemble do not collectively emit into the same field unless that emission occurs in a very short burst with a sufficiently broad, coherent bandwidth.

To determine the DD dephasing rate, we use the most probable nearest-neighbor separation in a random ensemble as we mentioned in 3.2.2, $R \simeq(2 \pi \rho)^{-3}$, and average over all orientations of the Rydberg states on any two neighboring atoms $n s$ and $n^{\prime} p$ to obtain $[63,64]:$

$$
\overline{V_{D D}}=\frac{8 \pi}{9} \rho|<n s| r\left|n^{\prime} p>\right|^{2}
$$

Using a numerical Numerov integration algorithm to compute the relevant radial matrix elements [65], at the highest density studied $\left(\rho=3 \times 10^{9} \mathrm{~cm}^{-3}\right)$ we obtain values for the DD exchange coupling between the $n s$ and $(n-1) p$ states, $\overline{V_{D D}}=2.4,6.2$, and $17 \mathrm{MHz}$, for $n=26,32$, and 40 , respectively. These interaction strengths set effective lower limits for the rates at which collective emission from $n s$ to $(n-1) p$ can occur. Similarly, for initial $n p$ states and $\rho=3 \times 10^{9} \mathrm{~cm}^{-3}$, the relevant exchange coupling is to the nearest lowerlying $s$ states, with $, \overline{V_{D D}}=3.1,7.8$, and $20 \mathrm{MHz}$, for $n=26,32$, and 40, respectively.

The magnetic field gradient in the MOT is another source of energy inhomogeneities in our ensemble. As in Ref. [39], the magnetic field remains on during our measurements, 
resulting in a transition energy variation of approximately $1 \mathrm{MHz}$ across the MOT. This inhomogeneity is smaller, or much smaller, than that due to dipole-dipole interactions at sufficiently high densities. It should not play a principal role in suppressing superradiance under the conditions used to produce Figure 4.1 and Figure 4.2.

The third contributor to the Rydberg energy variations across the ensemble is electric field inhomogeneity. While the voltages applied to the field rods produce a field that is quite uniform over the MOT (predicted field variations of $0.07 \%$, corresponding to 21 $\mathrm{mV} / \mathrm{cm}$ for the largest applied field of $30 \mathrm{~V} / \mathrm{cm}$ for the $26 p$ measurements) the residual field from the MCP is not as uniform. Using a combination of spectroscopic measurements and accurate Stark energy calculations, we determine an upper limit for the Rydberg energy inhomogeneity due to the nonuniformity of the electric field $F$ in the interaction region.

First, we measure the transition frequencies for excitation of $32 p_{3 / 2}\left|m_{j}\right|=1 / 2,3 / 2$, from the $5 p_{3 / 2}$ upper trap state as a function of the voltage applied to the field rods (see Figure 4.3). For convenience, in the following discussion we refer to the field produced by the rods as the "applied" field. The experimental geometry is identical to that used for the lifetime measurements, but the Rydberg excitation is performed with an unamplified, $3 \mu \mathrm{s}$ pulse chopped from the $\sim 1 \mathrm{MHz}$ bandwidth cw diode laser. The Rydberg excitation pulse has $\sim 1 \mu$ s rise and fall times and is formed using an acousto-optic modulator. We use a temperature- and pressure-stabilized Fabry-Pérot interferometer to track the relative frequency of the Rydberg laser as it is scanned. The population in $\left|m_{j}\right|=1 / 2$ is distinguished from that in $\left|m_{j}\right|=3 / 2$ using SSFI. By recording the signal in two different time bins we obtain (nominally) separate excitation profiles to the two $\left|m_{j}\right|$ states in the same laser frequency scan. Therefore, the energy splitting between the two $m_{j}$ states can be accurately determined to well within the excitation bandwidth which is dominated by the $6.07 \mathrm{MHz}$ natural linewidth of the initial $5 p_{3 / 2}$ level. 


\section{CHAPTER 4. DECAY IN A COLD RYDBERG GAS}

In zero electric field, the excitation profiles associated with the population in the two $\left|m_{j}\right|$ levels should exhibit maxima at the same laser frequency, i.e., have zero energy splitting. However, as shown in Figure 4.4, we observe a minimum splitting of $2 \mathrm{MHz}$ at an applied field of $-2.8 \mathrm{~V} / \mathrm{cm}$. The minimum splitting at nonzero applied field allows us to determine the components of the MCP field parallel and perpendicular to applied field. Apparently, the application of a $-2.8 \mathrm{~V} / \mathrm{cm}$ rod field minimizes the net field in the interaction region. Accordingly, there must be a parallel, $2.8 \mathrm{~V} / \mathrm{cm}$, MCP field component which we call the "offset" field. Using the variation in the $\left|m_{j}\right|$ splitting as a function of applied field, we can also extract a value, $1.5 \mathrm{~V} / \mathrm{cm}$, for the perpendicular, i.e., "residual," MCP field component. The solid curve shown with the data in Figure 4.4 is the predicted $32 p_{3 / 2}\left|m_{j}\right|=1 / 2,3 / 2$ splitting as a function of applied field (extracted from a full numerical Stark map calculation based on the method of Zimmerman et al. [65]), assuming MCP offset and residual fields of 2.8 and $1.5 \mathrm{~V} / \mathrm{cm}$, respectively. The good agreement with experiment confirms the accuracy of the calculation as well as the offset and residual field determinations.

At, and near, the minimum splitting (i.e., in the presence of the residual field alone where the $s$-state decay measurements are performed), the $\left|m_{j}\right|$ excitation resonances have minimum linewidths of $8 \mathrm{MHz}$ (see Figure 4.3). As noted above, the predominant contribution to this linewidth is the $6.07 \mathrm{MHz}$ natural width of the $5 p_{3 / 2}$ level. However, the laser bandwidth, Zeeman shifts due to magnetic field inhomogeneities, and Stark shifts due to inhomogeneities in the $1.5 \mathrm{~V} / \mathrm{cm}$ residual field also contribute. Assuming that the laser spectrum and field distributions are Gaussian, we deconvolute the primary line shape as a Voigt profile, and extract a bandwidth of $3.9 \mathrm{MHz}$ for the total Gaussian contribution. Accordingly, we obtain an upper-limit estimate for the electric field inhomogeneity by assuming it is the sole contributor to this width. From the Stark shift of the $32 p_{3 / 2} m_{j}=1 / 2$ level, $E=6.5 \mathrm{MHz} /(\mathrm{V} / \mathrm{cm})^{2} F^{2}$, we determine that the maximum possible variation of the 
residual field across the interaction region is $F_{r e s}=0.20 \mathrm{~V} / \mathrm{cm}$. Using this field inhomogeneity with the field-dependent Stark shifts of the respective levels, we can compute the maximum range of transition energies between the initial $s$ states and the $p$ states immediately below them (to which the dipole coupling is the strongest). For the $26 \mathrm{~s}$ $\rightarrow 25 p, 32 s \rightarrow 31 p$, and $40 s \rightarrow 39 p$ transitions, the maximum energy variations across the excitation region (with only the residual field present) are $0.43,2.2$, and $12 \mathrm{MHz}$, respectively. The transition energy variations are smaller for transitions to lower lying $p$ states due to the $n^{7}$ scaling of the Rydberg polarizability. So, at the highest densities we have explored, the energy inhomogeneities associated with the residual electric field are less, or much less, than those associated with the dipole-dipole exchange interaction. Therefore, the electric field inhomogeneities do not hold the primary responsibility for the suppression of superradiance from any of the initial $s$ states. 


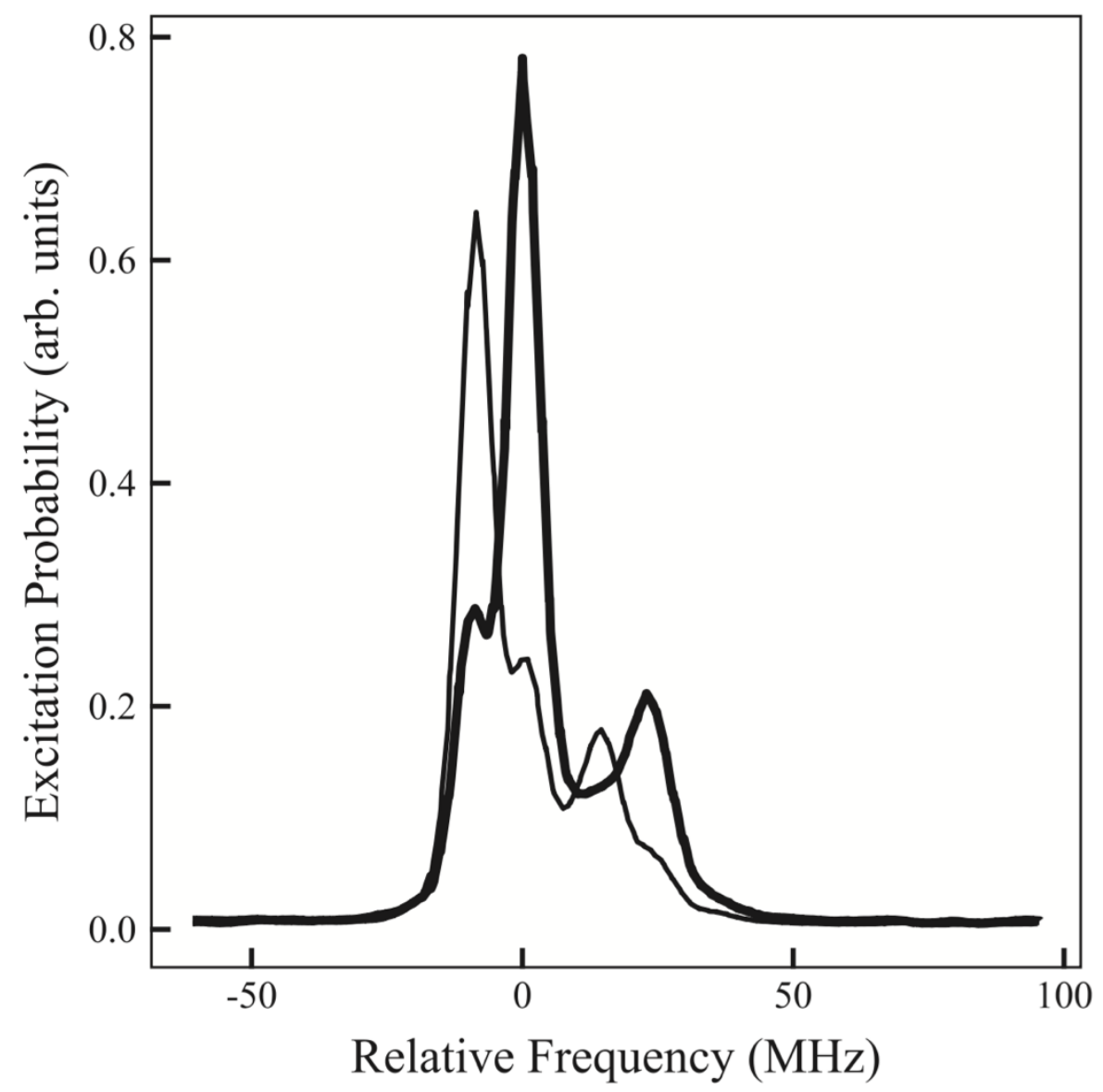

Figure 4.3: Measured $32 p_{3 / 2}\left|m_{j}\right|=1 / 2$ (bold line) and $\left|m_{j}\right|=3 / 2$ (thin line) excitation probabilities as a function of Rydberg laser frequency in zero applied field. The two data curves are obtained simultaneously in the same laser frequency scan. The small feature on the left (right) of the main $\left|m_{j}\right|=1 / 2(3 / 2)$ peak is the result of imperfect discrimination of the $\left|m_{j}\right|-1 / 2$ and $3 / 2$ components via SSFI. The additional peak on the right of the main feature in each trace is due to the trap-laser dressing of the $5 p_{3 / 2}$ and $5 s$ levels. Its frequency shift from the main peak reflects the Autler-Townes splitting of the $5 p_{3 / 2}$ initial state. 


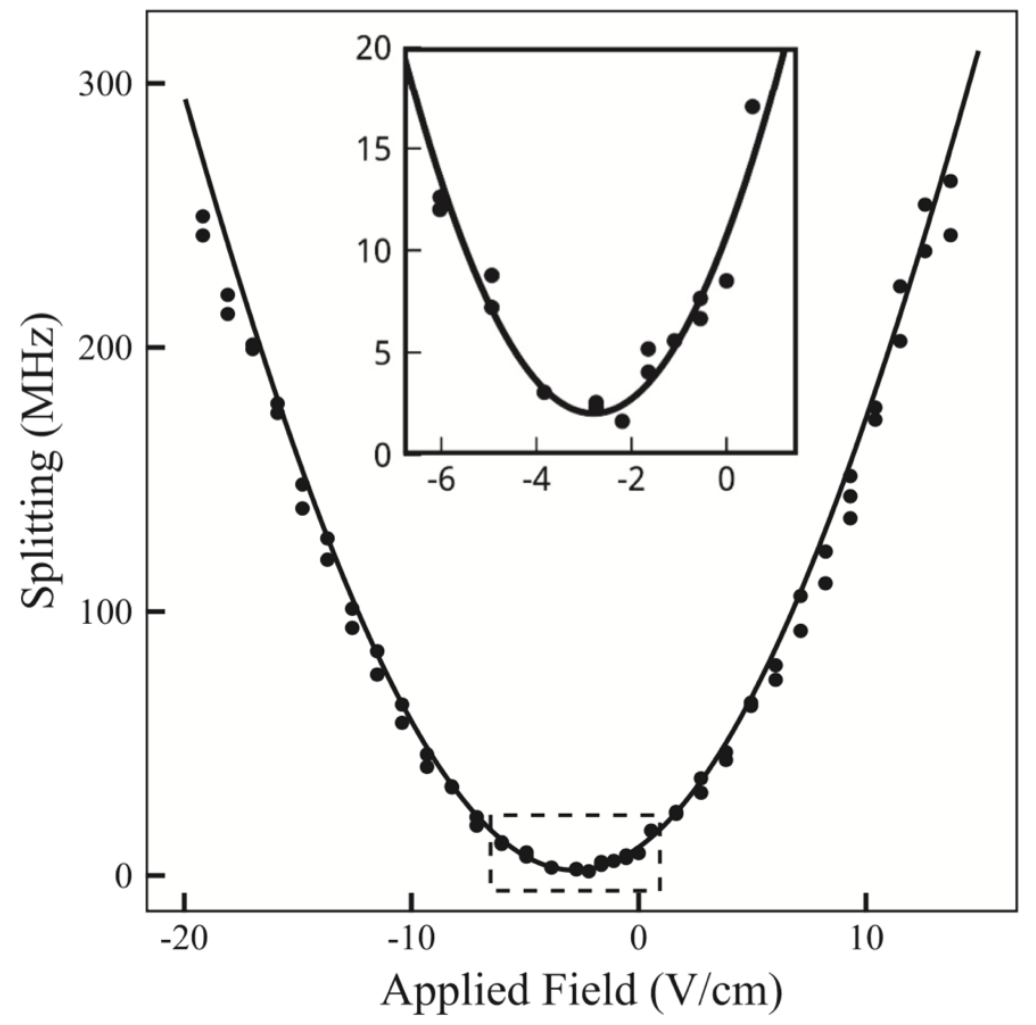

Figure 4.4: Difference (i.e., splitting) in the transition energies for exciting $32 p_{3 / 2}$ $\left|m_{j}\right|=1 / 2,3 / 2$ from $5 p_{3 / 2}$ as a function of applied electric field. Filled circles are measurements and the solid curve is the result of a numerical Stark map calculation assuming orthogonal "offset" and "residual" electric field components due to the $\mathrm{MCP}$ of 2.8 and $1.5 \mathrm{~V} / \mathrm{cm}$, respectively. The inset shows a magnified view of the portion of the main figure within the dashed window.

The situation with the initial $p$ states is somewhat different, as they are excited in a nonzero applied field that is considerably larger than the orthogonal residual field. As a result, the residual field and its inhomogeneity have essentially no effect on the transition energies. However, the spatial variations in the MCP offset field, which is parallel to the applied field, cannot be neglected. We use measurements of DD-mediated resonant energy transfer between Rydberg atoms to obtain an upper-limit estimate for the MCP offset field inhomogeneity. Those experiments use the same experimental geometry as the Rydberg decay measurements $[58,64]$. In the experiments, the probability for resonant population transfer from one pair of Rydberg states to another (e.g., $25 s+33 s \rightarrow 24 p+34 p$ [64]) is recorded as a function of an applied field which Stark-tunes the total energies of the atom 
CHAPTER 4. DECAY IN A COLD RYDBERG GAS

pair in the two different configurations. In a uniform field, the line shape describing the field-dependent energy transfer probability is characterized by a peak at the "resonance" condition, where the total energies of the two sets of atom pair states are identical, and a width that is proportional to the Rydberg density. In a nonuniform field, the line shape has a nonzero minimum width as the density approaches zero, due to variations in the local field at different locations within the sample. Consider the $25 s+33 s \rightarrow 24 p+34 p$ resonance [64] for which maximum population transfer occurs in an electric field of $F$ $3.4 \mathrm{~V} / \mathrm{cm}$. Assuming that the nonzero resonance width that is observed at very low Rydberg density [64] is due solely to the inhomogeneity in the electric field (i.e., ignoring magnetic field inhomogeneities and any other broadening effects) we obtain the maximum possible variation in the offset field, $F_{\text {off }}=0.08 \mathrm{~V} / \mathrm{cm}$, across the Rydberg sample. As an additional check, we consider a different energy transfer resonance, $32 p+32 p \rightarrow 33 s+32 s$, that is centered at a substantially higher field $F \sim 11.5 \mathrm{~V} / \mathrm{cm}$ [58]. The nonzero low-density width for this energy transfer resonance gives the same maximum value for the offset field inhomogeneity, $F_{\text {off }}=0.08 \mathrm{~V} / \mathrm{cm}$.

Given $F_{o f f}$, we can compute the maximum possible variations in the energies, associated with transitions between initial $p$ states and the nearest lower-lying $s$ state, due to the inhomogeneous field. Using $F_{o f f}$, the calculated Stark shifts of each of the states involved in the transitions $26 p \rightarrow 26 s, 32 p \rightarrow 32 s$, and $40 p \rightarrow 40 s$, and the applied fields employed for the respective $p$-state excitations, we obtain the maximum possible transition energy variations due to the inhomogeneous electric field. These are 4.9, 13, and $30 \mathrm{MHz}$ for the $26 p, 32 p$, and $40 p$ initial states, respectively. Accordingly, for the $p$-state decays, the maximum energy variations due to the field are comparable to, but up to a factor of $1.7 \times$ larger than, those due to dipole-dipole interactions. Given our likely overestimate of 
CHAPTER 4. DECAY IN A COLD RYDBERG GAS

the field inhomogeneity, both may play a role in suppressing collective emission from the ensemble.

\subsection{Conclusion}

We have studied the decay of Rydberg excitations in a cold Rb gas and find no evidence for the dramatic decrease in lifetimes predicted by Wang et al. [39]. The decay rates and population redistribution we observe are consistent with a model that considers only spontaneous emission from, and blackbody redistribution within, isolated atoms. In our experiments, a small electric field in the interaction region ejects any free electrons or ions from the excitation volume, preventing ionization or population transfer due to interactions with charged particles. In addition, the lack of spatial overlap between the trapping lasers and the Rydberg excitation laser well outside of the cold atom cloud ensures that there is no Rydberg excitation within an extended volume of lower-density, background $\mathrm{Rb}$ atoms in the chamber. In the experiment Wang et al. [39], the excitation of such an extended volume may have enabled collective emission in the $L>>\lambda$ regime, resulting in the rapid depopulation that was observed. More generally, in the $L<\lambda$ regime, suppression of superradiant emission should occur due to variations in transition energies across the cold Rydberg atom sample. These energy variations, can be due to inhomogeneous fields and/or variations in the DD coupling between atoms throughout the ensemble. The latter of which will be present any time the separation between atoms is not fixed. In our measurements, for initial $s$ states, DD exchange interactions within the random ensemble dominate the inhomogeneities. For initial $p$ states, the suppression is likely due to a combination of DD exchange and electric field inhomogeneities. 


\section{Bibliography}

[1] Thomas F. Gallagher. Rydberg Atoms. Cambridge University Press (1994).

[2] M. D. Lukin, M. Fleischhauer, R. Cote, L. M. Duan, D. Jaksch, J. I. Cirac, and P. Zoller, Phys. Rev. Lett. 87, 037901 (2001).

[3] D. Tong, S. M. Farooqi, J. Stanojevic, S. Krishnan, Y. P. Zhang, R. Cote, E. E. Eyler, and P. L. Gould, Phys. Rev. Lett. 93, 063001 (2004).

[4] F. Robicheaux and J. V. Hernandez, Phys. Rev. A 72, 063403 (2005).

[5] T. Cubel Liebisch, A. Reinhard, P. R. Berman, and G. Raithel, Phys. Rev. Lett. 95, $253002(2005)$.

[6] T. Vogt, M. Viteau, J. Zhao, A. Chotia, D. Comparat, and P. Pillet, Phys. Rev. Lett. 97, $083003(2006)$.

[7] E. Urban, T. A. Johnson, T. Henage, L. Isenhower, D. D. Yavuz, T. G. Walker, and M. Saffman, Nat. Phys. 5, 110 (2009).

[8] Alpha Gaëtan, Yevhen Miroshnychenko, Tatjana Wilk, Amodsen Chotia, Matthieu Viteau, Daniel Comparat, Pierre Pillet, Antoine Browaeys, and Philippe Grangier, Nat. Phys. 5, 115 (2009).

[9] T. Wilk, A. Gaetan, C. Evellin, J. Wolters, Y. Miroshnychenko, P. Grangier, and A. Browaeys, Phys. Rev. Lett. 104, 010502 (2010).

[10] Hendrik Weimer, Robert Low, Tilman Pfau, and Hans Peter Buchler, Phys. Rev. Lett. $101,250601(2008)$

[11] M. Saffman and K. Molmer, Phys. Rev. Lett. 102, 240502 (2009).

[12] Robert Low, Hendrik Weimer, Ulrich Krohn, Rolf Heidemann, Vera Bendkowsky, Bjorn Butscher, Hans Peter Buchler, and Tilman Pfau, Phys. Rev. A 80, 033422 (2009). 
[13] T. Pohl and P. R. Berman, Phys. Rev. Lett. 102, 013004 (2009).

[14] Thomas Amthor, Christian Giese, Christoph S. Hofmann, and Matthias Weidemuller, Phys. Rev. Lett. 104, 013001 (2010).

[15] T. Pohl, E. Demler, and M. D. Lukin, Phys. Rev. Lett. 104, 043002 (2010).

[16] S. Wuster, C. Ates, A. Eisfeld, and J. M. Rost, Phys. Rev. Lett. 105, 053004 (2010).

[17] Jens Honer, Hendrik Weimer, Tilman Pfau, and Hans Peter Buchler, Phys. Rev. Lett. $105,160404(2010)$.

[18] J. D. Pritchard, D. Maxwell, A. Gauguet, K. J. Weatherill, M. P. A. Jones, and C. S. Adams, Phys. Rev. Lett. 105, 193603 (2010).

[19] A. Schwarzkopf, R. E. Sapiro, and G. Raithel, Phys. Rev. Lett. 107, 103001 (2011).

[20] S. Sevincli, N. Henkel, C. Ates, and T. Pohl, Phys. Rev. Lett. 107, 153001 (2011).

[21] F. Bariani, Y. O. Dudin, T. A. B. Kennedy, and A. Kuzmich, Phys. Rev. Lett. 108, 030501 (2012).

[22] Matthieu Viteau, Paul Huillery, Mark G. Bason, Nicola Malossi, Donatella Ciampini, Oliver Morsch, Ennio Arimondo, Daniel Comparat, and Pierre Pillet, Phys. Rev. Lett. 109, 053002 (2012).

[23] D. D. Bhaktavatsala Rao and Klaus Molmer, Phys. Rev. Lett. 111, 033606 (2013).

[24] H. Schempp, G. Gunter, M. Robert-de-Saint-Vincent, C. S. Hofmann, D. Breyel, A. Komnik, D. W. Schonleber, M. Garttner, J. Evers, S. Whitlock, and M. Weidemuller, Phys. Rev. Lett. 112, 013002 (2014).

[25] Matthew Ebert, Alexander Gill, Michael Gibbons, Xianli Zhang, Mark Saffman, and Thad G. Walker, Phys. Rev. Lett. 112, 043602 (2014).

[26] D. Paredes-Barato and C. S. Adams, Phys. Rev. Lett. 112, 040501 (2014).

[27] H. Gorniaczyk, C. Tresp, J. Schmidt, H. Fedder, and S. Hofferberth, Phys. Rev. Lett. 113, $053601(2014)$. 
[28] Daniel Tiarks, Simon Baur, Katharina Schneider, Stephan Dürr, and Gerhard Rempe, Phys. Rev. Lett. 113, 053602 (2014).

[29] David Petrosyan and Klaus Molmer, Phys. Rev. Lett. 113, 123003 (2014).

[30] J. Pellegrino, R. Bourgain, S. Jennewein, Y. R. P. Sortais, A. Browaeys, S. D. Jenkins, and J. Ruostekoski, Phys. Rev. Lett. 113, 133602 (2014).

[31] Daniel Barredo, Henning Labuhn, Sylvain Ravets, Thierry Lahaye, Antoine Browaeys, and Charles S. Adams, Phys. Rev. Lett. 114, 113002 (2015).

[32] Y. O. Dudin and A. Kuzmich, Science 336, 887 (2012).

[33] Alexander W. Glaetzle, Marcello Dalmonte, Rejish Nath, Christian Gross, Immanuel Bloch, and Peter Zoller, Phys. Rev. Lett. 114, 173002 (2015).

[34] R. M. W. van Bijnen and T. Pohl, Phys. Rev. Lett. 114, 243002 (2015).

[35] M. Ebert, M. Kwon, T. G. Walker, and M. Saffman, Phys. Rev. Lett. 115, 093601 $(2015)$

[36] F. Gounand, M. Hugon, P. R. Fournier, and J. Berlande, J. Phys. B 12, 547 (1979).

[37] C. Carr, R. Ritter, C. G. Wade, C. S. Adams, and K. J. Weatherill, Phys. Rev. Lett. $111,113901(2013)$.

[38] R. H. Dicke, Phys. Rev. 93, 99 (1954).

[39] T. Wang, S. F. Yelin, R. Cote, E. E. Eyler, S. M. Farooqi, P. L. Gould, M. Kostrun, D. Tong, and D. Vrinceanu, Phys. Rev. A 75, 033802 (2007).

[40] N. Skribanowitz, I. P. Herman, J. C. MacGillivray, and M. S. Feld, Phys. Rev. Lett. 30, 309 (1973).

[41] M. Gross, C. Fabre, P. Pillet, and S. Haroche, Phys. Rev. Lett. 36, 1035 (1976).

[42] D. Pavolini, A. Crubellier, P. Pillet, L. Cabaret, and S. Liberman, Phys. Rev. Lett. 54, 1917 (1985).

[43] M. G. Moore and P. Meystre, Phys. Rev. Lett. 83, 5202 (1999).

[44] J. I. Kim, R. B. B. Santos, and P. Nussenzveig, Phys. Rev. Lett. 86, 1474 (2001). 
[45] C. Greiner, B. Boggs, and T. W. Mossberg, Phys. Rev. Lett. 85, 3793 (2000).

[46] Chiu Fan Lee and Neil F. Johnson, Phys. Rev. Lett. 93, 083001 (2004).

[47] Vasily V. Temnov and Ulrike Woggon, Phys. Rev. Lett. 95, 243602 (2005).

[48] J. O. Day, E. Brekke, and T. G. Walker, Phys. Rev. A 77, 052712 (2008).

[49] Kirill Prozument, Anthony P. Colombo, Yan Zhou, G. B. Park, Vladimir S. Petrovic, Stephen L. Coy, and Robert W. Field, Phys. Rev. Lett. 107, 143001 (2011).

[50] Anthony P. Colombo, Yan Zhou, Kirill Prozument, Stephen L. Coy, and Robert W. Field, J. Chem. Phys. 138, 014301 (2013).

[51] Florian Karlewski, Markus Mack, Jens Grimmel, Nora Sandor, and Jozsef Fortagh, Phys. Rev. A 91, 043422 (2015).

[52] N. E. Rehler and J. H. Eberly, Phys. Rev. A 3, 1735 (1971).

[53] R. Bonifacio and L. A. Lugiato, Phys. Rev. A 11, 1507 (1975).

[54] Zhi-Gang Feng, Lin-Jie Zhang, Jian-Ming Zhao, Chang-Yong Li, and Suo-Tang Jia, J. Phys. B 42, 145303 (2009).

[55] K. J. Weatherill, J. D. Pritchard, R. P. Abel, M. G. Bason, A. K. Mohapatra, and C. S. Adams, J. Phys. B 41, 201002 (2008).

[56] Jianing Han and H. Maeda, Can. J. Phys. 92, 1130 (2014).

[57] M. R. Kutteruf, Coherence in Rydberg atoms: Measurement and Control, Ph.D. dissertation, University of Virginia (2010).

[58] B. G. Richards and R. R. Jones (unpublished).

[59] Wenhui Li, Michael W. Noel, Michael P. Robinson, Paul J. Tanner, Thomas F. Gallagher, Daniel Comparat, Bruno Laburthe Tolra, Nicolas Vanhaecke, Thibault Vogt, Nassim Zahzam, Pierre Pillet, and Duncan A. Tate, Phys. Rev. A 70, 042713 (2004).

[60] M. P. Robinson, Interactions in a frozen Rydberg gas, Ph.D. dissertation, University of Virginia, 2002. 
[61] F. Gounand, J. Phys. (Paris) 40, 457 (1979).

[62] M. Gross and S. Haroche, Phys. Rep. 93, 301 (1982).

[63] M. R. Kutteruf and R. R. Jones, Phys. Rev. A 82, 063409 (2010).

[64] M. R. Kutteruf and R. R. Jones, Phys. Rev. Lett. 108, 013001 (2012).

[65] M. L. Zimmerman, M. G. Littman, M. M. Kash, and D. Kleppner, Phys. Rev. A 20, $2251(1979)$. 


\title{
5 Rydberg Wavepacket Evolution in A Frozen Gas of DD Coupled Atoms
}

\author{
Abstract \\ We have studied the evolution of Rydberg wavepackets in the presence of interatomic \\ dipole- dipole interactions in a frozen $\mathrm{Rb}$ gas. $\mathrm{Rb}$ atoms in a MOT are first laser-excited to \\ ns Rydberg eigenstates. A picosecond $\mathrm{THz}$ pulse further excites them into coherent \\ superposition states involving the initial-level and neighboring np-states. A second, \\ identical, time-delayed $\mathrm{THz}$ pulse probes the wavepacket dynamics. As the wavepackets \\ evolve they are influenced by dipole-dipole interactions, predominantly pairwise \\ excitation-exchange processes of the form $|s\rangle|p\rangle \leftrightarrow|p\rangle|s\rangle$. The coherent electronic evolution \\ of the ensemble dephases due to the variation in dipole-dipole coupling strength between \\ atom pairs in the MOT. The experimental results are in good agreement with numerical \\ calculations that simulate the interactions between nearest neighbors in a frozen gas.
}




\subsection{Introduction}

As we mentioned in Chapter 3, the large size of Rydberg atoms endows them with extreme properties which, when properly harnessed, can be exploited to study fundamental problems and applications involving the quantum control of matter in single-, few-, and many-body systems. For example, Rydberg atoms are highly-sensitivity to applied electric fields and, accordingly, to neighboring atoms, as these can induce substantial energy-shifts and/or quantum-state modification. They also exhibit long electronic time-scales, $\tau=2 \pi / E$, which characterize the evolution of superpositions of Rydberg states with small energy separations, $E$.

The strong, long-range dipole-dipole interactions that exist between neighboring Rydberg atoms couple their electronic and center-of-mass degrees of freedom [1]. Control over this coupling could enable coherent manipulation of multi-atom correlations and entanglement [2-13] with potential applications to quantum information processing [1418] or explorations of few- and many-body quantum mechanics. That said, the time- and energy-scales associated with electronic motion within individual atoms can differ substantially from those relevant to dipole-dipole couplings between atoms [1]. This disparity of scales has encouraged the segregation of work in this area, with studies of electron dynamics within atoms performed separately from those investigating interactions between atoms.

For example, in experiments exploring interactions between Rydberg atoms (e.g. resonant energy transfer [19-30], dipole blockade [2, 13, 31-36], quantum-logic gate implementation [14-18]) the interatomic coupling strengths typically range from $\mathrm{kHz}$ to tens of $\mathrm{MHz}$ with associated time-scales for the development of correlations ranging from tens of nanoseconds to milliseconds. Frequency-domain techniques and cold atomic ensembles are usually employed to enable selective excitation or high-resolution 
spectroscopy of the few- or many-body eigenstates and to limit effects associated with atom motion. Since the coupling between atoms depends on their separation, both Rydberg atom density, $\rho$ and temperature, $T$, play an important role as these determine the strength and length-scales of correlations as well as the time-scales over which coherence can be maintained.

Conversely, experiments aimed at coherently manipulating and viewing the evolution of one-electron [37-41] and two-electron [42-52] Rydberg wavepackets typically utilize time- domain methods involving ultra-fast optical and/or electric-field pulses to first excite coherent superposition states and then probe their behavior. For atoms with principal quantum number $n<100$ or so, the relevant dynamics in these experiments usually fall in the picosecond or femtosecond regime. Over these time-scales relative atom motion is completely negligible, even in thermal beams. Moreover, interactions between atoms can be ignored since their influence on the electronic evolution develops only after orders of magnitude longer times (tens of nanoseconds to milliseconds).

More generally, however, both ultrafast electron evolution and atom-atom correlations play a role in the quantum dynamics of Rydberg systems. Electron dynamics in isolated atoms set the scale for how rapidly correlations between atoms can be modified, whereas the coupling between atoms determines the minimum-time required for entanglement between pairs or groups of atoms to influence electronic evolution within them. Accordingly, potential applications may require the coherent manipulation of groups, pairs, or individual Rydberg atoms over time- and energy- scales spanning many orders of magnitude. Thus, these systems provide challenging platforms on which to explore fewand many-body quantum control.

As a step towards addressing this problem, we examine the influence of strong, resonant dipole-dipole interactions between atoms on the evolution of Rydberg wavepackets within 
those atoms. Specifically, we consider the pulsed, coherent excitation of atoms from a Rydberg eigenstate $|s\rangle$, to an adjacent level of opposite parity, $|p\rangle$, at at time $t=0$. In an extremely diffuse ensemble where the separation between atoms $R \rightarrow \infty$, the excitation would result in the creation of identical Rydberg wavepackets in each atom

$$
\Psi(t)=\cos \theta|s\rangle+e^{-i(E t+\phi)} \sin \theta|p\rangle
$$

where $E$ is the energy separation between the eigenstates, $\theta$ is an admixture coefficient, $\phi$ is an arbitrary phase related to the details of the excitation and, unless otherwise noted, atomic units are used throughout. The wavepackets are characterized by identical timedependent electric dipole-moments which oscillate with a period, $\tau=2 \pi / E$, where $E$ is the energy spacing between $s$ and $p$.

At higher densities the Rydberg electrons on each atom do not evolve independently. Each electron is affected by the multi-pole fields produced by neighboring Rydberg atoms [1]. The correlations resulting from these interactions can be non-negligible even for separations $R$ of several microns or more [30]. In the density regime explored here, $10^{8} \times$ $\mathrm{cm}^{-3}<\rho<3 \times 10^{9} \mathrm{~cm}^{-3}, R \gg r_{0}$ where $r_{0} \sim 2 n^{2}$ is the radial extent of the Rydberg wavefunction on each atom. Accordingly, resonant dipole-dipole exchange between nearest-neighbor atoms $[23,24,27,53]$ with a coupling strength on the order of $n^{4} / R^{3}(\sim 5$ MHz at $n=30$ and $\rho=10^{9} \mathrm{~cm}^{-3}$ ), dominates the atom-atom interaction. The coupling alters the eigenstates of atom pairs and, accordingly, modifies the electric-dipole oscillations within them. We measure, as a function of density, the influence of the dipoledipole exchange interaction on the coherent dipole oscillations in the Rydberg ensemble.

\subsection{Experimental Procedure}

In Chapter 2, we talked about the general experiment setup. More details about the experimental approach of this project is provided below. 
In the experiments, ${ }^{85} R b$ atoms at $\sim 70 \mu \mathrm{K}$ in a magneto-optical trap (MOT) are laserexcited from the $5 p_{3 / 2}$ upper cycling-level to the $32 s$ Rydberg state. The atoms are then exposed to a picosecond $\mathrm{THz}$ pulse which coherently redistributes a fraction $\sim 20 \%$ of the $32 s$ population to neighboring $31 p$ and $32 p$ levels which lie approximately $4.79 \mathrm{~cm}^{-1}$ and $4.35 \mathrm{~cm}^{-1}$ below and above the initial state, respectively. Excitation of other levels is negligible. The mixed-parity Rydberg wavepackets are allowed to freely evolve for a variable time $\Delta t$ before they are subjected to a second, identical $\mathrm{THz}$ pulse. The probability amplitude transfer during the second $\mathrm{THz}$ pulse interferes with that from the first, resulting in a $\Delta$ t-dependent modulation in the net population in each Rydberg state. State-selective field ionization (SSFI) is used to measure the final Rydberg eigenstate distribution as a function of $\Delta t$ and $\rho$.

The initial Rydberg excitation is performed using a $10 \mathrm{~ns}$, dye-amplified pulse from a $\sim 482 \mathrm{~nm}$ cw diode laser. A $10 \mathrm{~ns}$ pulse is switched in from the cw laser by applying a highvoltage pulse to a Pockells cell that is positioned between crossed polarizers. The two-stage dye-amplifier is pumped at $15 \mathrm{~Hz}$ by the third harmonic of a Nd:YAG laser. The $482 \mathrm{~nm}$ laser pulse is focused into the MOT using a $500 \mathrm{~mm}$ spherical lens. The freely propagating picosecond $\mathrm{THz}$ pulses are produced via optical rectification of $2 \mathrm{~mJ}, 150 \mathrm{fs}, 790 \mathrm{~nm}$ laser pulses in $\mathrm{LiNbO}_{3}$, using a tilted-pulse-front pumping scheme $[54,55]$. The $790 \mathrm{~nm}$ pulses are generated in a $15 \mathrm{~Hz}$ Ti:Sapphire regenerative amplifier. A Michelson interferometer, with a variable-length delay-stage in one arm, is used to split each $790 \mathrm{~nm}$ pulse into a pump-probe pair with a delay $0 \leqslant \Delta t<16 \mathrm{~ns}$. The two $790 \mathrm{~nm}$ pulses are collinearly incident on the $\mathrm{LiNbO}_{3}$ crystal, producing a pair of identical, co-propagating broadband single-cycle THz pulses [56]. After exiting the $\mathrm{LiNbO}_{3}$ crystal, the $\mathrm{THz}$ beam is collected by a $50 \mathrm{~mm}$ diameter, $50 \mathrm{~mm}$ focal length off-axis paraboloid. It is then weakly focused by a Teflon lens, through a thin fused silica window, into the MOT. The ramped-field that 
performs the SSFI is applied to the atoms approximately $100 \mathrm{~ns}$ after the second THz pulse. Four thin, parallel, stainless steel rods are positioned in a rectangular array surrounding the $0.5 \mathrm{~mm}$ diameter atom cloud, and enable the application of a spatially-uniform field while providing optical access for the trapping beams, Rydberg excitation laser, and THz pulses. $\mathrm{Rb}$ ions produced during the field ramp are pushed toward a micro-channel plate detector and, in principle, population in different Rydberg states can be distinguished by different ionization times in the ramped field. In practice, the signals associated with the $32 s$ and $31 p$ states appear at the same time, thus we do not separately measure the populations in these two states. The delay, $\Delta t$ between the THz pulses is scanned continuously while integrating the SSFI signals across two time bins, yielding the populations in $32 s+31 p$ states and $32 p$ level, respectively. The Rydberg atom density, $10^{8} \mathrm{~cm}^{-3}<\rho<3 \times 10^{9} \mathrm{~cm}^{-3}$, is varied by changing the current to the getters that supply the Rb atoms to the MOT. At these densities, with $T=70 \mu \mathrm{K}$, relative atom motion due to thermal energy or dipole-dipole forces is negligible during the $\sim 100 \mathrm{~ns}$ duration of the experiments.

\subsection{Experimental Results}

Figure 5.1 shows the measured population in the $32 s+31 p$ states as a function of the delay $\Delta t$ between the two $\mathrm{THz}$ pulses. The modulations in the population have a frequency of $4.35 \mathrm{~cm}^{-1}$, corresponding to the energy separation between the $32 s$ and the $32 p$ states, and reflect the oscillation of the electric dipole-moment of the wavepacket that is created by the first $\mathrm{THz}$ pulse. Oscillations at the same frequency are observed in the $32 p$ population, but are 180 degrees out of phase. The modulations can be interpreted as the result of time-domain Ramsey interference in the $32 s$ and $32 p$ amplitudes created by the first and second $\mathrm{THz}$ pulses $[57,58]$. Accordingly, the amplitude of the oscillations provides a measure of the macroscopic wavepacket coherence. Although the broadband $\mathrm{THz}$ pulses also excites atoms from $32 s$ to $31 p$ we do not observe any significant signal 
modulations at $4.79 \mathrm{~cm}^{-1}$, the frequency corresponding to the $32 s-31 p$ energy interval. For the weak population transfers studied here, any oscillations in the $32 s$ population would be out of phase with those in $31 p$, resulting in no net modulation in the combined $32 s+31 p$ signal that we detect. Moreover, the small variations in the $32 s$ amplitude associated with the $31 p$ excitation have negligible influence on the population transfer to $32 p$, so no 4.79 $\mathrm{cm}^{-1}$ beat is observed in the $32 p$ signal.
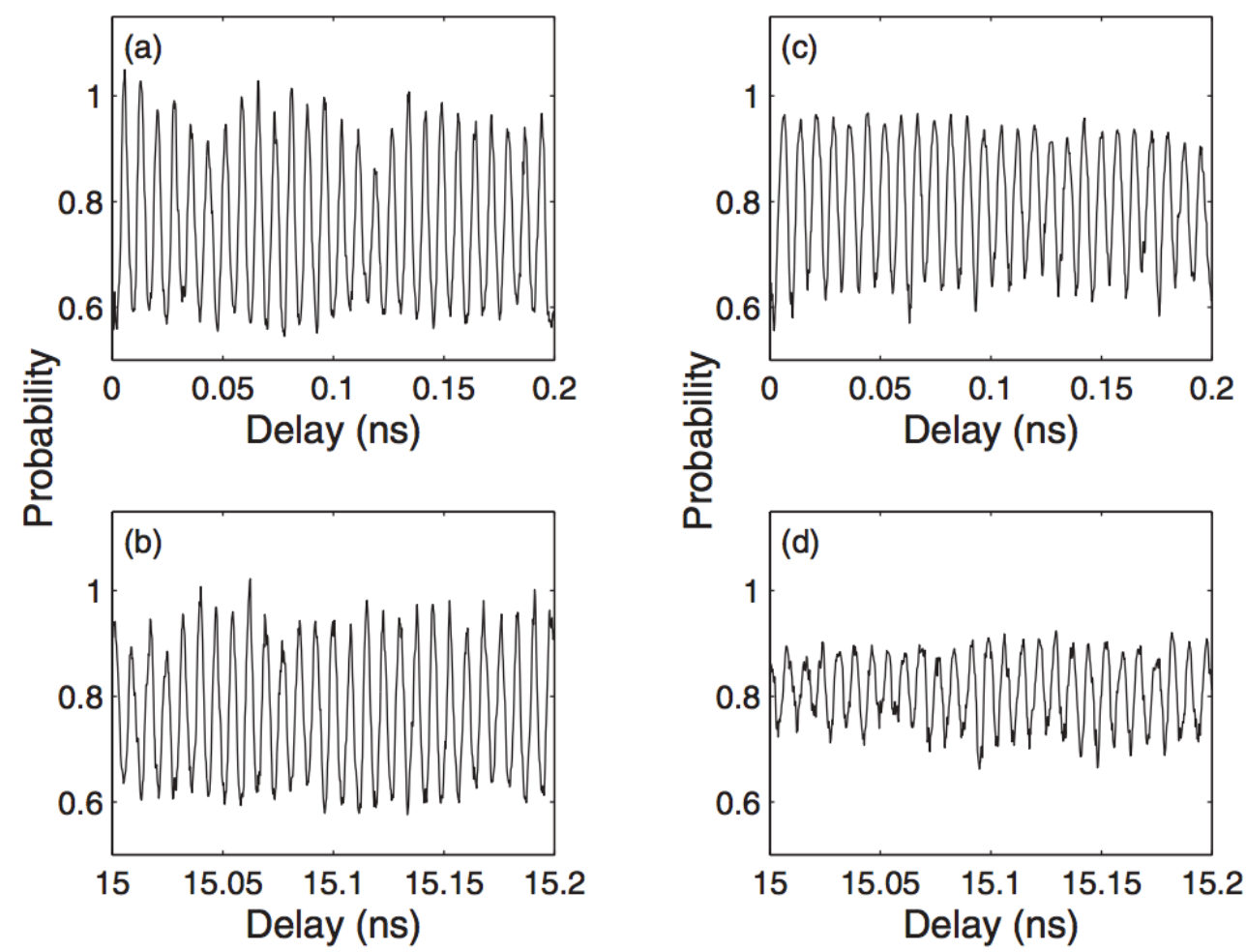

Figure 5.1: Measured population in the combined $32 s+31 p$ states as a function of the delay $\Delta t$ between two $\mathrm{THz}$ pulses. The left panels show data collected at low Rydberg density, $\rho \sim 3 \times 10^{8} \mathrm{~cm}^{-3}$, for (a) short $(\Delta t \simeq 0)$ and (b) long $(\Delta t \simeq 15 \mathrm{~ns})$ delays, respectively. The right panels show data collected at high Rydberg density, $\rho \sim 2 \times 10^{9} \mathrm{~cm}^{-3}$, for c) short $(\Delta t \simeq 0)$ and d) long ( $\left.\Delta t \simeq 15 \mathrm{~ns}\right)$ delays, respectively. The decrease in oscillation amplitude at high density and long delays is apparent.

Figure 5.1a and 5.1b show the situation at low density $\rho \sim 3 \times 10^{8} \mathrm{~cm}^{-3}$ for short $(\Delta t \simeq$ 0 ) and long ( $\Delta t \simeq 15 \mathrm{~ns})$ delays, respectively. Analogous plots at higher density $\rho \sim 2 \times 10^{9}$ $\mathrm{cm}^{-3}$ are shown in Figure 5.1c and 5.1d. At low density, the average oscillation amplitudes are similar at short and long delays. However, at higher densities, there is a notable decrease 
in oscillation amplitude with increasing delay. This density-dependent decay in the macroscopic wavepacket coherence, i.e. dephasing, is the principal focus of this chapter.

To quantify the average oscillation amplitude during a given time interval, we Fourier transform the delay-dependent data, and compute the area under the spectral feature (between $4.0 \mathrm{~cm}^{-1}$ and $4.6 \mathrm{~cm}^{-1}$ ) corresponding to the $32 s-32 p$ quantum beat. Figure 5.2 shows the Fourier transforms of the data in Figure 5.1. For each Rydberg density, we compute a decay factor, $\eta$, defined as the ratio of the spectral area measured near delay $\Delta t$ $=15 \mathrm{~ns}$ to that measured near $\Delta t=0$.
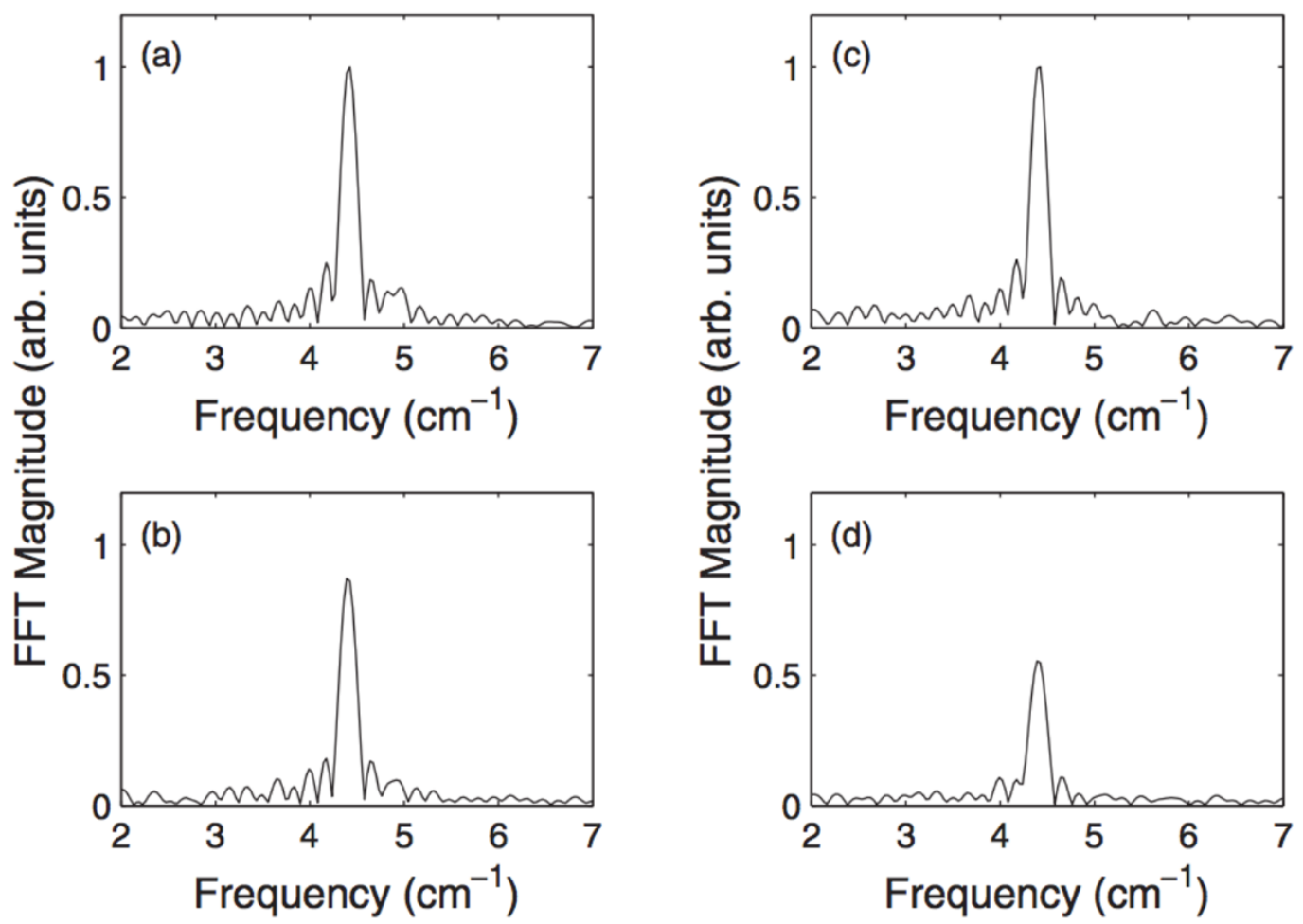

Figure 5.2: Fast Fourier transform (FFT) of the delay-dependent populations shown in Figure 5.1.

The experimentally determined values of $\eta$ are plotted vs Rydberg density in Figure 5.3 along with the results of a quantum simulation that considers the dipole-dipole interaction between nearest neighbor atoms in a frozen ensemble of randomly distributed Rydberg atoms. The simulation is in good agreement with the measurements. Before discussing the 
details of the full simulation, we present a simple model that captures the essential physics. Namely, the decrease in $\eta$ with increasing density is due to the variation in the strength of the dipole-dipole exchange interaction for different pairs of nearest neighbor atoms.

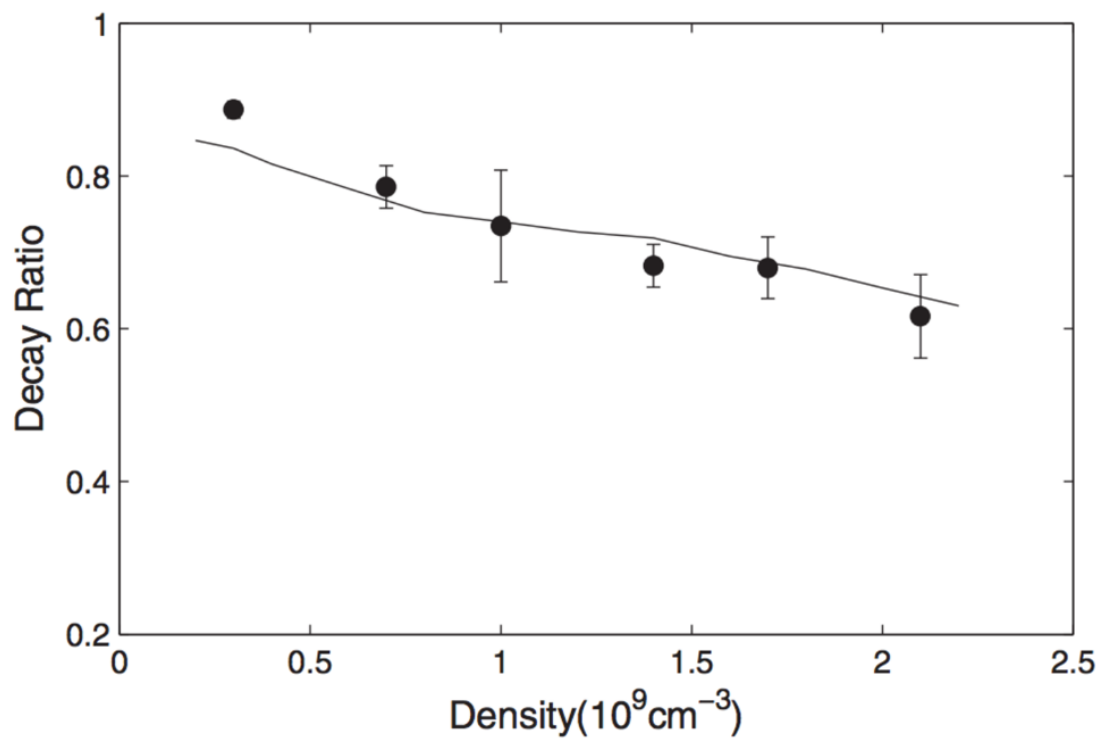

Figure 5.3: Measured (filled circles) and simulated (solid curve) decay ratio, $\eta$, as a function of Rydberg density.

\subsection{Discussion}

Consider a two-level Rydberg atom with opposite parity, non-degenerate eigenstates, $|s\rangle$ and $|p\rangle$, that are split by an energy $E_{0}$. Ignoring the azimuthal degrees of freedom, the eigenstates for a pair of atoms with a large separation $R \rightarrow \infty$ are: $|s s\rangle,|s p\rangle,|p s\rangle$, and $|p p\rangle$, with energies as shown on the left in Figure 5.4. For smaller values of $R$, the pair eigenstates are modified due to the interactions between the atoms. Provided that $R$ remains sufficiently large that the Rydberg wavefunctions of the individual atoms do not overlap, the predominant interaction between the atoms is given by the dipole-dipole coupling [53],

$$
V=\left[\overrightarrow{\mu_{A}} \cdot \overrightarrow{\mu_{B}}-3\left(\overrightarrow{\mu_{A}} \cdot \hat{R}\right)\left(\overrightarrow{\mu_{B}} \cdot \hat{R}\right)\right] / R^{3}
$$


as we mentioned in Chapter 4. The matrix elements coupling $|s s\rangle$ or $|p p\rangle$ to $|s p\rangle$ and $|p s\rangle$ are identically zero. In addition, provided $2 E_{0}$ is much greater than the magnitude of the matrix element connecting $|s s\rangle$ and $|p p\rangle$, the Hamiltonian is approximately diagonalized by considering only the coupling between the degenerate states $|s p\rangle$, $|p s\rangle$, i.e. the dipole-dipole exchange interaction. The modified energy levels are shown on the right in Figure 5.4, with eigenstates $|+\rangle$ and $|-\rangle$ corresponding to symmetric and antisymmetric combinations of $|s p\rangle$, $|p s\rangle$. The exchange splitting between this pair of entangled states is $2 \varepsilon=2\langle p s|V| s p\rangle$ which implicitly depends on the atom separation, $R$.

A THz pulse with a central frequency $E_{0}$ and a bandwidth $\gg \varepsilon$ can excite the atom pair from $|s s\rangle$ to $|+\rangle$, via a one-photon excitation, or to $|p p\rangle$ through two-photon absorption. However, $|-\rangle$ is not populated since the transition matrix elements connecting it to all other levels are identically zero. For a relatively weak $\mathrm{THz}$ pulse, the $|p p\rangle$ excitation probability is negligible, so an electronic coherence is established between $|s s\rangle$ and $|+\rangle$, and the electronic wavefunction has the form, $\Psi(t)=\cos \theta|s s\rangle+e^{-i(E t+\phi)} \sin \theta|+\rangle$, where $\theta$ depends on the excitation probability and $\phi$ is an excitation phase. This wavepacket has the same form as the single electron wavepacket in Equation (5.1) and, similarly, is characterized by a time-dependent dipole moment which oscillates sinusoidally at a frequency, $E=E_{0}+\varepsilon$. When the atoms are exposed to a second $\mathrm{THz}$ pulse, the net population transfer from $|s s\rangle$ to $|+\rangle$ is delay-dependent, oscillating at a frequency $E$. This modulation can be observed in the total population of individual atoms in states $|s\rangle$ or $|p\rangle$. It can be viewed as the result of time-domain Ramsey interference in the probability amplitude transferred from $|s s\rangle$ to $|+\rangle$ in each of the two THz pulses [57, 58]. Alternatively, but equivalently, it can be attributed to the variations in the wavepacket's instantaneous electric dipole moment during the second $\mathrm{THz}$ pulse. 


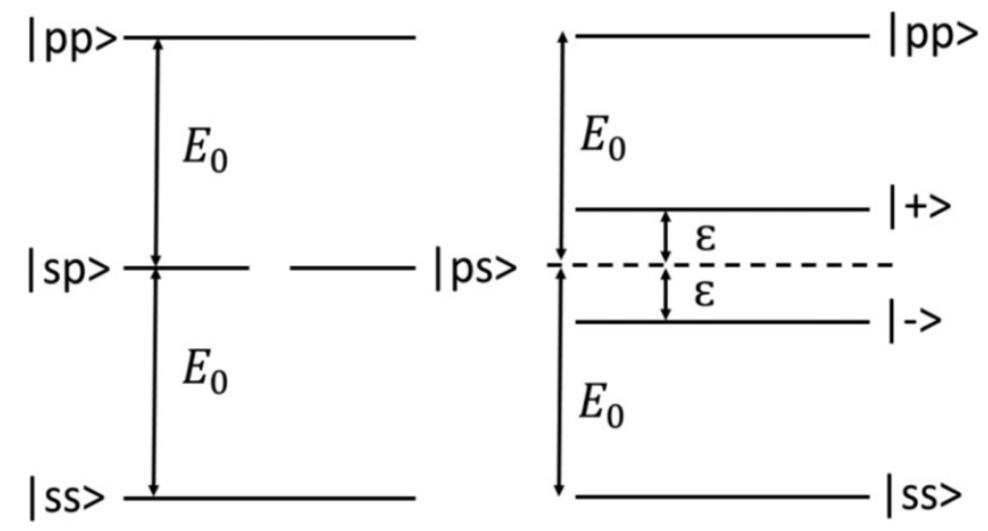

Figure 5.4: Schematic energy level diagram for the eigenstates of a pair of two level atoms. The diagrams on the left and right sides of the figure depict the situation at large and small interatomic spacing, $R$, respectively.

In a random ensemble of atoms, the dominant coupling is between each atom and its nearest neighbor. Due to the variation in $R$ for different atom pairs, there is a broad distribution of dipole-dipole exchange energies $2 \varepsilon$ and, accordingly, a range of wavepacket oscillation frequencies $E$ across the sample. This inhomogeneity results in a dephasing of the detected oscillations in the $|s\rangle$ and $|p\rangle$ populations. The dephasing time decreases with increasing Rydberg density, since the range of possible exchange energies grows with the probability of finding atom pairs with smaller $R$. It is important to note, however, that the macroscopic dephasing is not an indicator of microscopic decoherence of individual atom pairs.

In the experiments, limitations in the maximum path length difference in the arms of the Michelson interferometer preclude our measurement of the wavepacket evolution from its initiation through complete dephasing. Instead, we use $\eta$ as a measure of the dephasing rate. Since smaller values of $\eta$ reflect more rapid dephasing, the data in Figure 5.3 confirm the qualitative prediction of the model presented in the preceding paragraphs.

To determine if nearest neighbor interactions are sufficient to cause the densitydependent dephasing that is observed, we perform quantum simulations to obtain a 
quantitative prediction of the dephasing rate. Specifically, we numerically integrate the time-dependent Schroedinger equation to calculate the delay-dependent probability for finding atoms in a range of essential Rydberg states following the exposure of a random ensemble of $32 s$ atoms to two single-cycle $\mathrm{THz}$ pulses [56] like those used in the experiments. We include $33 s, 32 p, 32 s$, and $31 p$ states, with fine-structure, and consider the excitation to, and from, Rydberg-Rydberg pairs with all allowable values of $M$, the quantum number corresponding to the projection of total angular momentum along $\vec{R}$. Atom pairs with different $M$ possess different interaction energies, even for the same value of $R$, providing an additional source of inhomogeneity in the oscillation frequency of different wavepackets in the ensemble [29, 59]. Radial matrix elements are computed using a Numerov algorithm [60] with the known quantum defects of the Rb $n s$ and $n p$ states. The simulation results for individual atom pairs are integrated over the nearest neighbor distribution function for $R$ for a given Rydberg density [61]. Since the quantization axis for each atom pair is chosen to lie along $\vec{R}$, the THz polarization angle relative to that axis is varies from one atom pair to the next (refer to 3.3.4). We find that the simulation results are insensitive to whether we explicitly perform the calculation over all polarization angles and average those results, or if we fix the polarization angle at a value for which the interaction potential is equal to angle-averaged value. Since the latter method substantially improves the calculation speed, we use it for the results shown in Figure 5.3.

Inspection of Figure 5.3 shows that the agreement between the data and simulation is good. The fact that there are no adjustable parameters in the calculation, indicates that nearest neighbor interactions are sufficient to explain the observed dephasing. Beyond the limited pump-probe delay range available to the experiment, the simulations show that the degree and rate of dephasing continue to increase at longer delays and at higher densities, respectively. 


\subsection{Conclusion}

We have explored the evolution of Rydberg wavepackets in the presence of strong dipole-dipole interactions in a frozen gas. The time-scales associated with oscillation of the Rydberg electric dipole moment ( $\sim 8 \mathrm{ps})$ and the interatomic excitation exchange ( $\sim 200 \mathrm{~ns})$ differ by over four orders of magnitude yet we are able to probe the system for a sufficiently long time to observe the influence of the atom-atom coupling. The distribution of atom separations results in an inhomogeneity in the strength of the exchange coupling between neighboring atoms, causing a dephasing of the macroscopic coherence in tens of ns. In analogy to recent work with cold polar molecules [62], future experiments may take advantage of optical confinement of atoms at well-defined separations to explore the use of the dipole-dipole coupling as a controllable tool for manipulating multi-electron correlation and dynamics. 


\section{Bibliography}

[1] Thomas F. Gallagher. Rydberg Atoms. Cambridge University Press (1994).

[2] M. D. Lukin, M. Fleischhauer, R. Cote, L.M. Duan, D. Jaksch, J.I. Cirac, and P. Zoller, Phys. Rev. Lett. 87, 037901 (2001).

[3] T. Wilk, A. Gaetan, C. Evellin, J. Wolters, Y. Miroshnychenko, P. Grangier, and A. Browaeys, Phys. Rev. Lett. 104, 010502 (2010).

[4] Hendrik Weimer, Robert Low, Tilman Pfau, and Hans Peter Buchler, Phys. Rev. Lett. $101,250601(2008)$.

[5] T. Pohl, E. Demler, and M. D. Lukin, Phys. Rev. Lett. 104, 043002 (2010).

[6] S. Wuster, C. Ates, A. Eisfeld, and J. M. Rost, Phys. Rev. Lett. 105, 053004 (2010).

[7] Jens Honer, Hendrik Weimer, Tilman Pfau, and Hans Peter Buchler, Phys. Rev. Lett. $105,160404(2010)$.

[8] A. Schwarzkopf, R. E. Sapiro, and G. Raithel, Phys. Rev. Lett. 107, 103001 (2011).

[9] Matthieu Viteau, Paul Huillery, Mark G. Bason, Nicola Malossi, Donatella Ciampini, Oliver Morsch, Ennio Arimondo, Daniel Comparat, and Pierre Pillet, Phys. Rev. Lett. 109, $053002(2012)$.

[10] D.D.Bhaktavatsala Rao and K. Molmer, Phys. Rev. Lett. 111, 033606 (2013).

[11] M. Ebert, A. Gill, M. Gibbons, X. Zhang, M. Saffman, and T.G. Walker, Phys. Rev. Lett. 112, 043602 (2014).

[12] H. Schempp, G. Gunter, M. Robert-de-Saint-Vincent, C.S. Hofmann, D. Breyel, A. Komnik, D.W. Schonleber, M. Garttner, J. Evers, S. Whitlock, and M. Weidemuller, Phys. Rev. Lett. 112013002 (2014).

[13] D. Barredo, S. Ravets, H. Labuhn, L. Beguin, A. Vernier, F. Nogrette, T. Lahaye, and A. Browaeys, Phys. Rev. Lett. 112, 183002 (2014). 
[14] D. Jaksch, J.I. Cirac, P. Zoller, S.L. Rolston, R. Cote, and M.D. Lukin, Phys. Rev. Lett. 85, 2208 (2000).

[15] M. Muller, I. Lesanovsky, H. Weimer, H. P. Buchler, and P. Zoller, Phys. Rev. Lett. 102, $170502(2009)$.

[16] M. Saffman, T.G. Walker, and K. Molmer, Rev. Mod. Phys. 82, 2313 (2010), and references therein.

[17] L. Isenhower, E. Urban, X. L. Zhang, A. T. Gill, T. Henage, T. A. Johnson, T. G. Walker, and M. Saffman, Phys. Rev. Lett. 104, 010503 (2010).

[18] D. Paredes-Barato and C.S. Adams, Phys. Rev. Lett. 112, 040501 (2014).

[19] K.A. Safinya, J. F. Delpech, F. Gounand, W. Sandner, and T.F. Gallagher, Phys. Rev. Lett. 47, 405 (1981).

[20] P. Pillet, R. Kachru, N. H. Tran, W. W. Smith, and T. F. Gallagher, Phys. Rev. Lett. $50,1763(1983)$.

[21] D.S. Thomson, M.J. Renn, and T.F. Gallagher, Phys. Rev. Lett. 65, 3273 (1990).

[22] M.J. Renn and T.F. Gallagher, Phys. Rev. Lett. 67, 2287 (1991).

[23] W.R. Anderson, J.R. Veale, and T.F. Gallagher, Phys. Rev. Lett. 80, 249 (1998).

[24] I. Mourachko, D. Comparat, F. de Tomasi, A. Fioretti, P. Nosbaum, V. M. Akulin, and P. Pillet, Phys. Rev. Lett. 80, 253 (1998).

[25] T. J. Carroll, K. Claringbould, A. Goodsell, M. J. Lim, and M. W. Noel, Phys. Rev. Lett. 93, 153001 (2004).

[26] A. Walz-Flannigan, J. R. Guest, J.-H. Choi, and G. Raithel, Phys. Rev. A 69, 063405(2004).

[27] S. Westermann, T. Amthor, A.L. de Oliveira, J. Deiglmayr , M. Reetz-Lamour, and M. Weidemuller, Eur. Phys. J. D 40, 37 (2006).

[28] P. Bohlouli-Zanjani, J. A. Petrus, and J. D. D. Martin, Phys. Rev. Lett. 98, 203005 (2007). 
[29] M.R. Kutteruf and R.R. Jones, Phys. Rev. A 82, 063409 (2010).

[30] M.R. Kutteruf and R.R. Jones, Phys. Rev. Lett. 108, 013001 (2012).

[31] D. Tong, S.M. Farooqi, J. Stanojevic, S. Krishnan, Y.P. Zhang, R. Cote, E.E. Eyler, and P.L. Gould, Phys. Rev. Lett. 93, 063001 (2004).

[32] K. Singer, M. Reetz-Lamour, T. Amthor, L.G. Marcassa, and M. Weidemuller, Phys. Rev. Lett. 93, 163001 (2004).

[33] T. Cubel Liebisch, A. Reinhard, P. R. Berman, and G. Raithel, Phys. Rev. Lett. 95, $253002(2005)$.

[34] T. Vogt, M. Viteau, J. Zhao, A. Chotia, D. Comparat, and P. Pillet, Phys. Rev. Lett. 97, 083003 (2006).

[35] E. Urban, T.A. Johnson, T. Henage, L. Isenhower, D.D. Yavuz, T.G. Walker and M. Saffman, Nature Physics 5, 110 (2009).

[36] A. Gatan, Y. Miroshnychenko, T. Wilk, A. Chotia, M. Viteau, D. Comparat, P. Pillet, A. Browaeys and P. Grangier, Nature Physics 5, 115 (2009).

[37] R.R. Jones and L.D. Noordam, Electronic Wavepackets, Adv. in At. Mol. Opt. Phys. 38, 1 (1997), and references therein.

[38] F.B. Dunning, J.J. Mestayer, C.O. Reinhold, S. Yoshida, and J. Burgdorfer, J. Phy. B: At. Mol. Opt. Phys. 42, 022001 (2009), and references therein.

[39] J. Bromage and C.R. Stroud, Jr., Phys. Rev. Lett. 83, 4963 (1999).

[40] H. Maeda, D.V.L. Norum, and T.F. Gallagher, Science 307, 1757 (2005).

[41] R.S. Minns, M.R. Kutteruf, H. Zaidi, L. Ko and R.R. Jones, Phys. Rev. Lett. 97, 040504 (2006).

[42] J.G. Story, D.I. Duncan, and T.F. Gallagher, Phys. Rev. Lett. 71, 3431 (1993).

[43] D. W. Schumacher, B. J. Lyons, and T. F. Gallagher, Phys. Rev. Lett. 78, 4359 (1997).

[44] Xin Chen and John A. Yeazell, Phys. Rev. Lett. 81, 5772 (1998).

[45] R. van Leeuwen, M.L. Bajema, and R.R. Jones, Phys. Rev. Lett. 82, 2852 (1999). 
[46] J.B.M. Warntjes, C. Wesdorp, F. Robicheaux, and L.D. Noordam, Phys. Rev. Lett. 83, $512(1999)$.

[47] J.E. Thoma and R.R. Jones, Phys. Rev. Lett. 83, 516 (1999).

[48] J.G. Story and H.N. Ereifej, Phys. Rev. Lett. 86, 612 (2001).

[49] R. van Leeuwen, K. Vijayalakshmi, and R.R. Jones, Phys. Rev. A 63, 033403 (2001).

[50] S.N. Pisharody and R.R. Jones, Phys. Rev. Lett. 91, 203002 (2003).

[51] S.N. Pisharody and R.R. Jones, Science 303, 813 (2004).

[52] X. Zhang, R.R. Jones, and F. Robicheaux, Phys. Rev. Lett. 110, 023002 (2013).

[53] F. Robicheaux, J. V. Hernandez, T. Topcu, and L. D. Noordam, Phys. Rev. A 70 042703 (2004).

[54] J. Hebling, Ka-Lo Yeh, M.C. Hoffmann, B. Bartal, and K. A. Nelson, JOSA B, 25, B6 (2008).

[55] H. Hirori, A. Doi, F. Blanchard, and K. Tanaka, Appl. Phys. Lett. 98, 091106 (2011).

[56] S. Li and R.R. Jones, Phys. Rev. Lett. 112, 143006 (2014).

[57] L.D. Noordam, D.I. Duncan, and T.F. Gallagher, Phys. Rev. A 45, 4734 (1992).

[58] N.E. Tielking and R.R. Jones, Phys. Rev. A 52, 1371 (1995).

[59] H. Park and T.F. Gallagher, Phys. Rev. A 86, 052510 (2012).

[60] M.L. Zimmerman, M.G. Littman, M.M. Kash, and D. Kleppner, Phys. Rev. A 20 $2251(1979)$

[61] P. Hertz, Math. Ann. 67387 (1909).

[62] B. Yan, S.A. Moses, B. Gadway, J.P. Covey, K.R.A. Hazzard, A.M. Rey, D.S. Jin, and J. Ye, Nature 501, 521 (2013). 


\title{
6 Wavepacket Coherence Transfer via DD interactions
}

\begin{abstract}
We have shown that electron correlations, induced by controlled DD interactions, can enable the coherent transfer of electronic wavepacket motion from atoms to their neighbors. In the experiment, a $5 \mathrm{~ns}$ tunable dye laser excites $\mathrm{Rb}$ atoms in a MOT to the $25 \mathrm{~s}$ state in a weak static electric field for which the tunable $25 s 33 s \leftrightarrow 24 p 34 p$ DD interaction is resonant. A picosecond $\mathrm{THz}$ pulse then further excites each Rydberg atom into a coherent superposition, of $25 \mathrm{~s}$ and $24 \mathrm{p}$ states. The evolution of this mixed-parity wavepacket is characterized by time-dependent oscillations in the electric dipole moment, with a period of 2.9 ps. Approximately $5 \mathrm{~ns}$ after the wavepacket creation, a second $5 \mathrm{~ns}$ dye-laser promotes a second set of atoms from the $5 p$ level into the $33 s$ state. Because of the DD interaction, the second dye laser actually creates atom pairs whose electronic states are correlated via the resonant DD coupling. A $33 s+34 p$ wavepacket, oscillating with the same $2.9 \mathrm{ps}$ period as the $25 s+24 p$ wavepacket, develops on the second set of atoms as a result of the correlation. A second, time-delayed ps $\mathrm{THz}$ pulse enables the detection of the coherent wavepacket motion on the two sets of atoms.
\end{abstract}




\subsection{Introduction}

Quantum control aims to steer a quantum system from an initial state to a target state via an external field. Because of its potential scientific benefits and practical applications, ranging from chemical reactions [1][2] to nuclear magnetic resonance (NMR) [3], and from laser cooling [4][5] to quantum information processing [6][7], it has attracted considerable interest in recent years. In this chapter, we describe an experiment which utilizes coherent manipulation of DD interactions to exert quantum control over correlated electronic wavepacket motion on neighboring atoms in a frozen gas.

In radio and telecommunications, an oscillating current (usually a semi-sinusoidal wave) drives the two poles of an antenna, creating a transmitter [8]. The transmitter broadcasts an electromagnetic wave which, in turns drives the two poles of a properly tuned receiver antenna. This process can be viewed as a macroscopic classical physics example of resonant coherence and energy transfer. At the microscopic scale, DD interactions can enable analogous resonant transfer via near-field, rather than far field, electromagnetic coupling. For example, in photosynthetic systems, light-harvesting antennas transmit light energy to reaction centers via DD interactions [9]. There is currently significant debate as to whether quantum coherence is maintained in this process.

We have explored a similar situation in a system where quantum coherence should be maintained. Consider a Rydberg atom in a coherent superposition of $s$ and $p$ states: $|\varphi\rangle=$ $\left(|s\rangle+|p\rangle e^{-i \Delta E t}\right) / \sqrt{2}$, where $\Delta E$ is the energy gap between the $s$ and $p$ states. The timedependent dipole moment of this atom is $\langle\varphi|\vec{z}| \varphi\rangle=2 \cos (\Delta E t)\langle s|\vec{z}| p\rangle$. As the electronic wavepacket evolves, the dipole moment of this atom is changing periodically or "oscillating", in analogy to a classical dipole transmitter. We consider the case where there is a microscopic "receiver" (another Rydberg atom) in the vicinity such that the wavepacket 
motion on one atom can induce a wavepacket with a coherently oscillating dipole moment on the other.

Consider an atom pair $\mathrm{AB}$ initially in state $\varphi(0)=|s\rangle\left|s^{\prime}\right\rangle$ in a system where resonant energy transfer can occur to a second pair state $|s\rangle\left|s^{\prime}\right\rangle \leftrightarrow|p\rangle\left|p^{\prime}\right\rangle$. At resonance, the wavefunction of this atom pair can be written as:

$$
\varphi(t)=\frac{1}{\sqrt{2}}\left(|+\rangle e^{-i \varepsilon t}+|-\rangle e^{i \varepsilon t}\right)
$$

where $|+\rangle=\frac{1}{2}\left(|s\rangle\left|s^{\prime}\right\rangle+|p\rangle\left|p^{\prime}\right\rangle\right),|-\rangle=\frac{1}{2}\left(|s\rangle\left|s^{\prime}\right\rangle-|p\rangle\left|p^{\prime}\right\rangle\right)$ and $\varepsilon$ is the DD interaction strength (see Figure 6.1). Equation (6.1) can also be written as:

$$
\varphi(t)=\cos \varepsilon t|s\rangle\left|s^{\prime}\right\rangle-i \sin \varepsilon t|p\rangle\left|p^{\prime}\right\rangle
$$

When $t=\frac{N \pi}{\varepsilon}$, Equation (6.2) shows that the pair has returned to the initial $|s\rangle\left|s^{\prime}\right\rangle$ state. When $t=\frac{(2 N+1) \pi}{2 \varepsilon}$, the pair has been transferred with unit probability to $|p\rangle\left|p^{\prime}\right\rangle$. In general, there is a non-zero probability of finding atoms A and B in states $|s\rangle$ and $|p\rangle$ and $\left|s^{\prime}\right\rangle$ and $\left|p^{\prime}\right\rangle$, respectively. However, at no time is there a coherent wavepacket with well defined phases on the individual atoms. Instead, $\varphi(t)$ is an entangled state and there is no separable wavepacket on either atom A or B. If we measure the dipole moment on either A or B separately, we obtain zero.

To enable the observation of wavepacket coherence transfer from one atom to another, we consider the case where one atom in the pair is initially prepared in a coherent superposition state and the other is in an eigenstate. In this pair, atom A starts from a wavepacket composed of states $|s\rangle$ and $|p\rangle$, and acts as the "transmitter", while atom B starts from an eigen state $\left|s^{\prime}\right\rangle$ and acts as the "receiver". We further assume that there is a resonant coupling between the pair states, $|s\rangle\left|s^{\prime}\right\rangle \leftrightarrow|p\rangle\left|p^{\prime}\right\rangle$.

Suppose, initially at $t=0$, the wavefunction of this pair is: 


$$
\varphi(t=0)=\frac{1}{\sqrt{2}}(|s\rangle+|p\rangle)\left|s^{\prime}\right\rangle
$$

Due to the DD coupling, after a time T, the wavefunction can be written in a coupled atom basis as:

$$
\varphi(T)=\frac{1}{2}\left(|+\rangle e^{-i \varepsilon T}+|-\rangle e^{i \varepsilon T}\right)+\frac{1}{\sqrt{2}}|p\rangle\left|s^{\prime}\right\rangle e^{i \Delta E T} .
$$

where $\Delta E$ is the energy gap between $|s\rangle$ and $|p\rangle$, as well as between $|s\rangle\left|s^{\prime}\right\rangle$ and $|p\rangle\left|s^{\prime}\right\rangle$ (see Figure 6.1). Rewriting equation (6.4) in the independent atom basis:

$$
\begin{aligned}
\varphi(T)= & \frac{1}{2 \sqrt{2}}\left(\left(|s\rangle\left|s^{\prime}\right\rangle+|p\rangle\left|p^{\prime}\right\rangle\right) e^{-i \varepsilon T}+\left(|s\rangle\left|s^{\prime}\right\rangle-|p\rangle\left|p^{\prime}\right\rangle\right) e^{i \varepsilon T}\right) \\
& +\frac{1}{\sqrt{2}}|p\rangle\left|s^{\prime}\right\rangle e^{i \Delta E T}
\end{aligned}
$$

Assuming the DD coupling is switched off for $t>T$, we can write

$$
\varphi(t)=\left(\cos \varepsilon T|s\rangle\left|s^{\prime}\right\rangle-i \sin \varepsilon T|p\rangle\left|p^{\prime}\right\rangle+|p\rangle\left|s^{\prime}\right\rangle e^{i \Delta E t}\right) / \sqrt{2}
$$

or

$$
\begin{aligned}
\varphi(t) & =\frac{1}{\sqrt{2}} \cos \varepsilon T\left(|s\rangle+\cos \varepsilon T|p\rangle e^{i \Delta E t}\right)\left|s^{\prime}\right\rangle \\
& -\frac{i}{\sqrt{2}} \sin \varepsilon T|p\rangle\left(\left|p^{\prime}\right\rangle+i \sin \varepsilon T\left|s^{\prime}\right\rangle e^{i \Delta E t}\right)
\end{aligned}
$$

Equation (6.7) describes an entangled state where the wavepacket is on either atom, but on one, and only one, of the two atoms. When $T=\frac{N \pi}{\varepsilon}$, atom A is in a superposition state and B is in an eigenstate. When $T=\frac{(2 N+1) \pi}{2 \varepsilon}$, due to the DD coupling, atom A is left in an eigenstate while a coherent superposition state has developed on atom B. So the DD interaction coherently splits the atom pair into two distinct pieces, with atom A (or B) in an eigenstate and atom B (or A) in a coherent superposition (see Figure 6.2 and Figure 6.3). The two wavepackets exhibit time-dependent dipole moments oscillating with the same period and well-defined, but different phases. Because the wavepackets on both atoms have well defined phases, we should be able to detect the wavepackets on both of them using the optical Ramsey method [10], as described in Chapter 5. 


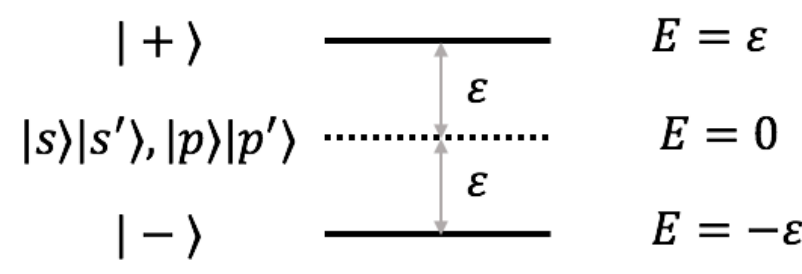

$|p\rangle\left|s^{\prime}\right\rangle \quad \longrightarrow \quad E=-\Delta E$

Figure 6.1: Schematic energy level diagram of an atom pair. As described in previous chapters, DD interactions break the energy degeneracy and create an energy gap between the coupled pair states.
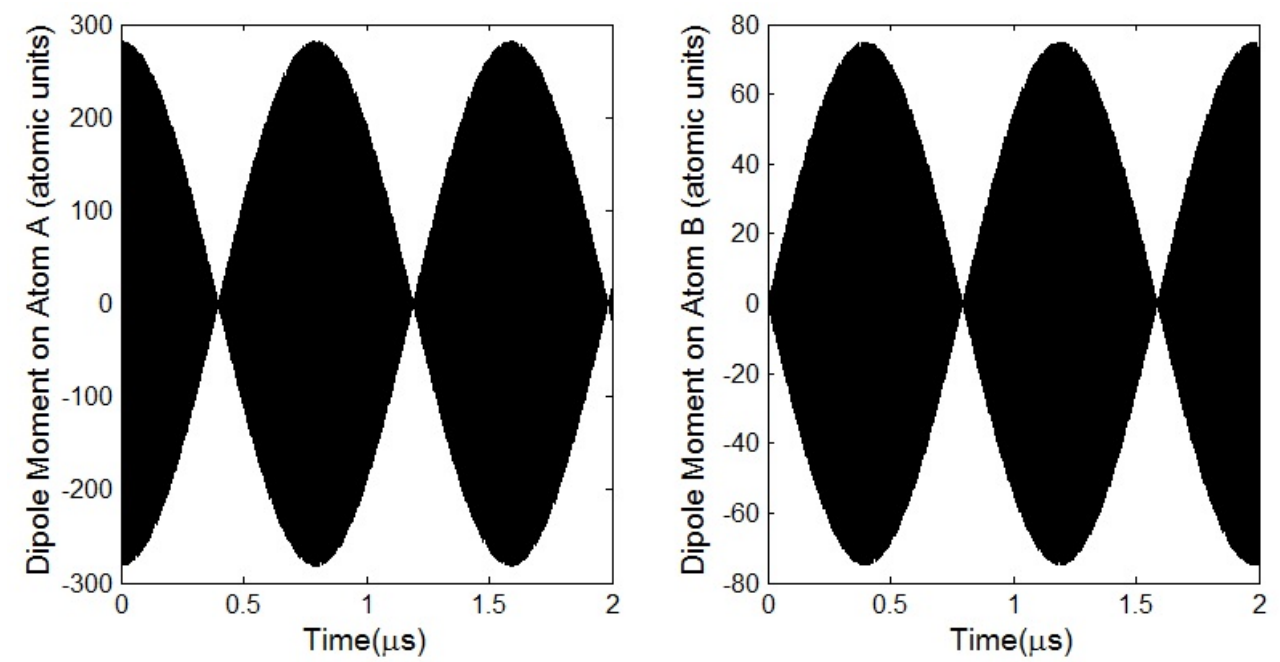

Figure 6.2: Calculated time dependent dipole moment along the $z$ axis for each atom in an atom pair in the presence of a resonant energy coupling $25 s 33 s \leftrightarrow 24 p_{1 / 2} 34 p_{3 / 2}$. Atom A is initially in a coherent superposition of $25 s+24 p$ (left figure) and atom B is initially in an eigenstate $33 \mathrm{~s}$ (right figure). The dipole moment on each atom has a fast oscillation (not resolved in the figure) with a period of $\sim 2.9$ ps modulated by a slow oscillation with a period of $\sim 0.8 \mu \mathrm{s}$, associated with the strength of the DD interaction. The distance between the two atoms is set to be $\sim 4 \mu \mathrm{m}$ the most probable nearest-neighbor separation in an ensemble at a density of $\sim 2 \times 10^{9} \mathrm{~cm}^{-3}$. The magnitude of the DD interaction is $\sim 7 \mathrm{MHz}$. The figures show that the wavepacket coherence travels back and forth between the atoms in one pair. 

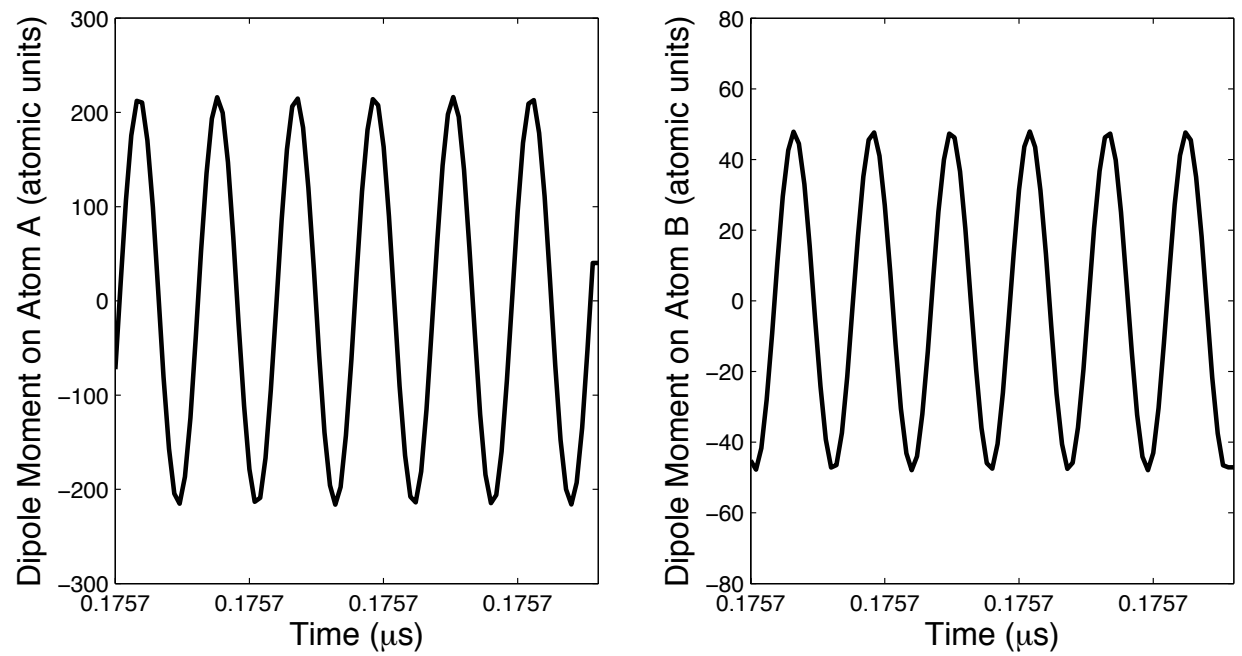

Figure 6.3: Magnified view of the dipole oscillation shown in Figure 6.2. Note the identical period of, but $90^{\circ}$ phase difference between, the oscillations on the two atoms.

For a single pair of frozen atoms, the DD interaction strength is fixed, the coherence of the correlated wavepacket is maintained, and the dipole motion continues to oscillate back and forth between the two atoms. There is no dephasing as the dipole oscillation and wavepacket transfer frequencies are constant and well-defined. However, for a random ensemble of atoms, the wavepacket motion should dephase due to the variation in the DD coupling strength for different atom pairs in the MOT. For a random ensemble containing $\sim 10^{4}$ atoms, it is a big challenge to maintain macroscopic wavepacket coherence over the ensemble [11]. Specifically, it is necessary to detect the wavepacket transfer before the dephasing is complete.

\subsection{Experimental Procedure}

We use a system that has been explored in detail [12][13] to demonstrate wavepacket transfer between atoms via electron correlation. Specifically, we consider the resonant reaction: $25 s 33 s \leftrightarrow 24 p_{1 / 2} 34 p_{3 / 2}$. To relate to the theory discussed above, we define $s=25 \mathrm{~s}$, $s^{\prime}=33 \mathrm{~s}, p=24 p_{1 / 2}$ and $p^{\prime}=34 p_{3 / 2}$. For simplicity, $34 p$ is used to represent $34 p_{3 / 2}$ and $24 p$ to represent $24 p_{1 / 2}$ in the following discussion, unless stated otherwise. When a static offset field of about $3 \mathrm{~V} / \mathrm{cm}$ is applied, the energy gap between $25 s$ and $24 p_{1 / 2}$ is $\sim 11.4$ 
$\mathrm{cm}^{-1}$, which is the same as the gap between $33 s$ and $34 p$, so that resonant coupling between the atom pairs can occur.

In the experiment, cold $\mathrm{Rb}$ atoms are trapped in a MOT. A nanosecond dye laser pulse saturates the transition from the upper trap state, $5 p_{3 / 2}$, to $25 s$. These Rydberg atoms, considered as atoms $\mathrm{A}$, are then exposed to a picosecond pulse of $\mathrm{THz}$ radiation to create a coherent superposition state composed of a roughly equal admixture of $25 s$ and $24 p$. Immediately after the $\mathrm{THz}$ pulse, a second nanosecond dye laser pulse promotes another portion of the $5 p_{3 / 2}$ amplitude in the trapped atoms to $33 s$. Technically, in each laser shot, all atoms are excited to both a $25 s+24 p$ wavepacket and to the $33 \mathrm{~s}$ state with some probability. However, since there is no phase coherence between the dye laser pulses which excite $25 s$ and $33 s$, respectively, in practice the problem is identical to one in which some atoms are excited to $25 s+24 p$ and others are excited to $33 s$. The $33 s$ atoms are considered to be atoms B. Our measurements are most sensitive to nearest neighbor atom pairs $(|25 s\rangle$ $+|24 p\rangle)|33 s\rangle$ for which resonant coupling can produce atoms in the $34 p$ states.

After the second dye laser excitation, the atoms are allowed to interact resonantly for a period $T$ until they are ionized by a ramped field. If a coherent superposition of $33 s$ and $34 p$ is formed in atoms $\mathrm{B}$, the wavepacket motion can be probed by applying a second picosecond $\mathrm{THz}$ pulse at the end of the interaction time $T$, just before the field ionization ramp, as described below (refer to Figure 6.4). 


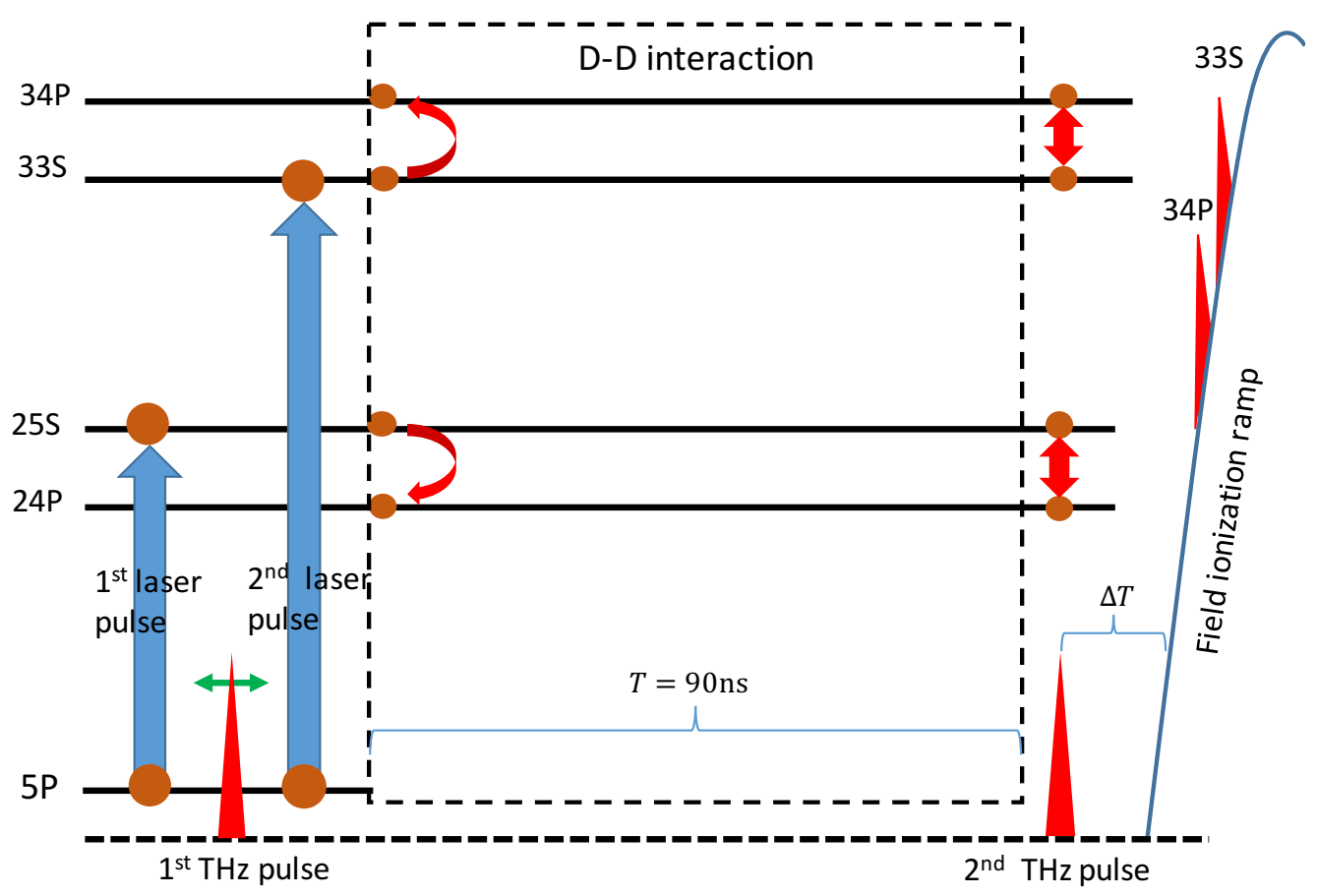

Figure 6.4: Time line for experiment operation. The first laser pulse excites a portion of atoms from $5 p$ to $25 s$, generating atoms $\mathrm{A}$. The first $\mathrm{THz}$ pulse creates a wavepacket composed of $25 \mathrm{~s}, 24 \mathrm{p}$, and $25 \mathrm{p}$ (not shown in the diagram for simplicity) on atoms A and this point could be considered as the $t=0$ point in Equation (6.3). Right after the first THz pulse, a second laser pulse excites another portion of atoms from $5 p$ to $33 s$, creating atoms B. After the creation of atoms B, the DD interaction enables population transfer between $25 \mathrm{~s}$ and $24 \mathrm{p}$, and $33 \mathrm{~s}$ and $34 \mathrm{p}$. The DD interaction lasts for $90 \mathrm{~ns}$, and then a second $\mathrm{THz}$ pulse, working as a probe, redistributes the population in each state. The field ionization ramp is applied as soon as practical after the $2^{\text {nd }} \mathrm{THz}$ pulse to make sure $\Delta T$, which is the time interval between the total shut off of DD interactions and the second THz pulse, is set as close to 0 as possible. The ion signal collected by the MCP reveals the population in each state. A Michelson interferometer, with a variable-length delay-stage in one arm, is used to split each $790 \mathrm{~nm}$ pulse which is to generate $\mathrm{THz}$ radiation pulses, into a pump-probe pair with a delay $\sim 90 \mathrm{~ns}$.

The delay between the two THz pulses is scanned over a short time interval ( $\sim 0.5 \mathrm{~ns})$.

Depending on the temporal separation of the two THz pulses, amplitude transferred from $33 s$ to $34 p$ by the second $\mathrm{THz}$ pulse will interfere constructively or destructively with amplitude transferred to $34 p$ via the DD interaction. This interference produces a modulation in the measured $34 p$ population as a function of relative THz pulse delay [14]. Assuming the $34 p$ population is measured immediately after the $2^{\text {nd }} \mathrm{THz}$ pulse, any delay dependent interference in the $34 p$ yield indicates that coherent wavepacket motion has been 
induced on atoms B. Accordingly, it implies the establishment of correlated electron wavepackets on neighboring atoms (see Equation (6.7)) through controlled DD interactions.

Of course, the experiment is performed with a randomly distributed ensemble in the MOT instead of a single atom pair. So it is necessary to consider the macroscopic dephasing that will result from the variation in DD coupling strength between atom pairs in the MOT. Figure 6.5 shows the rapid dephasing of the wavepackets as computed using a simulation similar to that used to produce Figure 6.2 and Figure 6.3, but including an integration over the random nearest neighbor separations in the MOT ensemble. In order to detect the oscillation of the induced wavepacket, the integral of the interaction strength, $\varepsilon$, over the interaction time, $T$, should be kept less than $\pi / 2$ for the majority of the atoms so that the wavepacket oscillations on these atoms remain in phase. Accordingly, for an ensemble with a density $\sim 2 \times 10^{9} \mathrm{~cm}^{-3}$, the DD interaction time should be kept to less than $400 \mathrm{~ns}$.
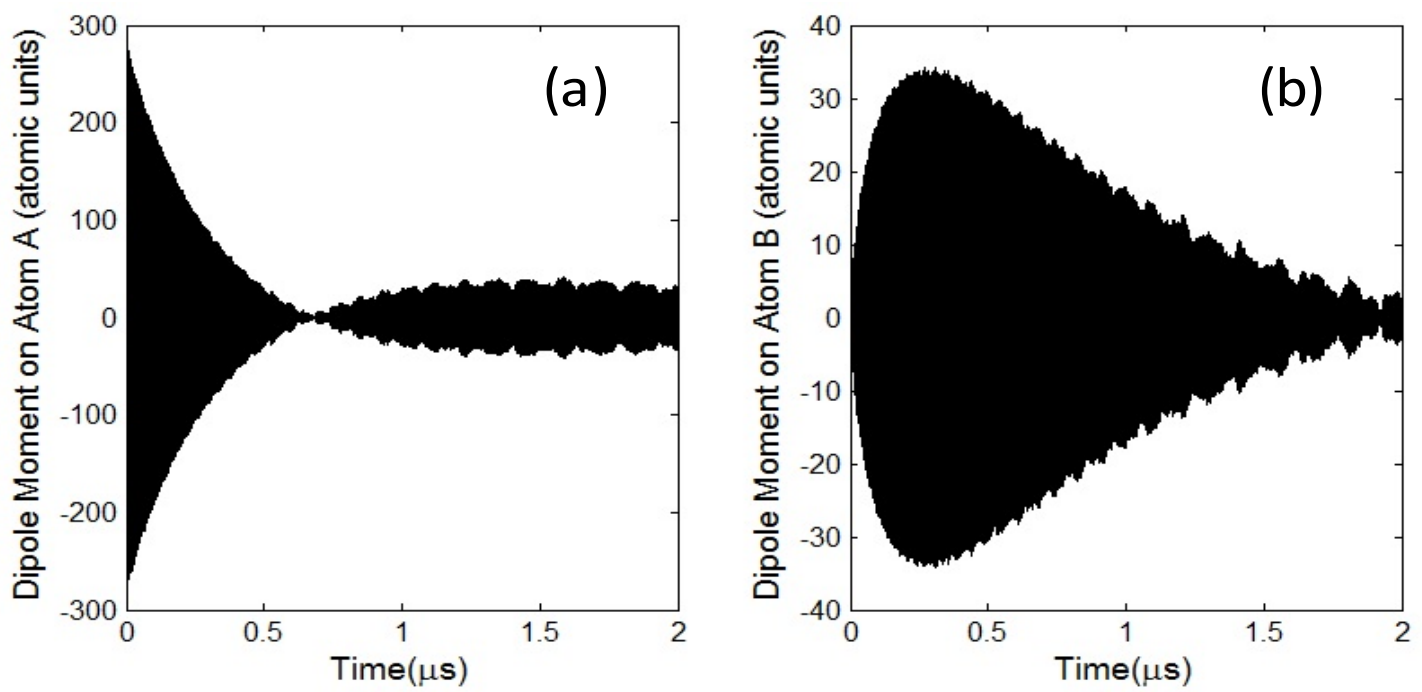

Figure 6.5: Calculated average dipole moment on individual atoms for coupled via DD interactions. The plots are analogous to those shown in Figure 6.2, but a random ensemble. The density of the ensemble is $\sim 2 \times 10^{9} \mathrm{~cm}^{-3}$. Compared to Figure 6.3 , macroscopic coherence is quickly lost because of the dephasing of the wavepackets on different atom pairs.

Beyond decoherence, the other factor affecting the choice of the experimental DD interaction time is the magnitude of the $34 p$ population. If the DD interaction time is too 
short, it is hard to detect the small $34 p$ population, much less the population interference. So when determining the DD interaction time, there is a balance between maintaining wavepacket coherence and generating detectable $34 p$ interference. Figure 6.6 shows the $34 p$ population generated via the resonant energy transfer as a function of the DD interaction time in the experiment.

Considering the wavepacket dephasing, the range of Rydberg densities that can be readily produced in the MOT, the dependence of the $34 p$ signal on interaction time, and the difficulty producing an optical delay line with a maximum pulse separation $>100 \mathrm{~ns}$, we determine an optimum density $\sim 2 \times 10^{9} \mathrm{~cm}^{-3}$ and interaction time $T \approx 90 \mathrm{~ns}$ for the experiment.

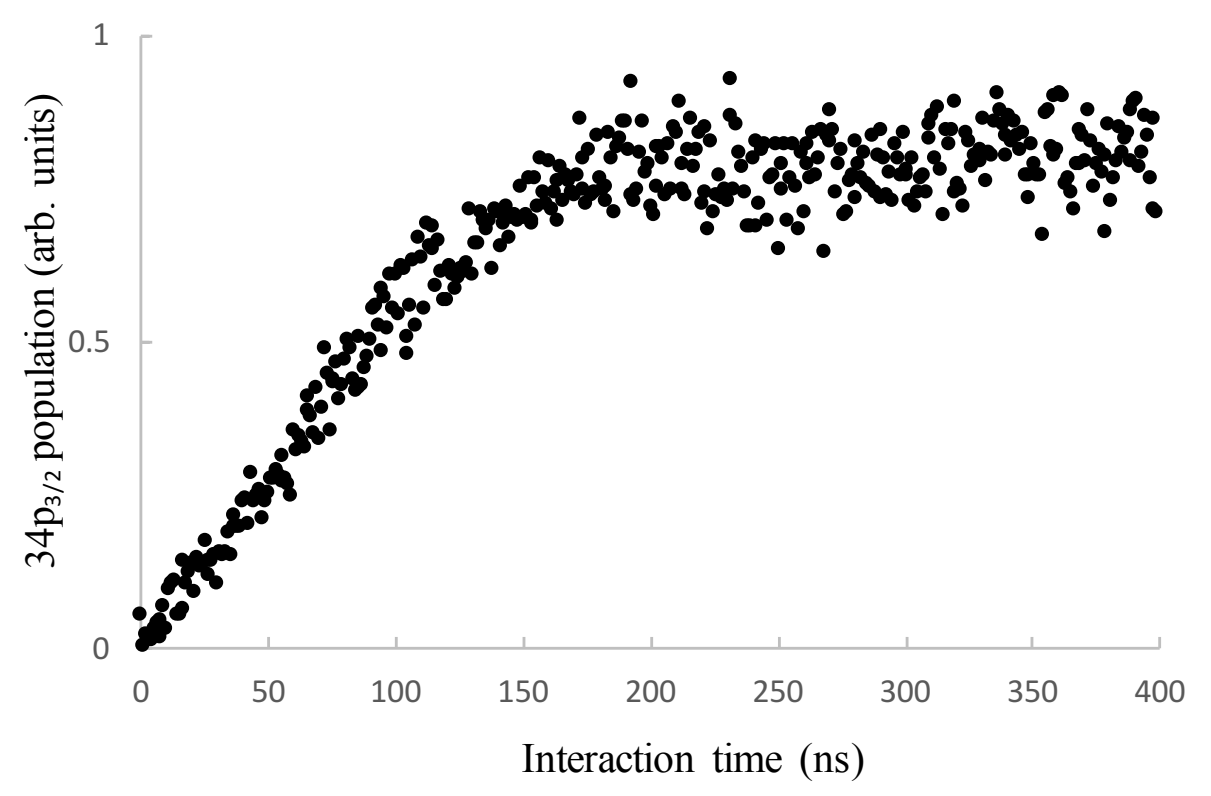

Figure 6.6: Measured population transfer to $34 p$ as a function of interaction time $T$ for a density $\sim 2 \times 10^{9} \mathrm{~cm}^{-3}$. The initial state is $(|25 s\rangle+|24 p\rangle)|33 s\rangle$. After $T \sim 200 \mathrm{ns,}$ the transfer is saturated, due to the dephasing of the DD mediated population transfer process.

After setting the interaction time $T$, we measure the $34 p$ population as the relative $\mathrm{THz}$ delay is scanned by $\sim 0.5 \mathrm{~ns}$. To detect the $34 p$ atoms, we apply a field ionization (FI) ramp 
as soon as possible following the $2^{\text {nd }} \mathrm{THz}$ pulse. The resulting ions are collected by the MCP.

Figure 6.7 shows the population interference when scanning the $\mathrm{THz}$ delay. It appears to suggest a coherent wavepacket motion on atoms B. However, additional spectral analysis of the signal (Figure 6.8) shows that there is another, less interesting, effect that is at least partially responsible for the population interference.

If the signal modulations were produced solely by an interference between the $34 p$ amplitude produced by the resonant $\mathrm{DD}$ interaction and that excited by the second $\mathrm{THz}$ pulse, then there should be only one peak (matching the $33 s \rightarrow 34 p$ transition at 11.4 $\mathrm{cm}^{-1}$ ) in the spectrum obtained by fast Fourier transforming (FFT) the delay-dependent $34 p$ signal. However, the FFT analysis clearly shows two additional peaks. The feature at $9.9 \mathrm{~cm}^{-1}$ corresponds to the energy splitting between the $25 s$ and $25 p_{3 / 2}$ states. The peak at $11.1 \mathrm{~cm}^{-1}$ matches the $25 \mathrm{~s}$ and $24 p_{3 / 2}$ transition energy. Also, it is important to remember that the feature at $11.4 \mathrm{~cm}^{-1}$ also matches the $25 s \rightarrow 24 p_{1 / 2}$ energy interval. Apparently, all of the spectral peaks observed in the $34 p$ population signal might be due to coherence involving the $25 s$ state. 
For comparison, Figure 6.9 shows the time dependent population in the $25 \mathrm{~s}$ state as a function of delay between the two THz pulses and Figure 6.10 shows its Fourier transform. As with the $34 p$ signal, the second THz pulse probes a previously created coherence. In this case, that coherence is associated with the $25 s+24 p$ wavepacket produced by the first $\mathrm{THz}$ pulse. Although taken under slightly different $\mathrm{THz}$ conditions in which the pulse spectrum was not as well optimized for preferential excitation of $33 s \rightarrow 34 p$, the same spectral features that appear in the $34 p$ population are clearly observed in the $25 \mathrm{~s}$ population.

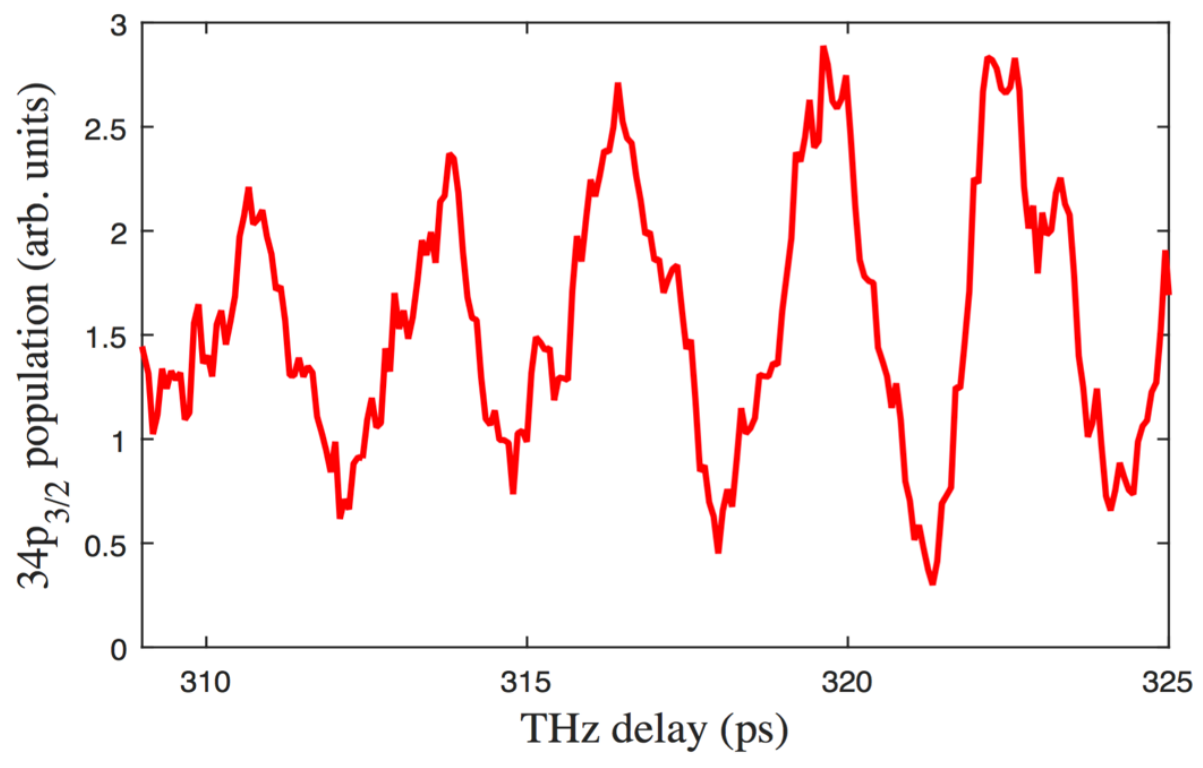

Figure 6.7: $34 p$ population over a short range of THz delays. Shown is the averaged result from 20 measurements. The main period of the oscillation is $\sim 2.9 \mathrm{~ns}$. The yvalues do not accurately represent the modulation amplitude to DC background. In experiment, the oscillation amplitude is about $1 / 4$ of the DC background. 


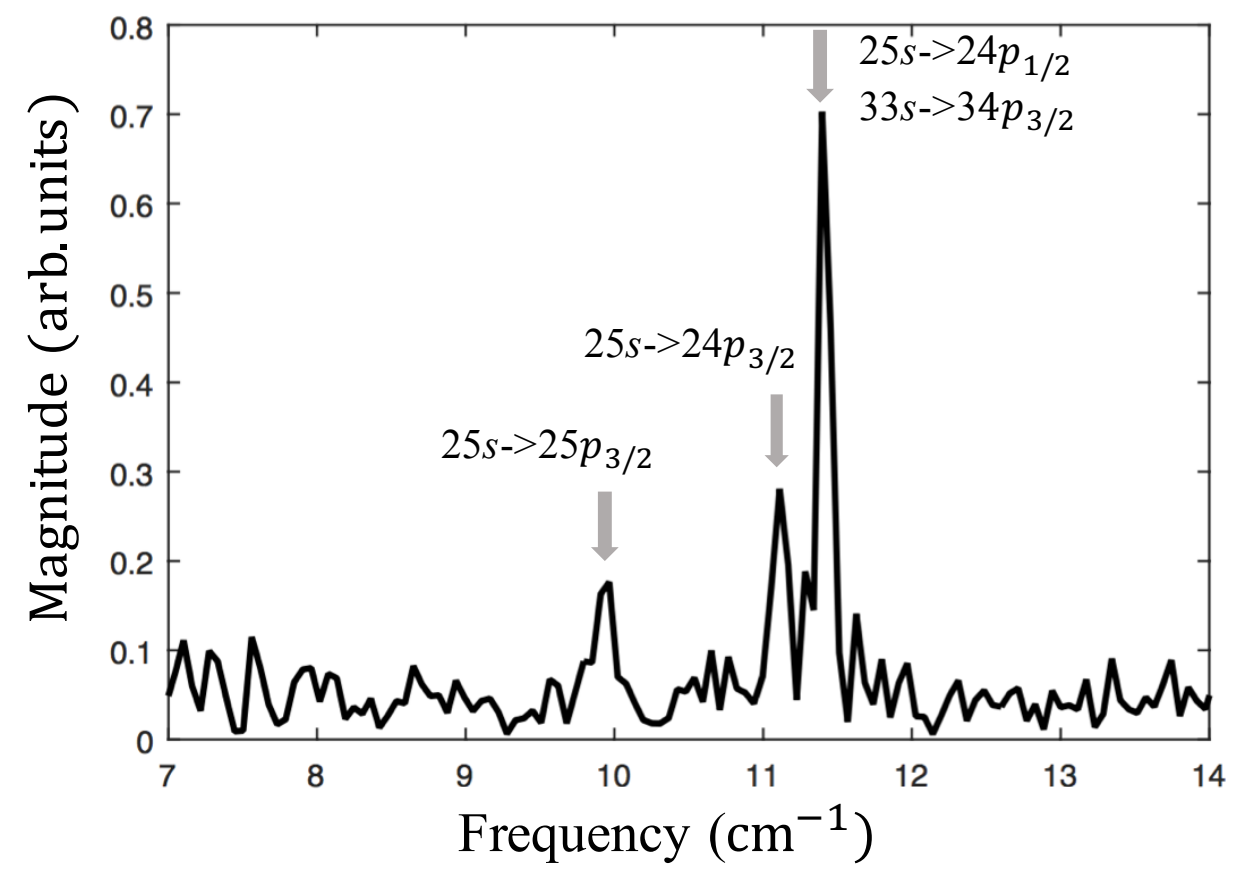

Figure 6.8: FFT of the delay-dependent $34 p$ population obtained with a short delay $\Delta T \cong 10 \mathrm{~ns}$, between the $2^{\text {nd }} \mathrm{THz}$ pulse and the FI ramp. The three peaks shown in the plot (from left to right) are $9.9 \mathrm{~cm}^{-1}\left(25 s_{-}>25 p_{3 / 2}\right), 11.1 \mathrm{~cm}^{-1}\left(25 s^{->}-24 p_{3 / 2}\right)$, and $11.4 \mathrm{~cm}^{-1}\left(25 s^{->} 24 p_{1 / 2}\right.$ and $\left.33 s->34 p_{3 / 2}\right)$, respectively.

Due to the wavepacket motion initiated by the first $\mathrm{THz}$ pulse, the modulations in the $25 s$ population produced by the second $\mathrm{THz}$ pulse are large and have fairly high contrast. At the same time, due to the small population transfer to $34 p$, the population in $33 \mathrm{~s}$ is relatively unchanged by the DD interaction between the two THz pulses. Any population transfer to $34 p$ via the resonant DD coupling after the $2^{\text {nd }} \mathrm{THz}$ pulse is directly proportional to the populations in $25 s$ and $33 s$ during that time interval. As a result, if the DD interaction is allowed to continue for a time, $\Delta T$, after the second $\mathrm{THz}$ pulse, the $34 p$ population that is produced via the $25 s 33 s \leftrightarrow 24 p 34 p$ coupling during that time will be modulated with the same phase and frequency as the $25 \mathrm{~s}$ population. Unfortunately, we cannot instantaneously shut off the DD coupling after the second $\mathrm{THz}$ pulse due to the small $(<10 \mathrm{~ns})$ uncertainty in precisely when the atoms are exposed to the $\mathrm{THz}$ and $\mathrm{FI}$ pulses and the time during the rise ( $\sim 100 \mathrm{~ns}$ to reach its half maximum) of FI pulse at which the atoms are decoupled and 
can no longer interact. Thus, the $34 p$ signal is always "contaminated" by this imperfect measurement artifact which can actually dominate the delay dependence in the $34 p$ signal.

Fortunately, the $11.4 \mathrm{~cm}^{-1}$ oscillation which is associated with the DD coherence transfer which we are attempting to observe is also predicted to be $90^{\circ}$ out of phase with the $25 s$ modulation artifact at the same frequency (see Figure 6.3). Accordingly, a non-zero phase shift of the $11.4 \mathrm{~cm}^{-1}$ oscillation in $34 p$, relative to those in $25 \mathrm{~s}$, is a signature of the development of wavepacket motion on atoms B due to the electron dynamics on atoms A and the correlations between the two sets of atoms. As shown in Figure 6.11, the degree of the phase-shift is expected to depend on the magnitude of the signal contribution from the coherence transfer relative to that due to the artifact.

Conveniently, the oscillations in the $25 s$ and $34 p$ signals at the additional $25 s \rightarrow n p$ transition energies, which are unrelated to the coherence transfer, should be perfectly inphase. Thus, their relative phases can be (and are) used as an independent check of the quality of individual data runs and our ability to accurately retrieve the relative phase of interest at $11.4 \mathrm{~cm}^{-1}$.

For experimental convenience, in the actual data collection runs, we do not compare the phases of the modulations in the $25 s$ and $34 p$ populations as this would require that we collect consecutive data sets with significantly different FI ramp amplitudes. Instead, we compare the $34 p$ signal acquired with a minimal delay, $\Delta T \cong 10 \mathrm{~ns}$ between the second $\mathrm{THz}$ pulse and the FI ramp with that obtained with a much longer $\Delta T \cong 600 \mathrm{~ns}$ delay. For the long delay, the population transfer to $34 p$ is completely dominated by the interaction time after the $2^{\text {nd }}$ THz pulse. Thus, for all frequencies, the strong $34 p$ modulations observed with the long delay are purely due to the artifact (see Figure 6.12) and should be in phase with the corresponding modulations in the $25 \mathrm{~s}$ signal. We have confirmed experimentally that, for $\Delta T \cong 600 \mathrm{~ns}$, the $34 p$ and $25 s$ modulations are indeed in-phase $\left(0 \pm 3^{0}\right)$. 


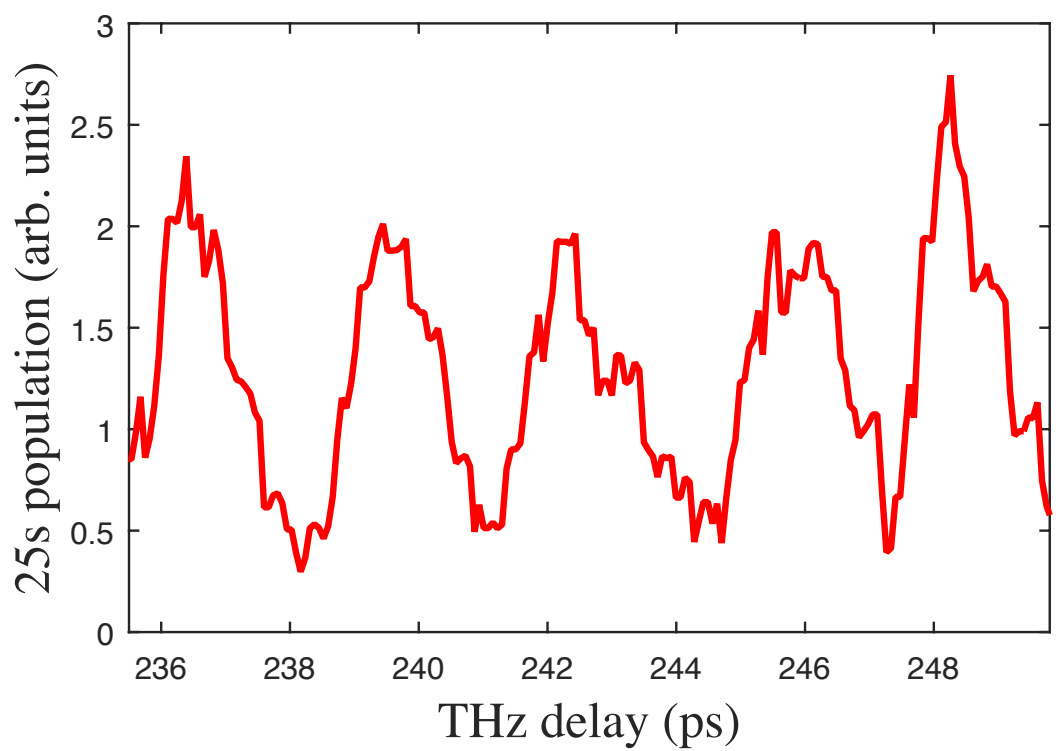

Figure 6.9: Measured 25s population over a short range of THz delays.

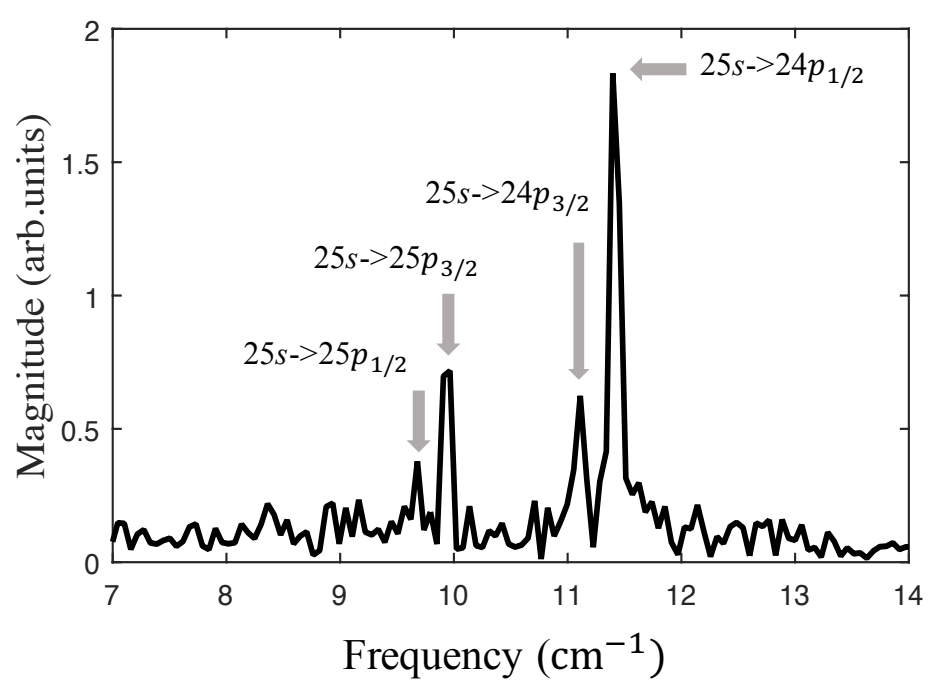

Figure 6.10: FFT of the delay-dependent $25 s$ population. The four peaks shown in the plot (from left to right) are $9.7 \mathrm{~cm}^{-1}\left(25 s->25 p_{1 / 2}\right), 9.9 \mathrm{~cm}^{-1}\left(25 s^{-}>25 p_{3 / 2}\right)$, $11.1 \mathrm{~cm}^{-1}\left(25 s^{-}>24 p_{3 / 2}\right)$, and $11.4 \mathrm{~cm}^{-1}\left(25 s^{->}-24 p_{1 / 2}\right)$ respectively. 


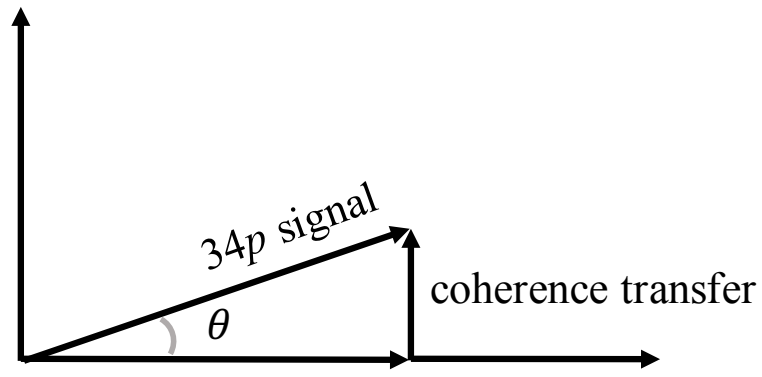

artifact

Figure 6.11: Phasor diagram illustrating the contributions to the detected $34 p$ signal modulations as well as the phase difference $\theta$ between the measured oscillations in $34 p$ and $25 s$. If the coherence transfer contribution is very small compared to the delayed detection associated with the $25 s$ population modulation, then the presence of coherence transfer has little effect on the magnitude of the $11.4 \mathrm{~cm}^{-1}$ oscillation in the $34 p$ signal. However, the presence of the coherence transfer can be detected through the phase shift, $\theta$.

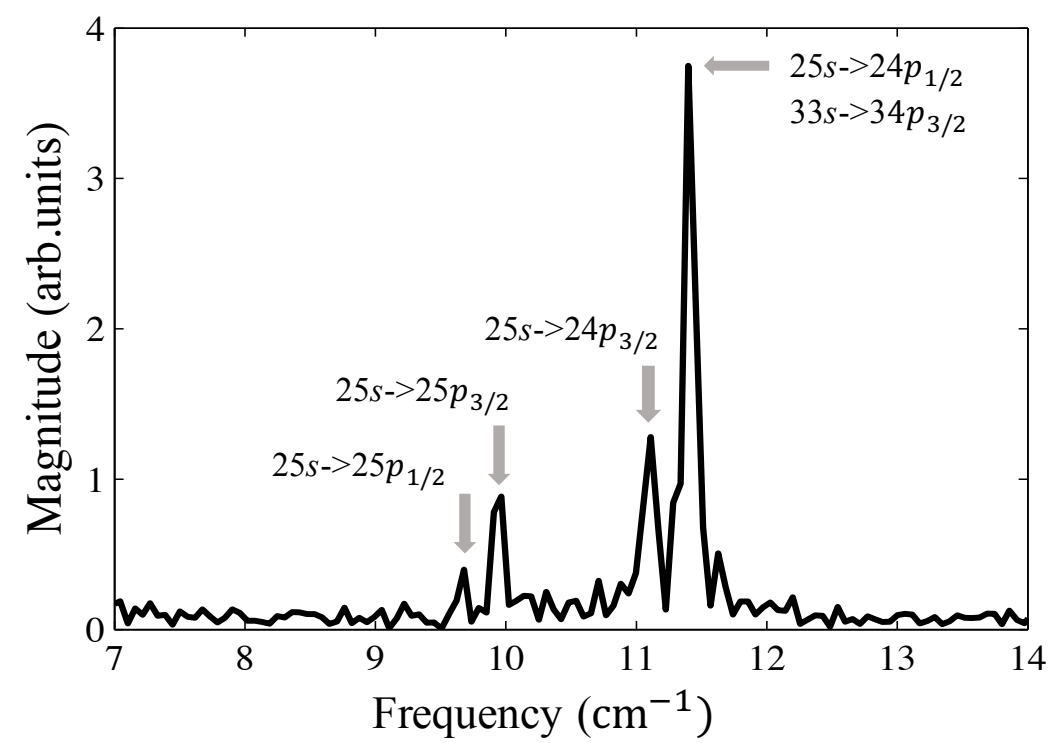

Figure 6.12: FFT of the time-dependent $34 p$ population at large $\Delta T$ (>600 ns). Peaks associated with various coherences are labeled.

In the experiment, a THz delay scan at a small $\Delta T(\sim 10 \mathrm{~ns})$ and a large $\Delta T(\sim 600 \mathrm{~ns})$ are performed. The two data sets compose a "pair scan". About 30 total pair scans were collected over a total of 6 different days. Ten of these data pairs were rejected due to the large phase differences $\left(>36^{0}\right)$ between the reference oscillations at $9.9 \mathrm{~cm}^{-1}$ or 11.1 $\mathrm{cm}^{-1}$. The other 20 pair scans have small phase differences $\left(<18^{0}\right)$ at these reference frequencies. Note that the magnitude of the $11.9 \mathrm{~cm}^{-1}$ frequency component is 
considerably larger than that of the references. Thus, the uncertainty in the $11.4 \mathrm{~cm}^{-1}$ phase is substantially small. Each of these pairs shows a positive phase advance of the $34 p$ population modulation at small $\Delta T$ relative to that at large $\Delta T$. The average phase shift obtained from the 20 individual pair scans is $21^{0} \pm 9^{0}$.

Figure 6.13 shows the $34 \mathrm{p}$ population variations, for small and large $\Delta T$, as a function of the relative THz delay. The two curves are obtained by averaging the 20 data pairs used to determine the relative phase shift. The time axis for the different pairs were shifted to account for temporal drifts between scans taken at very different times. With the shifts, we define a phase of $0^{0}$ for the $11.4 \mathrm{~cm}^{-1}$ peak in the long delay scans, but preserve the relative phase shift between long and short delay scans in the each pair. The phase advance in the short delay scans $\left(\sim 20^{\circ}\right)$ is clearly visible, providing strong evidence for the predicted wavepacket coherence transfer between atoms.

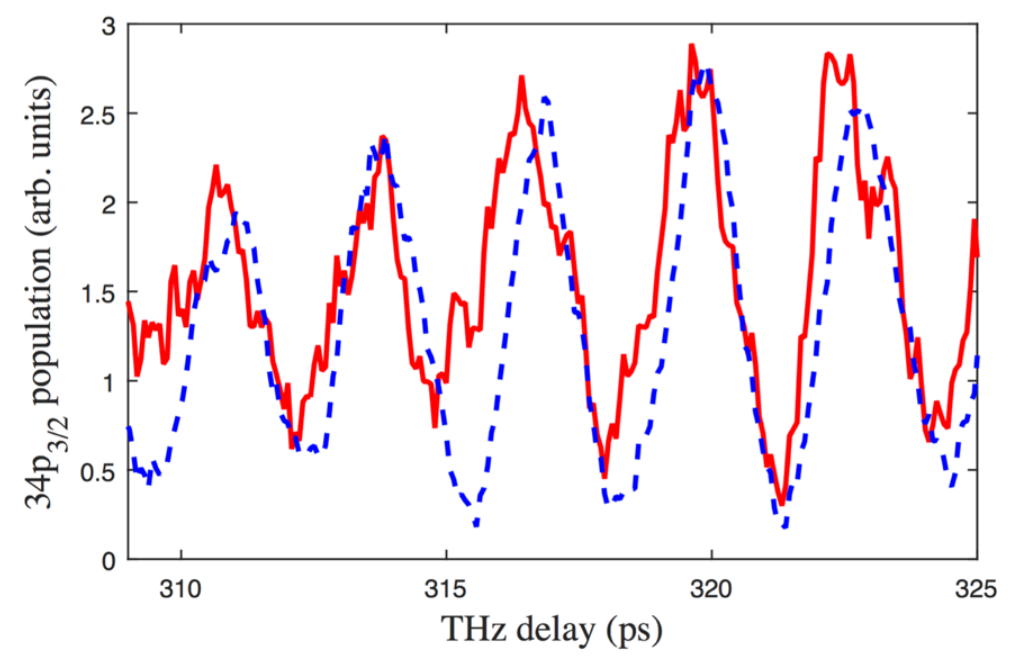

Figure 6.13: Averaged $34 p$ population variation as a function of relative $\mathrm{THz}$ delays for short $(\Delta T \sim 10 \mathrm{~ns}$, red solid) and long $(\Delta T \sim 600 \mathrm{~ns}$, blue dashed) ionization delays. The signal variation at long delays $\Delta T$ has been reduced by a factor of 3 to make a better comparison with the interference at small $\Delta T$. The signal variation at large and small $\Delta T$ are about $1 / 4$ of their DC backgrounds, repectively. 


\subsection{Analysis and Discussion}

A simulation has been performed to examine if the measured $21^{0}$ phase difference is reasonable. The simulation calculates the phase difference between the modulations in the $34 p$ and 25 s populations as a function of the detection delay $\Delta T$, while keeping the DD interaction time, $T \cong 90 \mathrm{~ns}$ a constant. Results are shown in Figure 6.14.

While every attempt is made to capture the essential features of the experiment, the simulation is only approximate. First, the range of states is limited. Only the following states are included: $25 \mathrm{~s}, 24 p_{1 / 2}, 24 p_{3 / 2}, 25 p_{1 / 2}, 25 p_{3 / 2}, 33 \mathrm{~s}, 33 p_{1 / 2}, 33 p_{3 / 2}, 34 p_{1 / 2}, 34 p_{3 / 2}$. Second, atom motion and beyond nearest neighbor interactions are completely neglected. Third, the time-dependence of the THz pulses and their amplitudes are not precisely known. However, different combinations of pulse parameters have been used to ensure that the population redistribution by the $\mathrm{THz}$ pulses is as close as possible to that measured. In the experiment, we estimate the amplitude of the $2^{\text {nd }} \mathrm{THz}$ pulse to be approximately half of that of the $1^{\text {st }} \mathrm{THz}$ pulse.

The predicted $90^{\circ}$ phase shift in the $34 \mathrm{p}$ and $25 \mathrm{~s}$ population is based on a perturbation model of the THz-atom interaction. As shown in Figure 6.14, different amplitudes of the $2^{\text {nd }} \mathrm{THz}$ pulse result in different phase differences. As expected, the phase difference declines rapidly with increasing $\Delta T$.

Another source of uncertainty comes from the Rydberg density measurement. Previous measurements of resonant energy transfer [11][15] indicate that the density measurement is accurate to approximately $50 \%$. If the actual density in the experiment is higher than we estimate, the DD interactions would be stronger, accelerating the decrease in phase difference as a function of $\Delta T$. 
While the simulation does not perfectly reproduce the experimental observations, it qualitatively supports our principal findings. As shown in Figure 6.14, the phase difference between the $34 p$ and $25 s$ interferences is indeed positive and smaller than $90^{\circ}$. Moreover, the phase shift rapidly approaches 0 with increasing $\Delta T$.

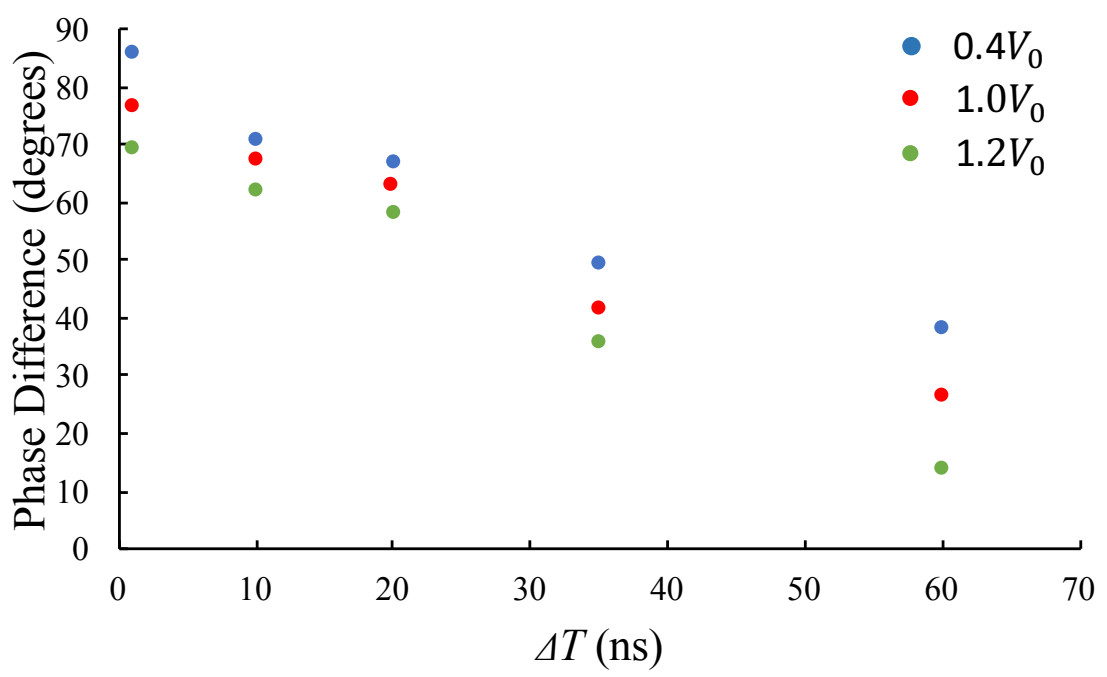

Figure 6.14: Calculated phase difference between the $34 p$ and $25 s$ population oscillations as a function of $\Delta T$ for different amplitudes of the $2^{\text {nd }} \mathrm{THz}$ pulse. The density of the ensemble is $\sim 2 \times 10^{9} \mathrm{~cm}^{-3}$. $V_{0}$ is the field amplitude of the $1^{\text {st }} \mathrm{THz}$ pulse, which is chosen to be $50 \mathrm{~V} / \mathrm{cm}$ to match the $25 \mathrm{~s} \rightarrow 24 \mathrm{p}$ population transfer observed in the experiment.

\subsection{Conclusion}

We have explored the mechanism of wavepacket coherence transfer from one Rydberg atom to a neighboring Rydberg atom via DD interactions utilizing the resonant DD coupling transition $25 s 33 s \leftrightarrow 24 p 34 p$. The phase-shift of the observed interference modulations in the $34 p$ signal, relative to that in $25 s$, is a signature of wavepacket coherence transfer between atoms driven by electron correlations resulting from the controlled DD coupling between them.

Future experiments may take advantage of ensembles having more well-defined atom separations to observe the complete transfer of wavepacket motion, back and forth, between initial and induced wavepackets. 


\section{Bibliography}

[1] Levis, R. J., and H. A. Rabitz. J. Phys. Chem. A, 106, 6427 (2002).

[2] Dantus, Marcos, and Vadim V. Lozovoy. Chem. Reviews 104, 1813 (2004).

[3] Khaneja, Navin, Timo Reiss, Cindie Kehlet, Thomas Schulte-Herbrüggen, and Steffen J. Glaser. Journal of Magnetic Resonance 172, 296 (2005).

[4] Matthieu Viteau, Amodsen Chotia, Maria Allegrini, Nadia Bouloufa, Olivier Dulieu, Daniel Comparat, and Pierre Pillet, Science 321, 232 (2008).

[5] Lien, Chien-Yu, Christopher M. Seck, Yen-Wei Lin, Jason HV Nguyen, David A. Tabor, and Brian C. Odom. Nature communications 5, 4783 (2014).

[6] J. J. García-Ripoll, P. Zoller, and J. I. Cirac. Phys. Rev. Lett. 91, 157901 (2003).

[7] Scharfenberger, Burkhard, William J. Munro, and Kae Nemoto. New Journal of Physics 16, 93043 (2014).

[8] Steve Winder, Joseph Carr, "Newens Radio and RF Engineering Pocket Book", $3^{\text {rd }}$ edition, Newens (2002).

[9] Beverley. R. Green, Jan. M. Andersion, W.W. Parson, "Light-Harvesting Antennas in Photosynthesis", Springer Netherlands (2003).

[10] R.R. Jones and L. D. Noordam, Advances in Atomic Molecular and Optical Physics 38, 1 (1998).

[11] Mary Kutteruf, Coherence in Rydberg Atoms: Measurement and Control, $\mathrm{PhD}$ thesis, University of Virginia (2010).

[12] W.R. Anderson, J.R. Veale, and T.F. Gallagher, Phys. Rev. Lett. 80, 249 (1998).

[13] R. Anderson, M.P.Robinson. J.D.D Martin, and T.F. Gallagher, Phys. Rev. A. 65, 063404 (2002).

[14] L.M.Duan, G.G.Guo, Phys. Rev. Lett. 79, 1953 (1997). 
[15] B. G. Richards, R. R. Jones, Physical Review A 93, 042505 (2016). 


\section{Summary and Future Directions}

This chapter summarizes the results of the projects described in this dissertation and provides some thoughts on possible extensions for future study. 
CHATPTER 7 SUMMARY AND FURTURE DIRECTIONS

Electron dynamics in DD coupled Rydberg atoms show some unique properties that do not exist in individual atoms. The goal of the research in this dissertation is to reveal and control the electron dynamics influenced by DD interaction in cold Rydberg gases. Our results have clarified and provides further insight into, fundamental few body physics problems in atomic physics, and could potentially benefit practical applications involving quantum control of few- and many-body systems, from quantum simulation and information processing, to the quantum control of energy transport in photosynthetic systems.

The project in Chapter 4 examines the decay of $n s$ and $n p$ Rydberg states with high principal quantum numbers (from 26 to 40 ) in a cold $\mathrm{Rb}$ gas. The measured time dependence of the Rydberg population is well described by numerical simulations which consider only spontaneous emission and population transfer by blackbody radiation. No evidence for the previously reported collective decay is found at atom densities up to $3 \times$ $10^{9} \mathrm{~cm}^{-3}$. Our conclusion is that the suppression of collective emission is likely due to variations in transition energies within the atom sample, dominated by inhomogeneities in dipole-dipole exchange interactions for initial $s$ states, or by a combination of DD and electric field inhomogeneities for the initial $p$ states. We propose that DD exchange process will dephase superradiance unless the geometric enhancement factor $L / \lambda \gg 1$. An interesting future experiment would be to measure the lifetimes of lower $n$-states at constant sample excitation length, $L$ and observe a transition from predominantly single atom to superradiance decay as $L / \lambda$ increases from $\langle 1$ to $\gg 1$.

The project in Chapter 5 characterizes the role of DD interactions in the evolution of Rydberg wavepackets. As the wavepackets evolve they are influenced by DD interactions, predominantly pairwise excitation-exchange processes of the form $|s\rangle|p\rangle \leftrightarrow|p\rangle|s\rangle$. The coherent electronic evolution of the ensemble dephases due to the variation in DD coupling 
strength between atom pairs in the MOT. An extension of this experiment would be to measure the macroscopic coherence in an ensemble which has better controlled separations between atoms than a random ensemble. The expectation is that the macroscopic coherence would be maintained in a separation controlled ensemble than a random ensemble, since the variation in DD coupling strength is smaller. Indeed, long term macroscopic coherence could serve as an indicator of uniform atom separation in the MOT. Brian Richards is currently attempting to utilize controlled DD interactions to manipulate the position correlation function of cold trapped atoms. If his project is successful, his technique would enable this measurement.

The project in Chapter 6 utilizes the resonant energy transfer $25 s 33 s \leftrightarrow 24 p 34 p$ to demonstrate that electron correlations, induced by controlled DD interactions, can enable the coherent transfer of electronic wavepacket motion from atoms to their neighbors. The mixed-parity wavepacket composed of $25 s$ and $24 p$ on one set of atoms induces, via DD interactions, wavepacket generation on another set of atoms, which are initially on state $33 s$. The coherence transfer is observed by measuring the phase difference between the initial and induced wavepacket oscillations. A future experiment based on this project might systematically investigate the disappearance of coherence transfer signal due to the varying separation between atoms in the ensemble. As the atom density or the interaction time (as mentioned in Chapter 6) increases, the variation in the induced wavepackets phase over the MOT should preclude detection of the wavepacket oscillations. Alternatively, if the atom separation can be unified, complete transfer of wavepacket motion, back and forth, between initial and induced wavepackets might be observed. This would also open up the possibility for exploring beyond nearest neighbor effects in the wavepacket transfer. 